\title{
LIMIT GROUP INVARIANTS FOR NON-FREE CANTOR ACTIONS
}

\author{
STEVEN HURDER AND OLGA LUKINA
}

\begin{abstract}
A Cantor action is a minimal equicontinuous action of a countably generated group $\mathrm{G}$ on a Cantor space X. Such actions are also called generalized odometers in the literature. In this work, we introduce two new conjugacy invariants for Cantor actions, the stabilizer limit group and the centralizer limit group. An action is wild if the stabilizer limit group is an increasing sequence of stabilizer groups without bound, and otherwise is said to be stable if this group chain is bounded. For Cantor actions by a finitely generated group G, we prove that stable actions satisfy a rigidity principle, and furthermore show that the wild property is an invariant of the continuous orbit equivalence class of the action.

A Cantor action is said to be dynamically wild if it is wild, and the centralizer limit group is a proper subgroup of the stabilizer limit group. This property is also a conjugacy invariant, and we show that a Cantor action with a non-Hausdorff element must be dynamically wild. We then give examples of wild Cantor actions with non-Hausdorff elements, using recursive methods from Geometric Group Theory to define actions on the boundaries of trees.
\end{abstract}

\section{INTRODUCTION}

In this paper, we investigate the structure of non-free Cantor group actions, and the relationship between the dynamics of the action and the algebraic properties of the group. One of our main results is the definition of direct limit groups which are conjugacy invariants of the action, and we investigate the relation between these new invariants and the dynamics of the action. Our results are illustrated by examples of Cantor actions in the literature, and also those constructed in Section 8 .

We assume as standing hypotheses that $G$ is a countably infinite group, $\mathfrak{X}$ is a Cantor space, and $\Phi: G \rightarrow \operatorname{Homeo}(\mathfrak{X})$ is an action of $G$. We sometimes assume in addition that $G$ is finitely generated, and when required this hypothesis will be indicated. We denote the action by $(\mathfrak{X}, G, \Phi)$, and write $g \cdot x$ for $\Phi(g)(x)$ when the action is clear.

The action is minimal if for all $x \in \mathfrak{X}$, its orbit $\mathcal{O}(x)=\{g \cdot x \mid g \in G\}$ is dense in $\mathfrak{X}$. Let $d_{\mathfrak{X}}$ be a metric on $\mathfrak{X}$ compatible with the topology. The action $(\mathfrak{X}, G, \Phi)$ is equicontinuous with respect to $d_{\mathfrak{X}}$ if for all $\varepsilon>0$ there exists $\delta>0$, such that for all $x, y \in \mathfrak{X}$ and $g \in G, d_{X}(x, y)<\delta$ implies that $d_{X}(\Phi(g) \cdot x, \Phi(g) \cdot y)<\varepsilon$. This property is independent of the choice of the metric $d_{\mathfrak{X}}$ on $\mathfrak{X}$.

In this paper, a Cantor action $(\mathfrak{X}, G, \Phi)$ is assumed to be minimal and equicontinuous. We also occasionally discuss actions which are not assumed to be minimal or equicontinuous, and these will be called general Cantor actions.

Cantor actions can be divided into two types, stable and wild. For example, a free Cantor action of an abelian group $G$ is stable. The stable property is a weaker form of the more well-known "topologically free" property assumed in many works on the study of Cantor actions. Topologically free general Cantor actions have been extensively analyzed in the literature [7, 13, 38, 49, and the authors' work in [19, 36] extends some of these results to stable Cantor actions. The distinction between stable and topologically free actions is discussed in Section 6 On the other hand, the structure and properties of wild Cantor actions are less well-known, and in this work we study this class of actions in depth, obtaining new invariants and new classification results.

OL is supported by FWF Project P31950-N35.

2010 Mathematics Subject Classification. Primary: 37B45, 37C15, 37C85; Secondary: 57S10.

Keywords: minimal Cantor actions, continuous orbit equivalence, rigidity, non-Hausdorff groupoids.

Version date: April 25, 2019; revised January 9, 2020. 
The first example of a wild Cantor action was constructed by Schori in [51], as the monodromy action for a weak solenoid which is not homogeneous. The "Schori solenoid" gave a counter-example to a question raised by McCord in [42, and became the focus of further study. The analysis of this example in the authors' work with Jessica Dyer [19] introduced the notion of the wild property for a minimal equicontinuous action of a group $G$ on a Cantor space $\mathfrak{X}$. It was observed by the authors in 19 that the Molino Theory for foliated spaces developed in [2] applies for weak solenoids whose monodromy actions are stable, but fails for weak solenoids whose monodromy actions are wild.

The work [35 also gave a general method for constructing wild Cantor actions, and used the wild property to show that these examples yield uncountably many classes of non-homeomorphic, nonhomogeneous weak solenoids. This work suggested that a classification of weak solenoids up to homeomorphism requires a better understanding of the class of wild Cantor actions.

In the subsequent work [36], the authors studied the classification problem for stable Cantor actions, and gave a generalization of the rigidity theorems for Cantor actions of Cortez and Medynets [13] and $\mathrm{Li}[38$. We showed that for stable Cantor actions, continuous orbit equivalence implies return equivalence of the actions. Our work also showed that if $G$ is a finitely generated nilpotent group, then every Cantor action by $G$ is stable. Thus, for Cantor actions by finitely generated nilpotent groups, one has a direct approach to their classification via the rigidity principle.

The study of stable Cantor actions also highlighted the role of non-Hausdorff elements for the action, as discussed in Section 7.2 and Definition 7.3. An action with a non-Hausdorff element is at the opposite extreme of a stable action. Another theme of our work is to investigate wild actions with non-Hausdorff elements.

We now discuss the main results of this work. Sections 2 and 3 give the basic background material on Cantor actions that we require. This material overlaps with discussions and results in the authors' previous papers [17, 35, 36], and somewhat also with the works [13, 38, but is discussed here in sufficient detail as necessary for the remainder of the paper.

Section 2 describes a model for a Cantor action $(\mathfrak{X}, G, \Phi)$ as a group action on a Cantor homogeneous space, $\mathfrak{X} \cong \mathfrak{G}(\Phi) / \mathfrak{G}(\Phi)_{x}$, where $\mathfrak{G}(\Phi) \subset \operatorname{Homeo}(\mathfrak{X})$ is a profinite group acting transitively on $\mathfrak{X}$, and $\mathfrak{G}(\Phi)_{x} \subset \mathfrak{G}(\Phi)$ is the isotropy subgroup of a point $x \in \mathfrak{X}$. The philosophy of our approach in this paper, is that the study of the "adjoint action" of the isotropy group $\mathfrak{G}(\Phi)_{x}$ on $\mathfrak{G}(\Phi)$ yields conjugacy invariants for the action.

Section 3 discusses the odometer model for a Cantor action, and introduces a number of concepts about these actions which are key to subsequent sections. In particular, the stabilizer group chain of an odometer is defined in (22), and in Section 3.4 the the centralizer group chain is defined.

Section 4 recalls the formal construction of direct limit groups, their equivalence, and properties of these groups. Then Definition 4.14 and Theorem 4.15 combined yield:

THEOREM 1.1. Let $(\mathfrak{X}, G, \Phi)$ be a Cantor action. There are well-defined direct limit groups

(1) the stabilizer limit group $\Upsilon_{s}(\Phi)$,

(2) the centralizer limit group $\Upsilon_{c}(\Phi)$,

defined as the equivalence classes of the stabilizer and centralizer group chains associated to an odometer model for the action. Both $\Upsilon_{s}(\Phi)$ and $\Upsilon_{c}(\Phi)$ are conjugacy class invariants of the action, and there is an inclusion of direct limit groups $\Upsilon_{c}(\Phi) \subset \Upsilon_{s}(\Phi)$.

For a Cantor action of an abelian group $G=\mathbb{Z}^{d}$, both of the limit group invariants in Theorem 1.1 are trivial, and the action is stable. However, for Cantor actions of more general groups $G$, the stabilizer and centralizer limit group invariants can be highly non-trivial. One theme of this work is to use these limit group invariants to obtain a finer classification of non-free Cantor actions, into the following subtypes of actions. 
DEFINITION 1.2 (Definition 4.18). A Cantor action $(\mathfrak{X}, G, \Phi)$ is said to be:

(1) stable if the stabilizer group $\Upsilon_{s}(\Phi)$ is bounded, and wild otherwise;

(2) algebraically stable if the its centralizer group $\Upsilon_{c}(\Phi)$ is bounded, and algebraically wild otherwise;

(3) wild of finite type if the stabilizer group $\Upsilon_{s}(\Phi)$ is unbounded, and represented by a chain of finite groups;

(4) wild of flat type if the stabilizer group $\Upsilon_{s}(\Phi)$ is unbounded, and $\Upsilon_{c}(\Phi)=\Upsilon_{s}(\Phi)$;

(5) dynamically wild if the stabilizer group $\Upsilon_{s}(\Phi)$ is unbounded, and is not of flat type.

All of the possibilities in this definition can be realized by examples of Cantor actions.

Section $[5$ discusses the notion of a locally quasi-analytic Cantor action $(\mathfrak{X}, G, \Phi)$, and the relation between this notion and the more usual notion of a topologically free action. The main result gives an interpretation of the stable property in terms of the "analytic properties" of the adjoint action of the isotropy group $\mathfrak{G}(\Phi)_{x} \subset \mathfrak{G}(\Phi)$ for a homogeneous model for $\mathfrak{X} \cong \mathfrak{G}(\Phi) / \mathfrak{G}(\Phi)_{x}$.

THEOREM 1.3 (Theorem [5.3). Let $(\mathfrak{X}, G, \Phi)$ be a Cantor action, then $\Phi$ is stable if and only if the action of $\mathfrak{G}(\Phi)$ on $\mathfrak{X}$ is locally quasi-analytic.

Section 6 recalls the notion of a continuous orbit equivalence (COE) between Cantor actions, then discusses three notions of rigidity for Cantor actions. The work of $\mathrm{Li}$ in 38 gives cohomological criteria for when two COE Cantor actions, both of which are topologically free actions, are necessarily $\theta$-conjugate, as defined in Definition 6.3. The work of Cortez and Medynets in 13 shows that two COE Cantor actions, both of which are free, are structurally conjugate (or virtually rigid as defined in Definition 6.7). The notion of return equivalence for Cantor actions was introduced in [10, and the authors showed in [36] that two COE Cantor actions, both of which are stable, are necessarily return equivalent as in Definition 6.8.

It is thus natural to ask how the invariants $\Upsilon_{c}(\Phi)$ and $\Upsilon_{s}(\Phi)$ behave under orbit equivalence. Our first result, and technically most involved, shows the following:

THEOREM 1.4 (Theorem 6.10). Let $(\mathfrak{X}, G, \Phi)$ and $\left(\mathfrak{X}^{\prime}, G^{\prime}, \Psi\right)$ be continuously orbit equivalent Cantor actions. If $G$ is finitely generated, and $\left(\mathfrak{X}^{\prime}, G^{\prime}, \Psi\right)$ is stable, then $(\mathfrak{X}, G, \Phi)$ is stable.

We deduce from this a strong form of invariance for the wild property.

COROLLARY 1.5. The wild property is an invariant of continuous orbit equivalence for the class of Cantor actions by finitely generated groups.

Combining Corollary 1.5 with the results in [36] yields:

COROLLARY 1.6 (Theorem6.18). Let $G$ and $G^{\prime}$ be a finitely generated groups, and suppose that the Cantor action $\left(\mathfrak{X}^{\prime}, G^{\prime}, \Psi\right)$ is stable. Let $(\mathfrak{X}, G, \Phi)$ be a general Cantor action which is continuously orbit equivalent to $\left(\mathfrak{X}^{\prime}, G^{\prime}, \Psi\right)$, then the actions are return equivalent.

The above results give effective approaches to the classification of stable Cantor actions, up to conjugacy or continuous orbit equivalence. On the other hand, for wild Cantor actions, much less is known. The remainder of this work then investigates the properties of wild Cantor actions.

Section 7 introduces the notion of a non-Hausdorff element in $\mathfrak{G}(\Phi)$ for a Cantor action $(\mathfrak{X}, G, \Phi)$. It was remarked by Renault in [49 that if the germinal groupoid associated to a group action is non-Hausdorff, then the action cannot be topologically free. In the authors' work [36] this result was extended to the observation that if $\mathfrak{G}(\Phi)$ contains a non-Hausdorff element, then the action is wild. In this work, we give a more precise consequence. The stabilizer limit group $\Upsilon_{s}(\Phi)$ is said to be of finite type if each group in a representative group chain is a finite group. Then we have the following result, which relates the direct limit invariants and other ideas introduced in this paper with the dynamics of a Cantor action: 
THEOREM 1.7. Let $(\mathfrak{X}, G, \Phi)$ be a Cantor action. Suppose that $\mathfrak{G}(\Phi)$ contains a non-Hausdorff element, then the action is dynamically wild and not of finite type. That is, there is a proper inclusion of direct limit groups $\Upsilon_{c}(\Phi) \subset \Upsilon_{s}(\Phi)$, and $\Upsilon_{s}(\Phi)$ is represented by an increasing chain of Cantor groups, which in particular are uncountable.

The claims of Theorem 1.7 follow from Corollary 7.4 and Theorem 7.5 ,

COROLLARY 1.8. Let $(\mathfrak{X}, G, \Phi)$ be a Cantor action for which the stabilizer direct limit group $\Upsilon_{s}(\Phi)$ has finite type, then the germinal groupoid $\mathcal{G}(\mathfrak{X}, G, \Phi)$ associated to the action is Hausdorff.

The examples of wild Cantor actions given in [35, Section 8] have finite type, so Corollary 1.8 implies there exists wild Cantor actions with Hausdorff germinal groupoids.

It seems to be a difficult problem to give criteria for a dynamically wild Cantor action which suffice to imply the existence of a non-Hausdorff element. Section 8 constructs examples of wild Cantor actions using the "automata" approach, which is a well-known method in Geometric Group Theory. This method defines a homeomorphism of the boundary of a tree, using a recursive definition along the branches of the tree. The examples constructed are inspired by the work of Nekrashevych in [44, 45, and Pink in [46. It would be very interesting to know if other methods of construction are possible, and perhaps that the presence of a non-Hausdorff element for a Cantor action implies some form of underlying recursiveness for the action of its generators.

We conclude with the two general problems most relevant to the results of this work.

PROBLEM 1.9. Give sufficient conditions for a pair $(\mathfrak{G}, \mathcal{D})$ to admit a non-Hausdorff element for the action of $\mathfrak{G}$ on $\mathfrak{X} \equiv \mathfrak{G} / \mathcal{D}$, where $\mathfrak{G}$ is a profinite group which is finitely generated in the topological sense, and $\mathcal{D} \subset \mathfrak{G}$ is a "totally not normal" closed subgroup.

The notion of a totally not normal subgroup is given in Definition 2.2 .

Note that this problem is not just about the algebraic properties of the groups, as the wild property only emerges when considering the transitive action of $\mathfrak{G}$ on the quotient space $\mathfrak{X}$.

For the examples of wild Cantor actions of flat type given in [35, Section 8], the discriminant groups of these actions are an infinite product of finite groups. The second question asks whether there is a general result, that the discriminant groups of flat actions have a restricted algebraic structure.

PROBLEM 1.10. Let $(\mathfrak{X}, G, \Phi)$ be a wild Cantor action of flat type, so the inclusion of direct limit groups $\Upsilon_{c}(\Phi) \subset \Upsilon_{s}(\Phi)$ is an equality. What restrictions are imposed on its discriminant group?

\section{THE PROFINITE MODEL}

Given a Cantor action $(\mathfrak{X}, G, \Phi)$, let $\Phi(G) \subset$ Homeo $(X)$ denote the image subgroup. Introduce the closure $\mathfrak{G}(\Phi) \equiv \overline{\Phi(G)} \subset$ Homeo $(X)$ for the uniform topology of maps. (This corresponds to the Ellis group for the action, as defined in [3, 21, 22, see also [17, Section 2].) That is, each element $\widehat{g} \in \mathfrak{G}(\Phi)$ is the uniform limit of a sequence of maps $\left\{\Phi\left(g_{i}\right) \mid i \geq 1\right\} \subset \Phi(G)$. By abuse of notation, we sometimes also denote the limiting element $\widehat{g}$ by $\left(g_{i}\right)$. It was observed by Ellis in [21, by Auslander in [3, Chapter 3] and again by Glasner in [32, Proposition 2.5], that the assumption the action is equicontinuous implies $\mathfrak{G}(\Phi)$ is a separable profinite group.

For example, if $G$ is an abelian group, then $\mathfrak{G}(\Phi)$ is a compact totally disconnected abelian group, which can be thought of as the group of asymptotic motions of the system. When $G$ is non-abelian, the action closure $\mathfrak{G}(\Phi)$ can have more subtle algebraic properties. A main theme of this work is to examine the interplay between the algebraic structure of $\mathfrak{G}(\Phi)$ and the dynamics of the action $\Phi$.

The philosophy behind our study of Cantor actions, is to consider $\mathfrak{X}$ as a homogeneous space for $\mathfrak{G}(\Phi)$, in analogy to the case of homogeneous spaces of Lie type. Recall that for $G$ a connected Lie group and $H \subset G$ a closed subgroup, the quotient space $X=G / H$ is a homogeneous $G$-space, and one studies the geometry of $X$ using the structure of the Lie algebra $\mathfrak{g}$ of $G$, and the adjoint action 
of $H$ on $\mathfrak{g}$. See for example Chapters $\mathrm{X}$ and XI of 37 . For a Cantor action $(\mathfrak{X}, G, \Phi)$, there is no obvious analog of a Lie algebra associated to $\mathfrak{G}(\Phi)$. None-the-less, one can investigate the properties of the adjoint action of the isotropy group of a point, localized to neighborhoods of $x$.

Let $\widehat{\Phi}: \mathfrak{G}(\Phi) \times \mathfrak{X} \rightarrow \mathfrak{X}$ denote the induced action of $\mathfrak{G}(\Phi)$ on $\mathfrak{X}$. For $\widehat{g} \in \mathfrak{G}(\Phi)$ we write its action on $\mathfrak{X}$ by $\widehat{g} \cdot x=\widehat{\Phi}(\widehat{g})(x)$. If the action $\Phi: G \times \mathfrak{X} \rightarrow \mathfrak{X}$ is minimal, then the group $\mathfrak{G}(\Phi)$ acts transitively on $\mathfrak{X}$. The action $(\mathfrak{X}, G, \Phi)$ is said to be faithful if $\Phi(g) \cdot x=x$ for all $x \in \mathfrak{X}$ implies that $g$ is the identity element. Equivalently, the action is faithful if the action map $\Phi: G \rightarrow \operatorname{Homeo}(\mathfrak{X})$ is injective. The action is free if for any $x \in \mathfrak{X}, \Phi(g) \cdot x=x$ implies that $g$ is the identity element of $G$.

2.1. The adjoint action. Given $x \in \mathfrak{X}$, introduce the isotropy group at $x$,

$$
\mathfrak{G}(\Phi)_{x}=\{\widehat{g} \in \overline{\Phi(G)} \mid \widehat{g} \cdot x=x\} \subset \operatorname{Homeo}(\mathfrak{X}),
$$

which is a closed subgroup of $\mathfrak{G}(\Phi)$, and is thus either finite, or is a Cantor group.

There is a natural identification $\mathfrak{X} \cong \mathfrak{G}(\Phi) / \mathfrak{G}(\Phi)_{x}$ of left $G$-spaces, and thus the conjugacy class of $\mathfrak{G}(\Phi)_{x}$ in $\mathfrak{G}(\Phi)$ is independent of the choice of $x$. Moreover, if $\mathfrak{G}(\Phi)_{x}$ is the trivial group, then $\mathfrak{X}$ is identified with a profinite group, and the action is free. However, there exists examples of free Cantor actions for which the group $\mathfrak{G}(\Phi)_{x}$ is non-trivial; the first such examples were constructed by Fokkink and Oversteegen in [25, Section 8], and further examples were constructed in [19, Section 10].

The action of $\mathfrak{G}(\Phi)_{x}$ on $\mathfrak{X}$ can be considered as induced by the adjoint action of $\mathfrak{G}(\Phi)_{x}$ on $\mathfrak{G}(\Phi)$. For $\widehat{h} \in \mathfrak{G}(\Phi)_{x}$ and $\widehat{g} \in \mathfrak{G}(\Phi)$, let $\operatorname{Ad}(\widehat{h})(\widehat{g})=\widehat{h} \widehat{g} \widehat{h}^{-1}$. For $y \in \mathfrak{X}$ choose $\widehat{g} \in \mathfrak{G}(\Phi)$ such that $y=\widehat{g} \cdot \mathfrak{G}(\Phi)_{x}$. Then for $\widehat{h} \in \mathfrak{G}(\Phi)_{x}$ we have

$$
\widehat{h} \cdot y=\widehat{h} \widehat{g} \cdot \mathfrak{G}(\Phi)_{x}=\widehat{h} \widehat{g} \widehat{h}^{-1} \cdot \mathfrak{G}(\Phi)_{x}=\mathbf{A d}(\widehat{h})(\widehat{g}) \cdot \mathfrak{G}(\Phi)_{x} .
$$

That is, the action of $\mathfrak{G}(\Phi)_{x}$ on $\mathfrak{X}$ can be considered as the factor of the adjoint action which is "normal" to $\mathfrak{G}(\Phi)_{x}$. In the case of a homogeneous space of Lie type, this normal action is induced by the adjoint action on the Lie algebra of $\mathfrak{G}(\Phi)$, and is studied in terms of representation theory of the compact group $\mathfrak{G}(\Phi)_{x}$. For a Cantor action, we instead consider the restriction of the adjoint action to arbitrarily small neighborhoods of $x \in \mathfrak{X}$. If this localized action of $\mathfrak{G}(\Phi)_{x}$ stabilizes for arbitrarily small neighborhoods of $x$, then the action is said to be stable, and otherwise it is wild. That is, for a stable Cantor action, there is a well-defined local geometric model for $\mathfrak{X}$ near $x$, while for a wild action there is no stable local model. We next make these statements precise.

2.2. The finite model. A profinite group, by definition, is the inverse limit of finite quotient groups. For an equicontinuous action $(\mathfrak{X}, G, \Phi)$, an analogous statement holds, that the action $\Phi$ is defined by an inverse limit of finite actions. The key concept to show this is that of clopen subsets of $\mathfrak{X}$ which are adapted to the action. We briefly recall some basic concepts.

Let $\mathrm{CO}(\mathfrak{X})$ denote the collection of all clopen (closed and open) subsets of the Cantor space $\mathfrak{X}$, which forms a basis for the topology of $\mathfrak{X}$. For $\phi \in \operatorname{Homeo}(\mathfrak{X})$ and $U \in \mathrm{CO}(\mathfrak{X})$, the image $\phi(U) \in \mathrm{CO}(\mathfrak{X})$. The following result is folklore, and a proof is given in [36, Proposition 3.1].

PROPOSITION 2.1. A minimal Cantor action $(\mathfrak{X}, G, \Phi)$ is equicontinuous if and only if, for the induced action $\Phi_{*}: G \times \mathrm{CO}(\mathfrak{X}) \rightarrow \mathrm{CO}(\mathfrak{X})$, the $G$-orbit of every $U \in \mathrm{CO}(\mathfrak{X})$ is finite.

The proof that each $U \in \mathrm{CO}(\mathfrak{X})$ has finite orbit is essentially the same as what was called the "coding method" for equicontinuous pseudogroup actions on Cantor transversals in [9, and discussed for group actions in [17, Appendix A], and for free actions in [13, Section 2].

We say that $U \subset \mathfrak{X}$ is adapted to the action $(\mathfrak{X}, G, \Phi)$ if $U$ is a non-empty clopen subset, and for any $g \in G$, if $\Phi(g)(U) \cap U \neq \emptyset$ implies that $\Phi(g)(U)=U$. The proof of Proposition 3.1 in 36 shows that given $x \in \mathfrak{X}$ and clopen set $x \in W$, there is an adapted clopen set $U$ with $x \in U \subset W$.

The key property of adapted sets, is that for $U$ adapted, the set of "return times" to $U$,

$$
G_{U}=\{g \in G \mid \varphi(g)(U) \cap U \neq \emptyset\}
$$


is a subgroup of $G$, called the stabilizer of $U$. Then for $g, g^{\prime} \in G$ with $g \cdot U \cap g^{\prime} \cdot U \neq \emptyset$ we have $g^{-1} g^{\prime} \cdot U=U$, hence $g^{-1} g^{\prime} \in G_{U}$. Thus, the translates $\{g \cdot U \mid g \in G\}$ form a finite clopen partition of $\mathfrak{X}$, and are in 1-1 correspondence with the quotient space $X_{U}=G / G_{U}$. Then $G$ acts by permutations of the finite set $X_{U}$ and so the stabilizer group $G_{U} \subset G$ has finite index. The action of $g \in G$ on $X_{U}$ is trivial precisely when $g$ is a stabilizer of each coset $h \cdot G_{U}$, so $g \in C_{U}$ where

$$
C_{U}=\bigcap_{h \in G} h G_{U} h^{-1} \subset G_{U}
$$

is the largest normal subgroup of $G$ contained in $G_{U}$. The action of the finite group $Q_{U} \equiv G / C_{U}$ on $X_{U}$ by permutations is a finite approximation of the action of $G$ on $\mathfrak{X}$, and the isotropy group of the identity coset $e \cdot G_{U}$ is $D_{U} \equiv G_{U} / C_{U} \subset Q_{U}$.

Thus, the finite model of a Cantor action $(\mathfrak{X}, G, \Phi)$ obtained from $U \in \operatorname{CO}(\mathfrak{X})$ yields the following data: The group $Q_{U}$ which acts on $X_{U}$, and the isotropy group $D_{U} \subset Q_{U}$ for the basepoint defined by the identity coset $x_{U}=e \cdot G_{U} \in X_{U}$. In addition to the group structures of $Q_{U}$ and $D_{U}$, one can study how the group $D_{U}$ is "algebraically embedded" in $Q_{U}$.

Consider the adjoint action of $D_{U}$ on $Q_{U}$, which for $h \in D_{U}$ is given by

$$
\operatorname{Ad}_{Q_{U}}(h): Q_{U} \rightarrow Q_{U} \quad, \quad \operatorname{Ad}_{Q_{U}}(h)(k)=h k h^{-1}, k \in Q_{U} .
$$

The subgroup $D_{U}$ is not a normal subgroup in $Q_{U}$, and in fact a stronger condition holds. Let $h \in D_{U}$ and suppose that $k h k^{-1} \in D_{U}$ for all $k \in Q_{U}$. Then $k h k^{-1}=h^{\prime}$ for some $h^{\prime} \in D_{U}$, so $h k^{-1}=k^{-1} h^{\prime}=k^{-1} h^{\prime \prime} h$ for $h^{\prime \prime}=h^{\prime} h^{-1}$. Hence $h k^{-1} h^{-1}=k^{-1} h^{\prime \prime}$. As this holds for all $k \in Q_{U}$ the adjoint $\operatorname{Ad}_{Q_{U}}(h)$ acts trivially on $X_{U}$ and hence $h$ must be the identity in $Q_{U}$. In other words, for every non-trivial $h \in D_{U}$, there is a $k \in Q_{U}$ such that $k h k^{-1} \notin D_{U}$. This shows that the subgroup $D_{U}$ satisfies the following condition, which was introduced in the works [17, 35].

DEFINITION 2.2. A subgroup $H \subset G$ is said to be totally not normal if for each non-trivial $h \in H$, there exists $g \in G$ such that $g h g^{-1} \notin H$.

2.3. Induced actions. We next consider the relations between the finite model constructed in Section 2.2 and the actions of $G$ and $\mathfrak{G}(\Phi)$ on $\mathfrak{X}$. We first establish some technical results about restriction maps for adapted sets, then consider the behavior of the adjoint map for $\widehat{h} \in \mathfrak{G}(\Phi)_{x}$ under restriction to adapted sets for the action. Let $U$ be adapted to the Cantor action $(\mathfrak{X}, G, \Phi)$.

The first observation is that each $\widehat{g} \in \mathfrak{G}(\Phi)$ is the uniform limit of a sequence of maps, $\widehat{g}=\left(g_{i}\right)$, and the translates of $U$ form a finite partition of $\mathfrak{X}$ by closed sets, hence the action of $\widehat{g}$ on the partition $X_{U}$ equals the action by $g_{i}$ for $i$ sufficiently large. Thus, there is a well-defined epimorphism $\widehat{q}_{U}: \mathfrak{G}(\Phi) \rightarrow Q_{U}$, which maps $\mathfrak{G}(\Phi)_{x}$ onto $D_{U}$ for $x \in U$.

Let $\left(U, G_{U}, \Phi_{U}\right)$ denote the action of $G_{U}$ restricted to $U$, which is again a Cantor action. Define

$$
H_{U} \equiv \Phi_{U}\left(G_{U}\right) \subset \operatorname{Homeo}(U) \quad, \quad K_{U}=\operatorname{ker}\left\{\Phi_{U}: G_{U} \rightarrow H_{U}\right\} \subset G_{U} \subset G .
$$

LEMMA 2.3. The action of $H_{U}$ on $U$ is minimal.

Proof. As $U$ is adapted, for each $x \in U$, the translates $H_{U} \cdot x=\left\{g \cdot x \in U \mid g \in G_{U}\right\}$ are dense.

The group $H_{U}$ is called the holonomy of the action $\Phi$ restricted to $U$, in analogy with the case of actions which arise from the holonomy of weak solenoids [18, 19. For each $g \in K_{U}$ the action $\Phi(g)$ fixes every point in $U$. Note that $K_{\mathfrak{X}}$ is the trivial group exactly when $\Phi$ is a faithful action, and $K_{U}$ can be non-trivial even when the action $\Phi$ on $\mathfrak{X}$ is faithful.

Next, consider the closures of the restricted groups defined in (6). There is a subtle point to consider, that the closure can either be taken in the uniform topology on $\operatorname{Homeo}(\mathfrak{X})$, or after restriction, in the uniform topology on $\operatorname{Homeo}(U)$. Let $\widehat{H}_{U}=\overline{\Phi_{U}\left(G_{U}\right)} \subset \operatorname{Homeo}(U)$ denote the closure of $H_{U}$ in the uniform topology on $\operatorname{Homeo}(U)$. Then Lemma 2.3 implies:

COROLLARY 2.4. Let $U \subset \mathfrak{X}$ be adapted, then $\widehat{H}_{U}$ acts transitively on $U$. 
The following is a key technical observation, and is often used implicitly in the following.

LEMMA 2.5. Let $U \subset \mathfrak{X}$ be adapted for the Cantor action $(\mathfrak{X}, G, \Phi)$. Then

$$
\widehat{H}_{U}=\{\widehat{g}|U| \widehat{g} \in \mathfrak{G}(\Phi), \widehat{g} \cdot U=U\} .
$$

Proof. Let $\widehat{h}=\left(g_{i}\right) \in \widehat{H}_{U}$ for $g_{i} \in G_{U}$, where the sequence $\left\{\Phi_{U}\left(g_{i}\right)\right\}$ converges in the uniform topology in Homeo $(U)$. Since $\mathfrak{G}(\Phi)$ is compact, there exists a subsequence $\left\{g_{i_{\ell}} \mid \ell \geq 0\right\}$ such that $\left\{\Phi\left(g_{i_{\ell}}\right)\right\}$ converges uniformly in Homeo $(\mathfrak{X})$, hence yields $\widehat{g}=\left(g_{i_{\ell}}\right) \in \mathfrak{G}(\Phi)$. Moreover, as $U$ is adapted, we can assume that each $\Phi\left(g_{i_{\ell}}\right)(U)=U$, so $\widehat{g} \cdot U=U$. Then $\widehat{g} \mid U=\widehat{h}$, as uniform convergence on $\mathfrak{X}$ implies uniform convergence on $U$, hence the actions agree on $U$. This shows that $\widehat{H}_{U} \subset\{\widehat{g}|U| \widehat{g} \cdot U=U\}$. The converse is immediate, since $\widehat{g} \in \mathfrak{G}(\Phi)$ with $\widehat{g} \cdot U=U$ implies, as noted above, that we can assume $\widehat{g}=\left(g_{i}\right)$ where each $\Phi\left(g_{i}\right)(U)=U$, hence $\widehat{g} \mid U \in \widehat{H}_{U}$.

For adapted sets $V \subset U$, introduce the subgroups

(8) $\widehat{H}_{U, V} \equiv\left\{\widehat{h} \in \widehat{H}_{U} \mid \widehat{h} \cdot V=V\right\} \subset \operatorname{Homeo}(U) \quad, \quad \widehat{K}_{U, V} \equiv\left\{\widehat{h} \in \widehat{H}_{U, V}|\widehat{h}| V=i d\right\} \subset \operatorname{Homeo}(U)$.

Note that $\widehat{H}_{\mathfrak{X}, U} \subset \operatorname{Homeo}(\mathfrak{X})$ while $\widehat{H}_{U} \subset \operatorname{Homeo}(U)$, and Lemma 2.5] implies there is a surjective restriction map $\rho_{U}: \widehat{H}_{\mathfrak{X}, U} \rightarrow \widehat{H}_{U}$.

LEMMA 2.6. Let $U \subset \mathfrak{X}$ be adapted for the Cantor action $(\mathfrak{X}, G, \Phi)$ with $x \in U$. Then

$$
\widehat{K}_{\mathfrak{X}, U} \subset \mathfrak{G}(\Phi)_{x} \subset \widehat{H}_{\mathfrak{X}, U} \subset \mathfrak{G}(\Phi) \text {. }
$$

Proof. For $\widehat{h} \in \widehat{K}_{\mathfrak{X}, U}$ then $\widehat{h} \cdot x=x$, hence $\widehat{h} \in \mathfrak{G}(\Phi)_{x}$. For $\widehat{h} \in \mathfrak{G}(\Phi)_{x}$ we have $\widehat{h} \cdot U \cap U \neq \emptyset$, hence $\widehat{h} \cdot U=U$ and so $\widehat{h} \in \widehat{H}_{\mathfrak{X}, U}$.

2.4. Induced adjoint actions. For $U \subset \mathfrak{X}$ adapted with $x \in U$, we consider the restriction of the adjoint action of $\mathfrak{G}(\Phi)_{x}$ to the subgroup $\widehat{H}_{\mathfrak{X}, U} \subset \mathfrak{G}(\Phi)$. Let $\widehat{h} \in \mathfrak{G}(\Phi)_{x}$ then $\widehat{h}_{\boldsymbol{h}} \widehat{H}_{\mathfrak{X}, U}$ by Lemma 2.6. so the adjoint action of $\widehat{h}$ restricts to an action $\operatorname{Ad}_{U}(\widehat{h}): \widehat{H}_{\mathfrak{X}, U} \rightarrow \widehat{H}_{\mathfrak{X}, U}$. Consider the induced action on the quotient space, $\operatorname{Ad}_{U}(\widehat{h}): U \rightarrow U$.

LEMMA 2.7. Let $U \in \mathrm{CO}(\mathfrak{X}), x \in U$. Then $\widehat{h} \in \mathfrak{G}(\Phi)_{x}$ acts as the identity on $U$ if and only if

$$
\operatorname{Ad}_{U}(\widehat{h})(\widehat{g}) \cdot \mathfrak{G}(\Phi)_{x}=\widehat{g} \cdot \mathfrak{G}(\Phi)_{x}, \text { for all } \widehat{g} \in \widehat{H}_{\mathfrak{X}, U}
$$

Proof. Suppose that $\widehat{h}$ acts as the identity on $U$, so $\widehat{h} \in \mathfrak{G}(\Phi)_{x} \cap \widehat{K}_{\mathfrak{X}, U}$. For $y \in U$, by Corollary 2.4 we can choose $\widehat{g} \in \mathfrak{G}(\Phi)$ such that $y=\widehat{g} \cdot \mathfrak{G}(\Phi)_{x}$. Then $\widehat{g} \cdot U \cap U \neq \emptyset$, hence $\widehat{g} \in \widehat{H}_{\mathfrak{X}, U}$ and since $\widehat{h} \cdot y=y$, we have

$$
\operatorname{Ad}_{U}(\widehat{h})(\widehat{g}) \cdot \mathfrak{G}(\Phi)_{x}=\widehat{h} \widehat{g} \widehat{h}^{-1} \cdot \mathfrak{G}(\Phi)_{x}=\widehat{h} \widehat{g} \cdot \mathfrak{G}(\Phi)_{x}=\widehat{h} \cdot y=y=\widehat{g} \cdot \mathfrak{G}(\Phi)_{x},
$$

which yields the identity (10). Conversely, if $\widehat{h} \in \mathfrak{G}(\Phi)_{x}$ satisfies (10), then (11) implies that $\widehat{h} \cdot y=y$ and that $\widehat{h} \in \mathfrak{G}(\Phi)_{x} \cap \widehat{K}_{\mathfrak{X}, U}$.

Let us also introduce the adjoint automorphisms on the isotropy subgroup $\mathfrak{G}(\Phi)_{x}$. For $\widehat{h} \in \mathfrak{G}(\Phi)_{x}$ let $\operatorname{Ad}_{x}(\widehat{h}): \mathfrak{G}(\Phi)_{x} \rightarrow \mathfrak{G}(\Phi)_{x}$ denote the adjoint action, given by $\mathbf{A d}_{x}(\widehat{h})(\widehat{k})=\widehat{h} \widehat{k} \widehat{h}^{-1}$ for $\widehat{k} \in \mathfrak{G}(\Phi)_{x}$.

REMARK 2.8. Note that the condition (10) does not imply that $\mathbf{A d}_{x}(\widehat{h})$ acts as the identity on the isotropy subgroup $\mathfrak{G}(\Phi)_{x}$ as the action of $\mathbf{A d}_{x}(\widehat{h})$ is absorbed when passing to cosets of $\mathfrak{G}(\Phi)_{x}$. Examples show that the action of $\mathbf{A d}_{x}(\widehat{h})$ can be non-trivial, while the induced quotient action on $U$ is trivial. This observation lies behind the distinction between the definitions of the stabilizer and centralizer limit groups in Section 4.2, and the notions "wild of flat type" and "dynamically wild" actions in Definition 4.18. 


\section{Odometer Models}

The odometer model for a Cantor action $(\mathfrak{X}, G, \Phi)$ is obtained from a sequence of finite approximations of the action, as in Section 2. For example, a Vietoris solenoid is defined as the inverse limit of a sequence of finite coverings of $\mathbb{S}^{1}$, which is equivalent to considering a descending chain of finite index subgroups of the fundamental group $\mathbb{Z}$ of $\mathbb{S}^{1}$. For a Cantor action, one considers a descending chain of finite index subgroups of a fixed group $G$, and then forms the inverse limit action associated to this chain. The odometer model for an action can be thought of as an "algebraic model" for the action, which enables relating the algebraic properties of $G$ with the dynamics of its action.

Downarowicz gives a survey of classical odometers in [16]. Some properties of odometer models for actions of non-abelian groups $G$ are discussed by Cortez and Petite in the works [12, 14, and by the authors in [17, 19, 35. Section 2 of [13] discusses the literature for non-abelian odometers.

We first describe the construction of the odometer model as the inverse limit action obtained from finite approximations associated to adapted subsets of $\mathfrak{X}$. We then consider the equivalence of these odometer models, and finally consider the adjoint action for these inverse limit actions.

3.1. Group chains. For a choice of basepoint $x \in \mathfrak{X}$ and $\varepsilon>0$, Proposition 2.1 implies there exists an adapted clopen set $U \in \mathrm{CO}(\mathfrak{X})$ with $x \in U$ and $\operatorname{diam}(U)<\varepsilon$. Thus, given a basepoint $x$, by iterating this process one can always construct the following:

DEFINITION 3.1. Let $(\mathfrak{X}, G, \Phi)$ be a Cantor action. A properly descending chain of clopen sets $\mathcal{U}=\left\{U_{\ell} \subset \mathfrak{X} \mid \ell \geq 0\right\}$ is said to be an adapted neighborhood basis at $x \in \mathfrak{X}$ for the action $\Phi$ if $x \in U_{\ell+1} \subset U_{\ell}$ for all $\ell \geq 1$ with $\bigcap_{\ell \geq 1} U_{\ell}=\{x\}$, and each $U_{\ell}$ is adapted to the action $\Phi$.

Given an adapted neighborhood basis $\mathcal{U}$ at $x$, for each $\ell \geq 0$ we can repeat the constructions of Section 2 for the adapted set $U_{\ell}$. As a matter of notation, after fixing the collection $\mathcal{U}$, we use the subscript $\ell$ in place of the subscript $U_{\ell}$ when convenient. For example, $G_{\ell} \equiv G_{U_{\ell}}$ denotes the stabilizer group of $U_{\ell}$. Then we obtain a descending chain of finite index subgroups

$$
\mathcal{G}_{\mathcal{U}}^{x}=\left\{G=G_{0} \supset G_{1} \supset G_{2} \supset \cdots\right\}
$$

Note that each $G_{\ell}$ has finite index in $G$, and is not assumed to be a normal subgroup. Also note that while the intersection of the chain $\mathcal{U}$ is a single point $\{x\}$, the intersection of the stabilizer groups in $\mathcal{G}_{\mathcal{U}}^{x}$ need not be the trivial group.

Next, set $X_{\ell}=G / G_{\ell}$ with basepoint $x_{\ell}=e G_{\ell} \in X_{\ell}$, where $e \in G$ is the identity element. Note that $G$ acts transitively on the left on $X_{\ell}$. The inclusion $G_{\ell+1} \subset G_{\ell}$ induces a natural $G$-invariant quotient map $p_{\ell+1}: X_{\ell+1} \rightarrow X_{\ell}$. Introduce the inverse limit

$$
X_{\infty} \equiv \lim _{\longleftarrow}\left\{p_{\ell+1}: X_{\ell+1} \rightarrow X_{\ell} \mid \ell>0\right\}
$$

which is a Cantor space with the Tychonoff topology, and basepoint $x_{\infty}=\left(x_{\ell}\right)$. The actions of $G$ on the factors $X_{\ell}$ induce a minimal equicontinuous action on $X_{\infty}$, denoted by $\Phi_{x}: G \times X_{\infty} \rightarrow X_{\infty}$.

For each $\ell \geq 0$, we have the "partition coding map" $\Theta_{\ell}: \mathfrak{X} \rightarrow X_{\ell}$ which is $G$-equivariant. The maps $\left\{\Theta_{\ell}\right\}$ are compatible with the quotient maps in (13), and so induce a limit map $\Theta_{x}: \mathfrak{X} \rightarrow X_{\infty}$. The fact that the diameters of the clopen sets $\left\{U_{\ell}\right\}$ tend to zero, implies that $\Theta_{x}$ is a homeomorphism. This is proved in detail in [17. Appendix A]. Moreover, $\Theta_{x}(x)=x_{\infty} \in X_{\infty}$, the basepoint of the inverse limit (13). Let $X_{\infty}$ have a metric such that $G$ acts on $X_{\infty}$ by isometries, then let $d_{\mathfrak{X}}$ be the metric on $\mathfrak{X}$ induced by the homeomorphism $\widehat{\Theta}_{x}$. The minimal equicontinuous action $\left(X_{\infty}, G, \Phi_{x}\right)$ is called the odometer model centered at $x$ for the action $(\mathfrak{X}, G, \Phi)$.

3.2. The Ellis group. We next introduce the group chain model for the closure group $\mathfrak{G}(\Phi)$, as the inverse limit of the finite models for the action $\Phi$ introduced in Section 2 . For each $\ell \geq 1$, let $C_{\ell}$ 
denote the largest normal subgroup (the core) of the stabilizer group $G_{\ell}$, so

$$
C_{\ell}=\bigcap_{g \in G} g G_{\ell} g^{-1} \subset G_{\ell} .
$$

As $G_{\ell}$ has finite index in $G$, the same holds for $C_{\ell}$. Observe that for all $\ell \geq 0$, we have $C_{\ell+1} \subset C_{\ell}$. Introduce the quotient group $Q_{\ell}=G / C_{\ell}$ with identity element $e_{\ell}=e C_{\ell} \in Q_{\ell}$. Then $Q_{\ell}$ acts transitively on the quotient set $X_{\ell}=G / G_{\ell}$. Let $\pi_{\ell+1}: Q_{\ell+1} \rightarrow Q_{\ell}$ be the quotient map induced by the inclusion $C_{\ell+1} \subset C_{\ell}$, which is equivariant for the left $G$-actions. Form the inverse limit group,

$$
\widehat{G}_{\infty} \equiv \lim _{\longleftarrow}\left\{\pi_{\ell+1}: Q_{\ell+1} \rightarrow Q_{\ell} \mid \ell \geq 0\right\} \subset \prod_{\ell \geq 0} Q_{\ell} .
$$

For $\ell \geq 0$, let $\widehat{q}_{\ell}: \widehat{G}_{\infty} \rightarrow Q_{\ell}$ be the projection map onto the $\ell$-th factor. Let $\widehat{C}_{\ell}$ denote its kernel.

Give $\widehat{G}_{\infty}$ the relative topology induced by the product (Tychonoff) topology; that is, a basis for the topology of $\widehat{G}_{\infty}$ is given by the preimages of points for the maps $\widehat{q}_{\ell}$ for $\ell \geq 1$. For $\ell \geq 1$,

$$
\widehat{C}_{\ell}=\widehat{q}_{\ell}^{-1}\left(e_{\ell}\right)=\left\{\left(g_{i}^{\prime}\right) \in \widehat{G}_{\infty} \mid g_{i}^{\prime} C_{i}=e_{i} \text { for } 0 \leq i \leq \ell\right\}
$$

is a clopen neighborhood of $\widehat{e}=\left(e_{\ell}\right) \in \widehat{G}_{\infty}$. Then for any $\widehat{g} \in \widehat{G}_{\infty}$, the collection $\left\{\widehat{g} \widehat{C}_{\ell} \mid \ell \geq 0\right\}$ forms a neighborhood basis at $\widehat{g}$.

For each $\ell \geq 0$, let $\widehat{G}_{\ell} \subset \widehat{G}_{\infty}$ denote the clopen subgroup

$$
\widehat{G}_{\ell} \equiv \bigcup_{g \in G_{\ell} / C_{\ell}} g \widehat{C}_{\ell}=\bigcup_{g \in G_{\ell} / C_{\ell}} \widehat{C}_{\ell} g
$$

For $\ell \geq 0$, let $q_{\ell}: G \rightarrow Q_{\ell}$ be the quotient map. The sequence of maps $\left\{q_{\ell} \mid \ell \geq 0\right\}$ induces a homomorphism $q_{\infty}: G \rightarrow \widehat{G}_{\infty}$ for which $q_{\infty}(e)=\widehat{e}$. Let $\widehat{\Theta}: \mathfrak{G}(\Phi) \rightarrow \widehat{G}_{\infty}$ denote the closure of $q_{\infty}$ in the uniform topology on maps. We then have the folklore result (see [17, Theorem 4.4] for a proof):

THEOREM 3.2. Let $(\mathfrak{X}, G, \Phi)$ be a Cantor action, and suppose that $\left\{G_{\ell} \mid \ell \geq 0\right\}$ is the group chain associated to an adapted neighborhood basis $\mathcal{U}$ at $x \in \mathfrak{X}$. Then $\widehat{\Theta}: \mathfrak{G}(\Phi) \rightarrow \widehat{G}_{\infty}$ is an isomorphism of topological groups.

Recall that for $\ell \geq 0, D_{\ell} \equiv G_{\ell} / C_{\ell}$ is the isotropy group at the basepoint $x_{\ell}=e_{\ell} G_{\ell} \in X_{\ell}$ of the action of $Q_{\ell}$ on $X_{\ell}$. Moreover, by the definition of $\widehat{G}_{\infty}$, the quotient maps $\pi_{\ell+1}: Q_{\ell+1} \rightarrow Q_{\ell}$ in (15) are compatible with this identification.

LEMMA 3.3. The image $\widehat{\Theta}\left(\mathfrak{G}(\Phi)_{x}\right) \subset \widehat{G}_{\infty}$ is the subgroup defined by

$$
\mathcal{D}_{x} \equiv \lim _{\longleftarrow}\left\{\pi_{\ell+1}: G_{\ell+1} / C_{\ell+1} \rightarrow G_{\ell} / C_{\ell} \mid \ell \geq 0\right\} \subset \widehat{G}_{\infty} .
$$

Proof. For $h \in \mathfrak{G}(\Phi)_{x}$, let $h_{\ell}=\widehat{q}_{\ell}(\widehat{\Theta}(h)) \in Q_{\ell}$, then $\widehat{h}=\left(h_{\ell}\right) \in \widehat{G}_{\infty}$. The action of $h$ on $X_{\ell}$ fixes the coset $e_{\ell} G_{\ell}$, hence $h_{\ell} \in G_{\ell} / C_{\ell}$ for each $\ell \geq 1$. Moreover, $\pi_{\ell+1}\left(h_{\ell+1}\right)=h_{\ell}$ as the sequence $\left(h_{\ell}\right) \in \widehat{G}_{\infty}$. Thus, $\widehat{h} \in \mathcal{D}_{x}$ is well-defined in (18), and $\widehat{\Phi}_{x}(\widehat{h})\left(x_{\infty}\right)=x_{\infty}$. It follows that $\widehat{\Theta}\left(\mathfrak{G}(\Phi)_{x}\right) \subset \mathcal{D}_{x}$.

Conversely, given $\widehat{h}=\left(h_{\ell}\right) \in \mathcal{D}_{x}$, we have $\Phi\left(h_{\ell}\right) \in \operatorname{Homeo}(\mathfrak{X})$ for $\ell \geq 1$. Then by Theorem 3.2. the sequence $\left(\Phi\left(h_{\ell}\right)\right)$ of homeomorphisms of $\mathfrak{X}$ converges uniformly to a limit $\bar{h} \in \mathfrak{G}(\Phi)$. Note that multiplication by $\widehat{q}_{\ell}(\widehat{h}) \in Q_{\ell}$ fixes the coset $e G_{\ell}$, hence the action of $\bar{h}$ on $X_{\infty}$ leaves the clopen subset $U_{\ell} \subset X_{\infty}$ invariant for each $\ell \geq 0$. Hence, $\widehat{h} \cdot x=x$ so $\bar{h} \in \mathfrak{G}(\Phi)_{x}$.

Define $\widehat{\Theta}^{-1}: \mathcal{D}_{x} \rightarrow \mathfrak{G}(\Phi)_{x} \subset \operatorname{Homeo}(\mathfrak{X})$ by setting $\widehat{\Theta}^{-1}(\widehat{h})=\bar{h}$. By the construction, $\widehat{\Theta}$ and $\widehat{\Theta}^{-1}$ are inverse maps and are each continuous, thus $\widehat{\Theta}: \mathfrak{G}(\Phi)_{x} \rightarrow \mathcal{D}_{x}$ is a topological isomorphism.

The group $\mathcal{D}_{x}$ is called the discriminant of the action, in analogy with the interpretation of $\widehat{G}_{\infty}$ as the profinite Galois group associated to the sequence of irregular coverings $Q_{\ell} \rightarrow X_{\ell}$.

COROLLARY 3.4. The homeomorphism $\Theta_{x}: \mathfrak{X} \rightarrow X_{\infty}$ defined in Section 3.1 agrees with the induced map on quotients, $\widehat{\Theta}: \mathfrak{G}(\Phi) / \mathfrak{G}(\Phi)_{x} \rightarrow \widehat{G}_{\infty} / \mathcal{D}_{x}$. 
The abstract formula (18) is useful for computing the discriminant group, as in [17, Sections 6-8]. In particular, [17, Example 8.1] shows that the 3-dimensional Heisenberg group admits group chains for which $\mathcal{D}_{x}$ is a Cantor group.

The intersection $K\left(\mathcal{G}_{\mathcal{U}}^{x}\right)=\bigcap_{\ell \geq 0} G_{\ell}$ is called the kernel of $\mathcal{G}_{\mathcal{U}}^{x}$ and need not be the trivial group. Let $N K(\mathcal{G}) \subset K(\mathcal{G})$ denote the largest normal subgroup of $K(\mathcal{G})$, which is also characterized as the kernel of the homomorphism $q_{\infty}: G \rightarrow \widehat{G}_{\infty}$.

3.3. Equivalence of group chains. The construction of the odometer model for a Cantor action $(\mathfrak{X}, G, \Phi)$ depends upon the choice of a basepoint $x \in \mathfrak{X}$ and an adapted neighborhood basis $\mathcal{U}$ at $x$, which then yields the group chain $\mathcal{G}_{\mathcal{U}}^{x}$ used for the construction. It is thus important to understand how the group chain $\mathcal{G}_{\mathcal{U}}^{x}$ depends upon these choices. This leads to introducing notions of equivalence between group chains.

Let $G$ be a fixed group. A group chain in $G$ is a sequence of nested subgroups

$$
\mathcal{G}=\left\{G=G_{0} \supset G_{1} \supset G_{2} \supset \cdots\right\}
$$

where each $G_{\ell+1}$ has finite index in $G_{\ell}$ for $\ell \geq 0$. The first notion of equivalence between group chains was used by Rogers and Tollefson in their study of equivalence of weak solenoids in [50, and corresponds to the standard concept of chain equivalence in terms of interlacing of the chains.

DEFINITION 3.5. Two group chains $\left\{G_{\ell} \mid \ell \geq 0\right\}$ and $\left\{H_{\ell} \mid \ell \geq 0\right\}$ with $G_{0}=H_{0}=G$ are equivalent, if and only if, there is a group chain $\left\{K_{\ell} \mid \ell \geq 0\right\}$ in $G$, and infinite subsequences $\left\{G_{\ell_{k}}\right\}_{k \geq 0}$ and $\left\{H_{j_{k}}\right\}_{k \geq 0}$ such that $K_{2 k}=G_{\ell_{k}}$ and $K_{2 k+1}=H_{j_{k}}$ for $k \geq 0$.

For example, suppose that $\mathcal{U}$ and $\mathcal{U}^{\prime}$ are two choices of adapted neighborhood bases at $x$, then the corresponding group chains $\mathcal{G}_{\mathcal{U}}^{x}$ and $\mathcal{G}_{\mathcal{U}^{\prime}}^{x}$ are equivalent in this sense [25, 17.

The second notion of equivalence of group chains in $G$ is a generalization of the above notion, and was introduced by Fokkink and Oversteegen in [25], and further developed in the authors' work [17].

DEFINITION 3.6. 25] Two group chains $\left\{G_{\ell} \mid \ell \geq 0\right\}$ and $\left\{H_{\ell} \mid \ell \geq 0\right\}$ with $G_{0}=H_{0}=G$ are conjugate equivalent if and only if there exists a sequence $\left(g_{\ell}\right) \subset G_{0}$ for which the compatibility condition $g_{\ell} G_{\ell}=g_{\ell+1} G_{\ell}$ for all $\ell \geq 0$ is satisfied, and such that the group chains $\left\{g_{\ell} G_{\ell} g_{\ell}^{-1} \mid \ell \geq 0\right\}$ and $\left\{H_{\ell} \mid \ell \geq 0\right\}$ are equivalent.

The relation between the equivalences in Definitions 3.5 and 3.6 and their corresponding odometer models is given by the following theorem, which follows from results in [25]. The following result is stated and proved in [17].

THEOREM 3.7. Let $\left\{G_{\ell} \mid \ell \geq 0\right\}$ and $\left\{H_{\ell} \mid \ell \geq 0\right\}$ be group chains $G_{0}=H_{0}=G$, and let

$$
X_{\infty}=\lim _{\longleftarrow}\left\{G / G_{\ell+1} \rightarrow G / G_{\ell} \mid \ell \geq 0\right\} \quad, \quad Y_{\infty}=\lim _{\longleftarrow}\left\{G / H_{\ell+1} \rightarrow G / H_{\ell} \mid \ell \geq 0\right\} .
$$

Then the group chains $\left\{G_{\ell} \mid \ell \geq 0\right\}$ and $\left\{H_{\ell} \mid \ell \geq 0\right\}$ are equivalent if and only if there exists a homeomorphism $\tau: X_{\infty} \rightarrow Y_{\infty}$ equivariant with respect to the $G$-actions on $X_{\infty}$ and $Y_{\infty}$, and such that $\tau\left(e G_{\ell}\right)=\left(e H_{\ell}\right)$. That is, the conjugating map is basepoint-preserving.

The group chains $\left\{G_{\ell} \mid \ell \geq 0\right\}$ and $\left\{H_{\ell} \mid \ell \geq 0\right\}$ are conjugate equivalent if and only if there exists a homeomorphism $\tau: X_{\infty} \rightarrow Y_{\infty}$ equivariant with respect to the $G$-actions on $X_{\infty}$ and $Y_{\infty}$.

REMARK 3.8. Given a Cantor action $(\mathfrak{X}, G, \Phi)$, let $\mathcal{G}(\Phi)$ denote the group chains in $G$ which are conjugate equivalent to the group chain $\mathcal{G}_{\mathcal{U}}$ for some choice of basepoint $x \in \mathfrak{X}$ and adapted neighborhood basis $\mathcal{U}$ at $x$. Theorem 3.7 implies that an invariant defined for group chains in $G$, which is independent of the choice of a representative in $\mathcal{G}(\Phi)$, is an invariant for the conjugacy class of the action $(\mathfrak{X}, G, \Phi)$.

REMARK 3.9. Let $\mathcal{G}=\left\{G=G_{0} \supset G_{1} \supset G_{2} \supset \cdots\right\}$ be a group chain, with associated odometer $\left(X_{\infty}, G, \Phi_{x}\right)$. Let $N K(\mathcal{G}) \subset K(\mathcal{G})$ denote the largest normal subgroup of the kernel subgroup. It is immediate that $K(\mathcal{G})$ is an invariant of the equivalence class of $\mathcal{G}$, but examples show that it need not be invariant under conjugate equivalence. In contrast, it is immediate that $N K(\mathcal{G})$ is an 
invariant of conjugate equivalence, and is identified with the kernel of the action map $\Phi_{x}$ on $X_{\infty}$. Thus, $N K(\mathcal{G})$ is the trivial group whenever the action is faithful.

Applying these remarks to the group chains in $\mathcal{G}(\Phi)$ associated to a Cantor action $(\mathfrak{X}, G, \Phi)$, it follows that for a fixed basepoint $x \in \mathfrak{X}$, there is a well-defined kernel $\operatorname{subgroup} K\left(\mathcal{G}_{\mathcal{U}}^{x}\right) \subset G$. On the other hand, given another choice of basepoint $y \in \mathfrak{X}$, its kernel $K\left(\mathcal{G}_{\mathcal{U}}^{y}\right)$ need not equal $K\left(\mathcal{G}_{\mathcal{U}}^{x}\right)$. In fact, it is possible for kernel at $x$ to be the trivial group, and that at $y$ be a non-trivial subgroup. However, for free actions, they all agree and are trivial.

LEMMA 3.10. A Cantor action $(\mathfrak{X}, G, \Phi)$ is free if and only if, for all $x \in \mathfrak{X}$ and any adapted neighborhood basis $\mathcal{U}$ at $x$, the kernel $K\left(\mathcal{G}_{\mathcal{U}}^{x}\right)$ is the trivial group.

3.4. Adjoint group chains. We next consider the adjoint actions for the discriminant group of an odometer model for a Cantor action $(\mathfrak{X}, G, \Phi)$. Assume that we have fixed an adapted neighborhood basis $\mathcal{U}=\left\{U_{\ell} \subset \mathfrak{X} \mid \ell \geq 0\right\}$ at $x \in \mathfrak{X}$, and $\mathcal{G}_{\mathcal{U}}^{x}=\left\{G_{\ell} \mid \ell \geq 0\right\}$ is the associated group chain as in (12).

Recall that there are homeomorphisms $\mathfrak{X} \cong \mathfrak{G}(\Phi) / \mathfrak{G}(\Phi)_{x}$ and $X_{\infty} \cong \widehat{G}_{\infty} / \mathcal{D}_{x}$. By Theorem 3.2 there is an isomorphism $\widehat{\Theta}: \mathfrak{G}(\Phi) \rightarrow \widehat{G}_{\infty}$ of topological groups, which maps $\mathfrak{G}(\Phi)_{x}$ isomorphically to $\mathcal{D}_{x}$. For each $\ell \geq 0$, the clopen subgroup $\widehat{G}_{\ell} \subset \widehat{G}_{\infty}$ satisfies $\mathcal{D}_{x} \subset \widehat{G}_{\ell}$, and the quotient $\widehat{U}_{\ell} \equiv \widehat{G}_{\ell} / \mathcal{D}_{x} \subset \widehat{G}_{\infty} / \mathcal{D}_{x} \cong X_{\infty}$. Then Corollary 3.4 has a sharper statement, that for each $U_{\ell} \subset \mathfrak{X}$ in the adapted neighborhood basis $\mathcal{U}$ about $x$, we have $\Theta_{x}: U_{\ell} \rightarrow \widehat{U}_{\ell}$ is a homeomorphism.

We introduce the localizations of the adjoint actions of $\mathcal{D}_{x} \subset \widehat{G}_{\infty}$ as defined in (18), in terms of the odometer model for the action. We first give a basic fact, where recall that $\widehat{G}_{\ell}$ was defined in (17).

LEMMA 3.11. $\mathcal{D}_{x}=\bigcap_{\ell>0} \widehat{G}_{\ell}$.

Proof. For $\widehat{h} \in \mathcal{D}_{x}$, by (18) we have $\widehat{h}=\left(h_{i}\right)$ where $h_{i} \in G_{i}$ for all $i \geq 1$ and $h_{i+1}^{-1} h_{i} \in G_{i}$. Since $G_{i+1} \subset G_{i}$ for all $i \geq 1$, given any $\ell \geq 1$ this implies that we can choose the sequence $\left(h_{i}\right)$ so that $h_{i}=h_{\ell} \in G_{\ell}$ for all $i \leq \ell$. Thus, if $\widehat{h} \in \mathcal{D}_{x}$ then $\widehat{h} \in \widehat{G}_{\ell}$, for all $\ell \geq 1$, and so $\mathcal{D}_{x} \subset \cap_{\ell>0} \widehat{G}_{\ell}$.

Conversely, suppose that $\widehat{h} \in \widehat{G}_{\ell}$ for all $\ell \geq 0$, and hence $\widehat{h} \cdot \widehat{G}_{\ell}=\widehat{G}_{\ell}$. Let $\widehat{U}_{\ell} \equiv \widehat{G}_{\ell} / \mathcal{D}_{x} \subset \widehat{G}_{\infty} / \mathcal{D}_{x}$. Then we have $\Theta_{x}\left(U_{\ell}\right)=\widehat{U}_{\ell}$, so that $\widehat{U}_{\ell}$ is identified with the clopen neighborhood $U_{\ell} \subset \mathfrak{X}$ of $x$. Since $\cap_{\ell>0} U_{\ell}=\{x\}$, it follows that $\widehat{h} \cdot x=x$, and so $\widehat{h} \in \mathcal{D}_{x}$. Thus, $\mathcal{D}_{x}=\cap_{\ell>0} \widehat{G}_{\ell}$.

Now recall that in Section 2.3, for adapted sets $V \subset U$, we defined in (8) the groups $\widehat{H}_{U, V}$ and $\widehat{K}_{U, V}$. Their analogs for the odometer model of the action are the groups

$$
\begin{aligned}
\widehat{H}_{X_{\infty}, \widehat{U}_{\ell}} & \equiv\left\{\widehat{g} \in \widehat{G}_{\infty} \mid \widehat{\Phi}_{x}(\widehat{g})\left(\widehat{U}_{\ell}\right)=\widehat{U}_{\ell}\right\}=\widehat{G}_{\ell} \\
\widehat{K}_{X_{\infty}, \widehat{U}_{\ell}} & \equiv\left\{\widehat{g} \in \widehat{G}_{\ell} \mid \widehat{\Phi}_{x}(\widehat{g}) \text { acts as the identity on } \widehat{U}_{\ell}\right\} \subset \mathcal{D}_{x} .
\end{aligned}
$$

For each $\ell \geq 0$, define

$$
K_{\ell} \equiv \widehat{K}_{X_{\infty}, \widehat{U}_{\ell}} .
$$

Then $\widehat{U}_{\ell+1} \subset \widehat{U}_{\ell}$ implies that $K_{\ell} \subset K_{\ell+1}$ for all $\ell \geq 0$. Thus, we obtain an increasing chain $\mathcal{K}(\mathcal{U})=\left\{K_{0} \subset K_{1} \subset K_{2} \subset \cdots\right\}$ of subgroups of $\mathcal{D}_{x}$. This is called the stabilizer group chain.

Next, Lemma 3.11 implies that for each $\ell \geq 0$, the adjoint action of $\widehat{h} \in \mathcal{D}_{x}$ restricts to a map $\operatorname{Ad}_{\ell}(\widehat{h}): \widehat{G}_{\ell} \rightarrow \widehat{G}_{\ell}$. Define

$$
Z_{\ell} \equiv \operatorname{ker}\left\{\operatorname{Ad}_{\ell}: \mathcal{D}_{x} \rightarrow \operatorname{Homeo}\left(\widehat{G}_{\ell}\right)\right\} \subset \mathcal{D}_{x} .
$$

Then $\widehat{G}_{\ell+1} \subset \widehat{G}_{\ell}$ implies that $Z_{\ell} \subset Z_{\ell+1}$ for all $\ell \geq 0$. Thus, we obtain another increasing chain $\mathcal{Z}(\mathcal{U})=\left\{Z_{0} \subset Z_{1} \subset Z_{2} \subset \cdots\right\}$ of subgroups of $\mathcal{D}_{x}$. This is called the centralizer group chain, due to the following observation. For a group $H$ and subgroup $K \subset H$, define the centralizer of $K$ in $H$,

$$
\operatorname{Cen}(H, K)=\{h \in H \mid h k=k h \text {, for all } k \in K\} \subset H \text {. }
$$

Recall that $q_{\infty}: G \rightarrow \widehat{G}_{\infty}$ has dense image. 
LEMMA 3.12. $Z_{\ell}=\mathcal{D}_{x} \cap \operatorname{Cen}\left(\widehat{G}_{\infty}, \widehat{G}_{\ell}\right)=\mathcal{D}_{x} \cap \operatorname{Cen}\left(\widehat{G}_{\infty}, q_{\infty}\left(G_{\ell}\right)\right)$.

Proof. The equality $Z_{\ell}=\mathcal{D}_{x} \cap \operatorname{Cen}\left(\widehat{G}_{\infty}, \widehat{G}_{\ell}\right)$ is just a restatement of (23). The second equality follows from the observation that if $\widehat{h} \in \widehat{G}_{\infty}$ commutes with the image group $q_{\infty}\left(G_{\ell}\right)$, then it also commutes with every element in its closure $\widehat{G}_{\ell}$, hence $\operatorname{Cen}\left(\widehat{G}_{\infty}, q_{\infty}\left(G_{\ell}\right)\right) \subset \operatorname{Cen}\left(\widehat{G}_{\infty}, \widehat{G}_{\ell}\right)$.

The two group chains $\mathcal{Z}(\mathcal{U})$ and $\mathcal{K}(\mathcal{U})$ are closely related.

LEMMA 3.13. There is an inclusion of group chains $\iota_{\infty}: \mathcal{Z}(\mathcal{U}) \rightarrow \mathcal{K}(\mathcal{U})$.

Proof. By the definition (23), for each $\ell \geq 0$ and $\widehat{h} \in Z_{\ell}$, the action $\operatorname{Ad}_{\ell}(\widehat{h})$ on $\widehat{G}_{\ell}$ is trivial. Hence by Lemma 2.7, the induced action $\widehat{\Phi}_{x}(\widehat{h})$ on $\widehat{U}_{\ell} \equiv \widehat{G}_{\ell} / \mathcal{D}_{x}$ is trivial. Thus, there is an inclusion map $\iota_{\ell}: Z_{\ell} \subset K_{\ell}$, which yields the inclusion map $\iota_{\infty}=\left(\iota_{\ell}\right)$ on group chains.

Consider $\widehat{h} \in Z_{\ell+1}$ such that $\widehat{h} \notin Z_{\ell}$. Then $\widehat{\Phi}_{x}(\widehat{h})$ acts trivially on $\widehat{U}_{\ell+1}$, and $\operatorname{Ad}_{\ell}(\widehat{h})$ acts nontrivially on $\widehat{G}_{\ell}$. However, it is possible that $\widehat{\Phi}_{x}(\widehat{h})$ also acts trivially on $\widehat{U}_{\ell}$, while the adjoint action $\mathbf{A d}_{\ell}(\widehat{h})$ acts non-trivially on the subgroup $\mathcal{D}_{x}$ as discussed in Remark 2.8. The point is that comparing the subgroups $Z_{\ell} \subset K_{\ell}$ involves algebraic properties of the inclusion $\mathcal{D}_{x} \subset \widehat{G}_{\infty}$.

Finally, given $\widehat{h} \in \mathcal{D}_{x}$ which is represented by a sequence $\widehat{h}=\left(h_{\ell}\right)$ with $h_{\ell}=h \in G$ for all $\ell \geq 0$, then $h \in \widehat{G}_{\ell}$ of all $\ell \geq 0$, so $h \in K\left(\mathcal{G}_{\mathcal{U}}^{x}\right)$. Conversely, recall that $N K\left(\mathcal{G}_{\mathcal{U}}^{x}\right)$ is the largest normal subgroup of $K\left(\mathcal{G}_{\mathcal{U}}^{x}\right)$, and so $N K\left(\mathcal{G}_{\mathcal{U}}^{x}\right) \subset C_{\ell}$ for all $\ell \geq 1$, hence each $h \in N K\left(\mathcal{G}_{\mathcal{U}}^{x}\right)$ acts trivially on $\widehat{G}_{\infty}$. Define the quotient group

$$
\mathcal{D}_{x}^{G} \equiv K\left(\mathcal{G}_{\mathcal{U}}^{x}\right) / N K\left(\mathcal{G}_{\mathcal{U}}^{x}\right) \subset \mathcal{D}_{x}
$$

then each $h \in \mathcal{D}_{x}^{G}$ is represented by a constant sequence $(h)$ for $h \in G$. One can think of the elements of $\mathcal{D}_{x}^{G}$ as the "G-rational points" of $\mathcal{D}_{x}$. Of course, if $K\left(\mathcal{G}_{\mathcal{U}}^{x}\right)$ is trivial, then there are no such points. The examples in Section 8 illustrate actions with non-trivial $G$-rational points.

\section{Direct Limit COnjugacy invariants}

In this section, we use the direct limit construction for the group chains $\mathcal{Z}(\mathcal{U})$ and $\mathcal{K}(\mathcal{U})$ to obtain conjugacy invariants of a Cantor action $(\mathfrak{X}, G, \Phi)$. These are subtle invariants of an action, and each is independent of the other, as shown by Theorem 7.5 and the examples in Section 8 . We first briefly recall the basic formulation of direct limits, and then apply these ideas to obtain the stabilizer limit group $\Upsilon_{s}(\Phi)$ and the centralizer limit group $\Upsilon_{c}(\Phi)$ for a Cantor action $(\mathfrak{X}, G, \Phi)$.

4.1. Direct limits. We give the construction and properties of the direct limit in the category of groups. Basic references for this standard concept are Eilenberg and Steenrod 20, Chapter VIII, Section 2], and the text by Munkres [43, Section 73], which give proofs of the following results.

DEFINITION 4.1. A directed system of groups $\mathcal{G}\left(G_{\lambda}, \phi_{\lambda}^{\lambda^{\prime}}, \Lambda\right)$ over a directed set $\Lambda$ is collection of groups $\left\{G_{\lambda} \mid \lambda \in \Lambda\right\}$, and for each $\lambda, \lambda^{\prime} \in \Lambda$ with $\lambda<\lambda^{\prime}$, a group homomorphism $\phi_{\lambda}^{\lambda^{\prime}}: G_{\lambda} \rightarrow G_{\lambda^{\prime}}$. We require that $\phi_{\lambda}^{\lambda}=i d_{G_{\lambda}}$, and for $\lambda<\lambda^{\prime}<\lambda^{\prime \prime}$ that $\phi_{\lambda}^{\lambda^{\prime \prime}}=\phi_{\lambda^{\prime}}^{\lambda^{\prime \prime}} \circ \phi_{\lambda}^{\lambda^{\prime}}$.

DEFINITION 4.2. Let $\mathcal{G}\left(G_{\lambda}, \phi_{\lambda}^{\lambda^{\prime}}, \Lambda\right)$ be a directed system of groups over a directed set $\Lambda$. Define an equivalence relation on the disjoint union $\bigcup_{\lambda \in \Lambda} G_{\lambda}$ where for $g_{\lambda} \in G_{\lambda}$ and $g_{\lambda^{\prime}} \in G_{\lambda^{\prime}}$, we set $g_{\lambda} \sim g_{\lambda^{\prime}}$ if for all $\lambda^{\prime \prime} \in \Lambda$ with $\lambda<\lambda^{\prime \prime}$ and $\lambda^{\prime}<\lambda^{\prime \prime}$, then $\phi_{\lambda}^{\lambda^{\prime \prime}}\left(g_{\lambda}\right)=\phi_{\lambda^{\prime}}^{\lambda^{\prime \prime}}\left(g_{\lambda^{\prime}}\right)$.

DEFINITION 4.3. The direct limit of a directed system of groups $\mathcal{G}\left(G_{\lambda}, \phi_{\lambda}^{\lambda^{\prime}}, \Lambda\right)$ over a directed set $\Lambda$ is defined to be the set of equivalence classes

$$
\underset{\lim _{\longrightarrow}}{\longrightarrow} \mathcal{G}\left(G_{\lambda}, \phi_{\lambda}^{\lambda^{\prime}}, \Lambda\right) \equiv \bigcup_{\lambda \in \Lambda} G_{\lambda} / g_{\lambda} \sim g_{\lambda^{\prime}}
$$


The group structure on $\lim _{\longrightarrow} \mathcal{G}\left(G_{\lambda}, \phi_{\lambda}^{\lambda^{\prime}}, \Lambda\right)$ is inherited from that on the summands. For $\lambda \in \Lambda$ and $g_{\lambda} \in G_{\lambda}$ let $\left[g_{\lambda}\right] \in \underset{\lim }{\longrightarrow}\left(G_{\lambda}, \phi_{\lambda}^{\lambda^{\prime}}, \Lambda\right)$ denote the equivalence class it determines.

DEFINITION 4.4. A map $\Xi$ between directed systems of groups $\mathcal{G}\left(G_{\lambda}, \phi_{\lambda}^{\lambda^{\prime}}, \Lambda\right)$ and $\mathcal{G}\left(H_{\omega}, \psi_{\omega}^{\omega^{\prime}}, \Omega\right)$ is an order-preserving map $\xi: \Lambda \rightarrow \Omega$, and for each $\lambda \in \Lambda$ a group homomorphism $\xi_{\lambda}: G_{\lambda} \rightarrow H_{\xi(\lambda)}$ such that for $\lambda<\lambda^{\prime}$ we have

$$
\xi_{\lambda^{\prime}} \circ \phi_{\lambda}^{\lambda^{\prime}}=\phi_{\xi(\lambda)}^{\xi\left(\lambda^{\prime}\right)} \circ \xi_{\lambda}: G_{\lambda} \rightarrow H_{\xi\left(\lambda^{\prime}\right)} .
$$

PROPOSITION 4.5. A map $\Xi$ between directed systems of groups $\mathcal{G}\left(G_{\lambda}, \phi_{\lambda}^{\lambda^{\prime}}, \Lambda\right)$ and $\mathcal{G}\left(H_{\omega}, \psi_{\omega}^{\omega^{\prime}}, \Omega\right)$ induces a homomorphism $\Xi: \lim _{\longrightarrow} \mathcal{G}\left(G_{\lambda}, \phi_{\lambda}^{\lambda^{\prime}}, \Lambda\right) \longrightarrow \lim _{\longrightarrow} \mathcal{G}\left(H_{\omega}, \psi_{\omega}^{\omega^{\prime}}, \Omega\right)$.

We next recall some special cases of directed systems of groups and maps between directed systems of groups. We single out two special classes of maps.

PROPOSITION 4.6. A map $\Xi$ between directed systems of groups $\mathcal{G}\left(G_{\lambda}, \phi_{\lambda}^{\lambda^{\prime}}, \Lambda\right)$ and $\mathcal{G}\left(H_{\omega}, \psi_{\omega}^{\omega^{\prime}}, \Omega\right)$ is a monomorphism if each of the maps $\xi_{\lambda}: G_{\lambda} \rightarrow H_{\xi(\lambda)}$ for $\lambda \in \Lambda$ is a monomorphism of groups. Then the induced map $\Xi: \lim _{\longrightarrow} \mathcal{G}\left(G_{\lambda}, \phi_{\lambda}^{\lambda^{\prime}}, \Lambda\right) \longrightarrow \underline{\lim } \mathcal{G}\left(H_{\omega}, \psi_{\omega}^{\omega^{\prime}}, \Omega\right)$ is a group monomorphism.

PROPOSITION 4.7. A map $\Xi$ between directed systems of groups $\mathcal{G}\left(G_{\lambda}, \phi_{\lambda}^{\lambda^{\prime}}, \Lambda\right)$ and $\mathcal{G}\left(H_{\omega}, \psi_{\omega}^{\omega^{\prime}}, \Omega\right)$ is an isomorphism if each of the maps $\xi_{\lambda}: G_{\lambda} \rightarrow H_{\xi(\lambda)}$ for $\lambda \in \Lambda$ is an isomorphism of groups. Then the induced map $\rightrightarrows: \lim _{\longrightarrow} \mathcal{G}\left(G_{\lambda}, \phi_{\lambda}^{\lambda^{\prime}}, \Lambda\right) \longrightarrow \underset{\lim }{\longrightarrow} \mathcal{G}\left(H_{\omega}, \psi_{\omega}^{\omega^{\prime}}, \Omega\right)$ is a group isomorphism.

A subset $\Lambda_{0} \subset \Lambda$ of a directed set is said to be cofinal if for each $\lambda \in \Lambda$, there exists $\lambda^{\prime} \in \Lambda_{0}$ with $\lambda<\lambda^{\prime}$. Then we have

PROPOSITION 4.8. Let $\mathcal{G}\left(G_{\lambda}, \phi_{\lambda}^{\lambda^{\prime}}, \Lambda\right)$ be a directed systems of groups, and $\Lambda_{0} \subset \Lambda$ be a cofinal set. Then the inclusion induces a group isomorphism $\lim _{\longrightarrow} \mathcal{G}\left(G_{\lambda}, \phi_{\lambda}^{\lambda^{\prime}}, \Lambda_{0}\right) \cong \lim _{\longrightarrow} \mathcal{G}\left(G_{\lambda}, \phi_{\lambda}^{\lambda^{\prime}}, \Lambda\right)$.

In addition, we introduce two special classes of direct limit systems.

DEFINITION 4.9. A directed system of groups $\mathcal{G}\left(G_{\lambda}, \phi_{\lambda}^{\lambda^{\prime}}, \Lambda\right)$ is said to be bounded if there exists $\lambda_{0} \in \Lambda$ such that for all $\lambda_{0}<\lambda<\lambda^{\prime}$ the map $\phi_{\lambda}^{\lambda^{\prime}}$ is a group isomorphism. The directed system is said to be unbounded if no such $\lambda_{0}$ exists.

DEFINITION 4.10. A directed system $\mathcal{G}\left(G_{\lambda}, \phi_{\lambda}^{\lambda^{\prime}}, \Lambda\right)$ has finite type if each group $G_{\lambda}$ is finite.

For our purposes, the ordered sets $\Lambda$ and $\Omega$ above will always be assumed to be a subset of the non-negative integers $\mathbb{N}$ with the natural order. All maps $\left\{\phi_{\lambda}^{\lambda^{\prime}} \mid \lambda<\lambda^{\prime} \in \Lambda\right\}$ in our applications will be group inclusions, possibly isomorphisms. For a morphism $\Xi$ of directed systems of groups, the maps $\{\xi(\lambda) \mid \lambda \in \Lambda\}$ will be be group inclusions (possibly isomorphisms).

Now assume that $\Lambda \subset \mathbb{N}$, then $\underset{\lim }{\longrightarrow} \mathcal{G}\left(G_{\lambda}, \phi_{\lambda}^{\lambda^{\prime}}, \Lambda\right)$ inherits a filtration from its definition.

DEFINITION 4.11. The height function $\lambda: \lim \mathcal{G}\left(G_{\lambda}, \phi_{\lambda}^{\lambda^{\prime}}, \Lambda\right) \rightarrow \Lambda$ is defined for an equivalence class $[g]$ to be the least $\lambda$ such that $[g]=\left[g_{\lambda}\right]$ for some $g_{\lambda} \in G_{\lambda}$.

REMARK 4.12. Note that while the height function need not be preserved by a map between directed systems, it does yield a well-defined order, where $\left[g_{1}\right] \leq_{\lambda}\left[g_{2}\right]$ if $\lambda\left(g_{1}\right)<\lambda\left(g_{2}\right)$.

REMARK 4.13. Suppose there exists a group $\mathfrak{G}$ such that $G_{\lambda} \subset \mathfrak{G}$ for all $\lambda \in \Lambda$, and the maps $\phi_{\lambda}^{\lambda^{\prime}}$ are inclusions of subgroups. Define

$$
G_{\infty} \equiv \bigcup_{\lambda \in \Lambda} G_{\lambda}
$$

For $g_{\lambda} \in G_{\lambda}$ and $g_{\lambda^{\prime}}^{\prime} \in G_{\lambda^{\prime}}$ then there exists $\lambda^{\prime \prime}>\max \left\{\lambda, \lambda^{\prime}\right\}$ so that we can consider $g_{\lambda}, g_{\lambda^{\prime}} \in G_{\lambda^{\prime \prime}}$ and then $g_{\lambda}+g_{\lambda^{\prime}}^{\prime}$ is defined in $G_{\lambda^{\prime \prime}} \subset G_{\infty}$. That is, $G_{\infty}$ is a subgroup of $\mathfrak{G}$. 
Define a height function $\lambda_{\infty}: G_{\infty} \rightarrow \Lambda$ by $\lambda_{\infty}(g) \leq \lambda$ if $g \in G_{\lambda}$. Given the pair $\left\{G_{\infty}, \lambda_{\infty}\right\}$, define $\widetilde{G}_{\lambda} \equiv\left\{g \in G_{\infty} \mid \lambda_{\infty}(g) \leq \lambda\right\}$, and introduce maps given by inclusions. Let $\mathcal{G}\left(G_{\infty}, \lambda_{\infty}\right)$ denote the resulting direct limit of groups, which is a small abuse of notation. It is then immediate that there is an isomorphism of direct limits $\mathcal{G}\left(G_{\lambda}, \phi_{\lambda}^{\lambda^{\prime}}, \Lambda\right) \cong \mathcal{G}\left(G_{\infty}, \lambda_{\infty}\right)$. Thus, one can view a direct limit of subgroups of $\mathfrak{G}$ as a subgroup $G_{\infty} \subset \mathfrak{G}$, equipped with a height function $\lambda_{\infty}: G_{\infty} \rightarrow \Lambda$.

In the case when the directed system of groups $\mathcal{G}\left(G_{\lambda}, \phi_{\lambda}^{\lambda^{\prime}}, \Lambda\right)$ is bounded, with $\lambda_{0}$ an upper bound, then the inclusion of the constant sequence $\mathcal{G}\left(G_{\lambda_{0}}, I d_{G_{\lambda_{0}}},\left\{\lambda_{0}\right\}\right) \subset \mathcal{G}\left(G_{\lambda}, \phi_{\lambda}^{\lambda^{\prime}}, \Lambda\right)$ is an isomorphism, and we have $G_{\infty}=G_{\lambda_{0}}$. In other words, the direct limit of a bounded direct limit collapses.

4.2. The stabilizer and centralizer limit groups. We now show that the chain of stabilizer subgroups defined by (22), and the chain of centralizer subgroups defined in Lemma [3.12, yield direct limit groups whose isomorphism classes are conjugacy invariants of Cantor actions.

DEFINITION 4.14. Let $(\mathfrak{X}, G, \Phi)$ be a Cantor action, and $\mathcal{G}_{\mathcal{U}}^{x}=\left\{G_{\ell} \mid \ell \geq 0\right\}$ be the group chain associated to an adapted neighborhood basis $\mathcal{U}$ at $x \in \mathfrak{X}$. Let $\left\{K_{\ell} \mid \ell \geq 0\right\}$ be the chain of stabilizer groups associated to $\mathcal{G}_{\mathcal{U}}^{x}$, and let $\left\{Z_{\ell} \mid \ell \geq 0\right\}$ be the chain of centralizer groups associated to $\mathcal{G}_{\mathcal{U}}^{x}$. Then define:

(1) The stabilizer (direct limit) group is $\Upsilon_{s}^{x}(\Phi)=\lim _{\longrightarrow} \mathcal{G}\left(K_{\ell}, \iota_{\ell}^{\ell^{\prime}}, \mathbb{N}\right)$;

(2) The centralizer (direct limit) group is $\Upsilon_{c}^{x}(\Phi)=\underset{\lim }{\longrightarrow}\left(Z_{\ell}, \iota_{\ell}^{\ell^{\prime}}, \mathbb{N}\right)$;

where $\iota_{\ell}^{\ell^{\prime}}$ denotes the inclusion map, for $0 \leq \ell \leq \ell^{\prime}$.

We now come to the main result of this section.

THEOREM 4.15. Let $(\mathfrak{X}, G, \Phi)$ be a Cantor action. The direct limit isomorphism classes of $\Upsilon_{s}^{x}(\Phi)$ and $\Upsilon_{c}^{x}(\Phi)$ are invariants of the conjugacy class of the action $\Phi$. These isomorphism classes are denoted by $\Upsilon_{s}(\Phi)$ and $\Upsilon_{c}(\Phi)$, respectively, where we suppress the basepoint $x$ in the notation.

Proof. Let $(\mathfrak{X}, G, \Phi)$ and $\left(\mathfrak{X}^{\prime}, G, \Psi\right)$ be conjugate Cantor actions by a homeomorphism $h: \mathfrak{X}^{\prime} \rightarrow \mathfrak{X}$. Let $\mathcal{U}=\left\{U_{\ell} \subset \mathfrak{X} \mid \ell \geq 0\right\}$ be an adapted neighborhood basis at $x \in \mathfrak{X}$ for the action $\Phi$, and let $\mathcal{V}=\left\{V_{\ell} \subset \mathfrak{X}^{\prime} \mid \ell \geq 0\right\}$ be an adapted neighborhood basis at $z \in \mathfrak{X}^{\prime}$ for the action $\Psi$. Then $\mathcal{U}^{\prime}=\left\{U_{\ell}^{\prime}=h\left(V_{\ell}\right) \subset \mathfrak{X} \mid \ell \geq 0\right\}$ is an adapted neighborhood basis at $y=h(z)$ for the action $\Phi$, and the group chain in $G$ associated to $\mathcal{V}$ and the action $\Psi$ coincides with the group chain in $G$ associated to $\mathcal{U}^{\prime}$ and the action $\Phi$. Thus, by Theorem 3.7. we need to show that the direct limits $\Upsilon_{s}^{x}(\Phi)$ and $\Upsilon_{c}^{x}(\Phi)$, associated to the group chain $\mathcal{G}_{\mathcal{U}}^{x}$ in $G$, are isomorphic as direct limits to the direct limits $\Upsilon_{s}^{y}(\Phi)$ and $\Upsilon_{c}^{y}(\Phi)$ associated to the group chain $\mathcal{G}_{\mathcal{U}^{\prime}}^{y}$.

First consider the case where $x=y$, so we are given two adapted neighborhood bases at a common basepoint $x, \mathcal{U}=\left\{U_{\ell} \subset \mathfrak{X} \mid \ell \geq 0\right\}$ and $\mathcal{U}^{\prime}=\left\{U_{\ell}^{\prime} \subset \mathfrak{X} \mid \ell \geq 0\right\}$, with corresponding group chains $\mathcal{G}_{\mathcal{U}}^{x}=\left\{G_{\ell} \mid \ell \geq 0\right\}$ and $\mathcal{G}_{\mathcal{U}^{\prime}}^{x}=\left\{G_{\ell}^{\prime} \mid \ell \geq 0\right\}$.

As $\mathcal{U}$ and $\mathcal{U}^{\prime}$ are both adapted neighborhood basis at $x$, there exists increasing sequences of indices $1 \leq i_{1}<i_{2}<i_{3}<\cdots$ and $1 \leq j_{1}<j_{2}<j_{3}<\cdots$ such that we have a descending sequence of adapted clopen sets at $x$, where $i_{0}=j_{0}=0$,

$$
\mathfrak{X}=U_{0}=U_{0}^{\prime} \supset U_{i_{1}} \supset U_{j_{1}}^{\prime} \supset U_{i_{2}} \supset U_{j_{2}}^{\prime} \supset \cdots .
$$

Then by Theorem 3.7 and passing to a subsequence, we can assume without loss of generality that $i_{\ell}=\ell$ and $j_{\ell}=\ell$, for $\ell \geq 0$. Introduce a common refinement $\mathcal{U}^{\prime \prime}=\left\{U_{\ell}^{\prime \prime} \mid \ell \geq 0\right\}$ of these chains of clopen sets, where $U_{2 \ell}^{\prime \prime}=U_{\ell}$ and $U_{2 \ell-1}^{\prime \prime}=U_{\ell}^{\prime}$.

Let $\mathcal{G}_{\mathcal{U}^{\prime \prime}}^{x}=\left\{G_{\ell}^{\prime \prime} \mid \ell \geq 0\right\}$ be the group chain associated to $\mathcal{U}^{\prime \prime}$, then $G_{2 \ell}^{\prime \prime}=G_{\ell}$ and $G_{2 \ell-1}^{\prime \prime}=G_{\ell}^{\prime}$. Let $X_{\infty}, X_{\infty}^{\prime}$ and $X_{\infty}^{\prime \prime}$ denote the inverse limit spaces defined as in (13) by the group chains $\mathcal{G}_{\mathcal{U}}^{x}, \mathcal{G}_{\mathcal{U}^{\prime}}^{x}$ and $\mathcal{G}_{\mathcal{U}^{\prime \prime}}^{x}$, respectively. Let $\widehat{\mathcal{U}}=\left\{\widehat{U}_{\ell} \subset X_{\infty} \mid \ell \geq 0\right\}$ be the corresponding adapted basis at $X_{\infty}$, and likewise $\widehat{\mathcal{U}}^{\prime}=\left\{\widehat{U}_{\ell}^{\prime} \subset X_{\infty}^{\prime} \mid \ell \geq 0\right\}$ and $\widehat{\mathcal{U}}^{\prime \prime}=\left\{\widehat{U}_{\ell}^{\prime \prime} \subset X_{\infty}^{\prime \prime} \mid \ell \geq 0\right\}$ for $X_{\infty}^{\prime}$ and $X_{\infty}^{\prime \prime}$, respectively. 
By the discussion in Section 3.1, there are homeomorphisms $\Theta_{x}: \mathfrak{X} \rightarrow X_{\infty}, \Theta_{x}^{\prime}: \mathfrak{X} \rightarrow X_{\infty}^{\prime}$ and $\Theta_{x}^{\prime \prime}: \mathfrak{X} \rightarrow X_{\infty}^{\prime \prime}$ which intertwine the $G$-actions on these spaces. Introduce the basepoint preserving homeomorphisms $\tau=\Theta_{x} \circ\left(\Theta_{x}^{\prime \prime}\right)^{-1}: X_{\infty}^{\prime \prime} \rightarrow X_{\infty}$ and $\tau^{\prime}=\Theta_{x}^{\prime} \circ\left(\Theta_{x}^{\prime \prime}\right)^{-1}: X_{\infty}^{\prime \prime} \rightarrow X_{\infty}^{\prime}$. The maps $\tau$ and $\tau^{\prime}$ have simple descriptions in terms of sequences. Given $\left(g_{i}^{\prime \prime}\right) \in X_{\infty}^{\prime \prime}$ then $\tau\left(g_{i}^{\prime \prime}\right)=\left(g_{i}\right) \in X_{\infty}$ where $g_{i}=g_{2 i}^{\prime \prime}$, and $\tau^{\prime}\left(g_{i}^{\prime \prime}\right)=\left(g_{i}^{\prime}\right) \in X_{\infty}^{\prime}$ where $g_{i}^{\prime}=g_{2 i-1}^{\prime \prime}$.

Let $\widehat{G}_{\infty}, \widehat{G}_{\infty}^{\prime}$ and $\widehat{G}_{\infty}^{\prime \prime}$ denote the inverse limit groups defined as in (15) by the group chains $\mathcal{G}_{\mathcal{U}}^{x}, \mathcal{G}_{\mathcal{U}^{\prime}}^{x}$ and $\mathcal{G}_{\mathcal{U}^{\prime \prime}}^{x}$, respectively. By Theorem 3.2 , there are topological isomorphisms

$$
\widehat{\Theta}: \mathfrak{G}(\Phi) \rightarrow \widehat{G}_{\infty}, \widehat{\Theta}^{\prime}: \mathfrak{G}(\Phi) \rightarrow \widehat{G}_{\infty}^{\prime}, \widehat{\Theta}^{\prime \prime}: \mathfrak{G}(\Phi) \rightarrow \widehat{G}_{\infty}^{\prime \prime}
$$

There are topological isomorphisms $\widehat{\tau}=\widehat{\Theta} \circ\left(\widehat{\Theta}^{\prime \prime}\right)^{-1}: \widehat{G}_{\infty}^{\prime \prime} \rightarrow \widehat{G}_{\infty}$ and $\widehat{\tau}^{\prime}=\widehat{\Theta}^{\prime} \circ\left(\widehat{\Theta}^{\prime \prime}\right)^{-1}: \widehat{G}_{\infty}^{\prime \prime} \rightarrow \widehat{G}_{\infty}^{\prime}$. Given a sequence $\widehat{g}=\left(g_{i}^{\prime \prime}\right) \in \widehat{G}_{\infty}^{\prime \prime}$ then $\widehat{\tau}\left(g_{i}^{\prime \prime}\right)=\left(g_{i}=g_{2 i}^{\prime \prime}\right) \in \widehat{G}_{\infty}$ and $\widehat{\tau}^{\prime}\left(g_{i}^{\prime \prime}\right)=\left(g_{i}^{\prime}=g_{2 i-1}^{\prime \prime}\right) \in \widehat{G}_{\infty}^{\prime}$.

We first consider the case of the stabilizer group chains. Let $\mathcal{D}_{x}^{\prime \prime} \subset \widehat{G}_{\infty}^{\prime \prime}$ denote the discriminant for the chain $\mathcal{G}_{\mathcal{U}^{\prime \prime}}^{x}$, then the restriction $\widehat{\tau}: \mathcal{D}_{x}^{\prime \prime} \rightarrow \mathcal{D}_{x} \subset \widehat{G}_{\infty}$ is an isomorphism by Lemma 3.3 and Corollary 3.4, and likewise for $\widehat{\tau}^{\prime}: \mathcal{D}_{x}^{\prime \prime} \rightarrow \mathcal{D}_{x}^{\prime} \subset \widehat{G}_{\infty}^{\prime}$.

We show that the stabilizer groups associated to the chains $\mathcal{G}_{\mathcal{U}^{\prime \prime}}^{x}$ and $\mathcal{G}_{\mathcal{U}}^{x}$ are isomorphic, with the proof for $\mathcal{G}_{\mathcal{U}^{\prime \prime}}^{x}$ and $\mathcal{G}_{\mathcal{U}^{\prime}}^{x}$ being the same. For each $\ell \geq 0$, by (21) and (22) we have

$$
\begin{aligned}
K_{\ell} \equiv \widehat{K}_{X_{\infty}, \widehat{U}_{\ell}} & =\left\{\widehat{g} \in \widehat{G}_{\ell} \mid \widehat{\Phi}_{x}(\widehat{g}) \text { acts as the identity on } \widehat{U}_{\ell}\right\} \subset \mathcal{D}_{x} . \\
K_{\ell}^{\prime \prime} \equiv \widehat{K}_{X_{\infty}^{\prime \prime}, \widehat{U}_{\ell}^{\prime \prime}} & =\left\{\widehat{g} \in \widehat{G}_{\ell}^{\prime \prime} \mid \widehat{\Phi}_{x}^{\prime \prime}(\widehat{g}) \text { acts as the identity on } \widehat{U}_{\ell}^{\prime \prime}\right\} \subset \mathcal{D}_{x}^{\prime \prime} .
\end{aligned}
$$

The isomorphism $\widehat{\tau}: \widehat{G}_{2 \ell}^{\prime \prime} \rightarrow \widehat{G}_{\ell}$ induces a homeomorphism $\tau_{\ell}: \widehat{U}_{2 \ell}^{\prime \prime}=\widehat{G}_{2 \ell}^{\prime \prime} / \mathcal{D}_{x}^{\prime \prime} \rightarrow \widehat{U}_{\ell}=\widehat{G}_{\ell} / \mathcal{D}_{x}$. Thus $\widehat{\tau}$ induces an isomorphism $\widehat{\tau}: K_{2 \ell}^{\prime \prime}=\widehat{K}_{X_{\infty}^{\prime \prime}, \widehat{U}_{2 \ell}^{\prime \prime}} \rightarrow \widehat{K}_{X_{\infty}, \widehat{U}_{\ell}}=K_{\ell}$.

For $0 \leq \ell \leq \ell^{\prime}$, let $\phi_{\ell}^{\ell^{\prime}}: K_{\ell} \subset K_{\ell^{\prime}}$ and $\psi_{\ell}^{\ell^{\prime}}: K_{\ell}^{\prime \prime} \subset K_{\ell^{\prime}}^{\prime \prime}$ denote the inclusion maps. By Propositions 4.7 and 4.8, we obtain an isomorphism of direct limits

$$
\underset{\widetilde{\tau}}{\rightarrow}: \lim _{\longrightarrow} \mathcal{G}\left(K_{\ell}^{\prime \prime}, \psi_{\ell}^{\ell^{\prime}}, \mathbb{N}\right) \rightarrow \lim _{\longrightarrow} \mathcal{G}\left(K_{\ell}, \phi_{\ell}^{\ell^{\prime}}, \mathbb{N}\right)
$$

as was to be shown. Hence, the direct limits $\lim _{\longrightarrow} \mathcal{G}\left(K_{\ell}, \phi_{\ell}^{\ell^{\prime}}, \mathbb{N}\right)$ and $\underset{\longrightarrow}{\longrightarrow} \mathcal{G}\left(K_{\ell}^{\prime}, \psi_{\ell}^{\ell^{\prime}}, \mathbb{N}\right)$ are isomorphic.

Next, consider the case of the centralizer group chains, given by

$$
Z_{\ell}=\operatorname{Cen}\left(\widehat{G}_{\infty}, \iota_{\infty}\left(G_{\ell}\right)\right) \cap \mathcal{D}_{x}, Z_{\ell}^{\prime}=\operatorname{Cen}\left(\widehat{G}_{\infty}^{\prime}, \iota_{\infty}^{\prime}\left(G_{\ell}^{\prime}\right)\right) \cap \mathcal{D}_{x}^{\prime}, Z_{\ell}^{\prime \prime}=\operatorname{Cen}\left(\widehat{G}_{\infty}^{\prime \prime}, \iota_{\infty}\left(G_{\ell}^{\prime \prime}\right)\right) \cap \mathcal{D}_{x}^{\prime \prime},
$$

which define the group chains $\mathcal{Z}, \mathcal{Z}^{\prime}$ and $\mathcal{Z}^{\prime \prime}$, respectively.

Recall the topological isomorphisms $\widehat{\tau}: \widehat{G}_{\infty}^{\prime \prime} \rightarrow \widehat{G}_{\infty}$ and $\widehat{\tau}^{\prime}: \widehat{G}_{\infty}^{\prime \prime} \rightarrow \widehat{G}_{\infty}^{\prime}$ are given by $\widehat{\tau}\left(g_{i}\right)=\left(g_{2 i}\right)$ and $\widehat{\tau}^{\prime}\left(g_{i}\right)=\left(g_{2 i-1}\right)$, for $\widehat{g}=\left(g_{i}\right) \in \widehat{G}_{\infty}^{\prime \prime}$. Then $\widehat{\tau}$ induces isomorphisms $\widehat{\tau}_{\ell}: Z_{2 \ell}^{\prime \prime} \rightarrow Z_{\ell}$ and $\widehat{\tau}_{\ell}^{\prime}: Z_{2 \ell-1}^{\prime \prime} \rightarrow Z_{\ell}^{\prime}$. Thus, as for the case of the stabilizer group chains, we obtain an isomorphism

$$
\widehat{\widehat{\tau}}: \underset{\lim }{\longrightarrow} \mathcal{G}\left(Z_{\ell}^{\prime \prime}, \psi_{\ell}^{\ell^{\prime}}, \mathbb{N}\right) \rightarrow \underset{\lim }{\longrightarrow} \mathcal{G}\left(Z_{\ell}, \phi_{\ell}^{\ell^{\prime}}, \mathbb{N}\right),
$$

as was to be shown. Hence, the direct limits $\lim _{\longrightarrow} \mathcal{G}\left(Z_{\ell}, \phi_{\ell}^{\ell^{\prime}}, \mathbb{N}\right)$ and $\lim _{\longrightarrow} \mathcal{G}\left(Z_{\ell}^{\prime}, \phi_{\ell}^{\ell^{\prime}}, \mathbb{N}\right)$ are isomorphic.

Next, consider the case where $x$ and $y$ are distinct basepoints, and we are given adapted neighborhood bases $\mathcal{U}=\left\{U_{\ell} \subset \mathfrak{X} \mid \ell \geq 0\right\}$ at $x$ and $\mathcal{U}^{\prime}=\left\{U_{\ell}^{\prime} \subset \mathfrak{X} \mid \ell \geq 0\right\}$ at $y$, with corresponding group chains $\mathcal{G}_{\mathcal{U}}^{x}=\left\{G_{\ell} \mid \ell \geq 0\right\}$ and $\mathcal{G}_{\mathcal{U}^{\prime}}^{y}=\left\{G_{\ell}^{\prime} \mid \ell \geq 0\right\}$. Theorem 3.7 implies that the chains $\mathcal{G}_{\mathcal{U}}^{x}$ and $\mathcal{G}_{\mathcal{U}^{\prime}}^{y}$ are conjugate equivalent. That is, choose $\widehat{g}=\left(g_{i}\right) \in \mathfrak{G}(\Phi)$ such that $\widehat{g} \cdot y=x$, then the collection $\mathcal{U}^{\prime \prime}=\left\{g_{i} \cdot U_{\ell}^{\prime} \subset \mathfrak{X} \mid \ell \geq 0\right\}$ is an adapted neighborhood basis at $x$, and the associated group chain $\mathcal{G}_{\mathcal{U}^{\prime \prime}}^{x}=\left\{G_{\ell}^{\prime \prime}=g_{\ell}^{-1} \widehat{G}_{\ell}^{\prime} g_{\ell}\right\}$ is equivalent to $\mathcal{G}_{\mathcal{U}}^{x}$.

A key point is that the isomorphism between $\widehat{G}_{\ell}^{\prime} \subset \widehat{G}_{\infty}$ and $\widehat{G}_{\ell}^{\prime \prime} \subset \widehat{G}_{\infty}$ is induced by the conjugacy isomorphism $\operatorname{Ad}\left(g_{\ell}\right): G_{\ell} \rightarrow g_{\ell} G_{\ell} g_{\ell}^{-1}$ which also conjugates the stabilizer groups $K_{\ell}^{\prime \prime}$ and $K_{\ell}^{\prime}$, and likewise for the centralizer groups $Z_{\ell}^{\prime \prime}$ and $Z_{\ell}^{\prime}$. Then by Propositions 4.7 and 4.8 we obtain an isomorphism of direct limits. We are thus reduced to the case shown previously, where we are given direct limit groups obtained from adapted neighborhood systems centered at the same point $x$. 
REMARK 4.16. The proof of Theorem4.15 shows that the isomorphism between the direct limits is induced by a group isomorphism $\operatorname{Ad}(\widehat{g}): \widehat{G}_{\infty} \rightarrow \widehat{G}_{\infty}$. By Remark 4.13 , for $\mathfrak{G}=\widehat{G}_{\infty}$, the adjoint induces the isomorphism of subgroups $\operatorname{Ad}(\widehat{g}): K_{\infty} \cong K_{\infty}^{\prime}$ and $\operatorname{Ad}(\widehat{g}): Z_{\infty} \cong Z_{\infty}^{\prime}$. A key point is that this isomorphism preserves the height filtration on these groups as defined in Remark 4.13 ,

4.3. Properties of the stabilizer and centralizer groups. The direct limit groups $\Upsilon_{s}(\Phi)$ and $\Upsilon_{c}(\Phi)$ are invariants of Cantor actions whose dynamical implications will be considered in the following sections. We first give a restatement of Lemma 3.13 in the language of direct limits.

LEMMA 4.17. For $x \in \mathfrak{X}$, there is an inclusion of direct limit groups $\Upsilon_{c}(\Phi) \subset \Upsilon_{s}(\Phi)$.

Examples show that the inclusion $\Upsilon_{c}(\Phi) \subset \Upsilon_{s}(\Phi)$ can be proper. One source of this distinction is noted in Remark 2.8, that the adjoint map for $\mathcal{D}_{x}$ restricted to $\mathcal{D}_{x}$ need not be trivial, while the action on $U_{\ell}$ is trivial. We next introduce five classes of Cantor actions.

DEFINITION 4.18. A Cantor action $(\mathfrak{X}, G, \Phi)$ is said to be:

(1) stable if the stabilizer group $\Upsilon_{s}(\Phi)$ is bounded, and is said to be wild otherwise;

(2) algebraically stable if the its centralizer group $\Upsilon_{c}(\Phi)$ is bounded, and is said to be algebraically wild otherwise;

(3) wild of finite type if the stabilizer group $\Upsilon_{s}(\Phi)$ is unbounded, and represented by a chain of finite groups;

(4) wild of flat type if the stabilizer group $\Upsilon_{s}(\Phi)$ is unbounded, and $\Upsilon_{c}(\Phi)=\Upsilon_{s}(\Phi)$;

(5) dynamically wild if the stabilizer group $\Upsilon_{s}(\Phi)$ is unbounded, and is not of flat type.

Each of the properties in Definition 4.18 is a conjugacy invariant of the action by Theorem 4.15

The notion of wild Cantor actions was introduced by the authors in [35, Definition 4.6], as part of the study of the homeomorphism types of weak solenoids in their works [19, 35, 36. The definition of a wild action in [35] and in Definition 4.18 coincide. Moreover, the examples of wild actions constructed in [35, Section 8] are all of finite and flat type, while the examples of actions constructed in Section 8 below are dynamically wild and not of finite type. On the other hand, Corollary 1.7 of [36] implies that if $G$ is a finitely generated nilpotent group, then every Cantor action of $G$ must be both stable and algebraically stable.

\section{Analytic Regularity for stable Cantor aCtions}

In this section, we relate the bounded property of the stabilizer group $\Upsilon_{s}(\Phi)$ for a Cantor action $(\mathfrak{X}, G, \Phi)$ with "analytic regularity" of the action. We first recall some background context.

5.1. Locally quasi-analytic actions. Haefliger introduced in 34 the notion of a quasi-analytic topological action of a pseudogroup on a connected space $X$. Álvarez López and Candel in [1, Definition 9.4], and later Álvarez López and Moreira Galicia in [2, Definition 2.18], adapted the notion of a quasi-analytic topological action to the more general case where the action space $X$ need not be connected. The authors formulated in [35, 36] a notion of locally quasi-analytic Cantor actions, and showed the relation between this condition and the stable property for the action.

The quasi-analytic condition for a Cantor action is a modification of the notion of a topologically free action for general Cantor actions, which first appeared in the work of Boyle and Tomiyama [7] in their study of flip-conjugacy. Renault showed in [49, Section 3] that an action is topologically free if and only if the associated action groupoid is essentially principal. We first recall the definition of a topologically free action and some properties of this definition. Topological freeness and related ideas are discussed in more detail in [38, Section 2].

The isotropy group at $x \in \mathfrak{X}$ of an action $(\mathfrak{X}, G, \Phi)$ is $G_{x}=\{g \in G \mid g \cdot x=x\}$. A point $x \in \mathfrak{X}$ is said to have trivial isotropy if $G_{x}$ is the trivial group. All points in the orbit of $x$ then also have trivial isotropy, so form a dense subset of $\mathfrak{X}$ for a minimal action. 
Let $\operatorname{Fix}(g)=\{x \in \mathfrak{X} \mid \Phi(g) \cdot x=x\}$, then introduce the isotropy set

$$
\operatorname{Iso}(\mathfrak{X}, G, \Phi) \equiv\{x \in \mathfrak{X} \mid \exists g \in G, g \neq i d, g \cdot x=x\}=\bigcup_{e \neq g \in G} \operatorname{Fix}(g) .
$$

The action $(\mathfrak{X}, G, \Phi)$ is said to be topologically free if the set $\operatorname{Iso}(\mathfrak{X}, G, \Phi)$ is meager in $\mathfrak{X}$. Note that if $e \neq g \in G$ and $\Phi(g)$ acts trivially on $\mathfrak{X}$, then $\operatorname{Iso}(\mathfrak{X}, G, \Phi)=\mathfrak{X}$, and thus a topologically free action must be faithful. If the group $G$ is abelian, it is an exercise to show that a faithful minimal Cantor action $(\mathfrak{X}, G, \Phi)$ must be topologically free; see for example [36. Corollary 2.3].

We next restrict attention to Cantor actions, and use the special properties of equicontinuous actions to formulate local forms of the topologically free property. A Cantor action $(\mathfrak{X}, G, \Phi)$ is said to be locally topologically free if there exists $\varepsilon>0$ such that for any adapted set $U$ with $\operatorname{diam}(U)<\varepsilon$, the action of $G_{U}$ on $U$ is topologically free. There is another related notion, defined as follows:

DEFINITION 5.1. [1, Definition 9.4] A Cantor action $(\mathfrak{X}, G, \Phi)$ is locally quasi-analytic, or simply LQA, if there exists $\varepsilon>0$ such that for any adapted set $U \subset \mathfrak{X}$ with $\operatorname{diam}(U)<\varepsilon$, and for any adapted subset $V \subset U$, and elements $g_{1}, g_{2} \in G$

$$
\text { if the restrictions } \Phi\left(g_{1}\right)\left|V=\Phi\left(g_{2}\right)\right| V \text {, then } \Phi\left(g_{1}\right)\left|U=\Phi\left(g_{2}\right)\right| U \text {. }
$$

That is, by [36, Proposition 2.2], the action of $H_{U}=\Phi\left(G_{U}\right)$ on $U$ is topologically free.

If (32) holds for $U=\mathfrak{X}$, then the action of $\mathfrak{G}(\Phi)$ is topologically free.

Examples of equicontinuous Cantor actions $\mathfrak{X}$ which are locally quasi-analytic, but not quasi-analytic, are easily constructed, as given in [19, 35] for example. There is also a generalization of the locally quasi-analytic property for the action of the profinite closure group $\mathfrak{G}(\Phi)$.

DEFINITION 5.2. A Cantor action $(\mathfrak{X}, G, \Phi)$ is locally completely quasi-analytic, or simply LCQA, if there exists $\varepsilon>0$ such that for any adapted set $U \subset \mathfrak{X}$ with $\operatorname{diam}(U)<\varepsilon$, and for any adapted subset $V \subset U$, and elements $\widehat{h}_{1}, \widehat{h}_{2} \in \mathfrak{G}(\Phi)$

$$
\text { if the restrictions } \widehat{h}_{1}\left|V=\widehat{h}_{2}\right| V \text {, then } \widehat{h}_{1}\left|U=\widehat{h}_{2}\right| U \text {. }
$$

Equivalently, set $\widehat{h}=\widehat{h}_{1} \widehat{h}_{2}^{-1}$ then we have

$$
\text { if the restriction } \widehat{h}|V=i d| V \text {, then } \widehat{h}|U=i d| U \text {. }
$$

Since $\Phi(G) \subset \mathfrak{G}(\Phi)$, the LCQA property implies the LQA property for a Cantor action. It is not known however, if there exists a Cantor action $(\mathfrak{X}, G, \Phi)$ which is LQA but not LCQA. In particular, does there exists a free Cantor action which is not LCQA? All examples known to the authors which are not LCQA are also not LQA.

5.2. Bounded stabilizer groups. Recall from Definition 4.18 that a Cantor action $(\mathfrak{X}, G, \Phi)$ is stable if the stabilizer group $\Upsilon_{s}(\Phi)$ is bounded. Here is the main result of this section.

THEOREM 5.3. Let $(\mathfrak{X}, G, \Phi)$ be a Cantor action, then $\Phi$ is a locally completely quasi-analytic (LCQA) action if and only if its stabilizer limit group $\Upsilon_{s}(\Phi)$ is bounded.

Proof. Let $(\mathfrak{X}, G, \Phi)$ be a Cantor action, and $\mathcal{G}_{\mathcal{U}}^{x}=\left\{G_{\ell} \mid \ell \geq 0\right\}$ be the group chain associated to an adapted neighborhood basis $\mathcal{U}$ at $x \in \mathfrak{X}$. Let $\widehat{G}_{\infty}$ be defined as in (15), then by Theorem 3.2, there is a topological isomorphism $\widehat{\Theta}: \mathfrak{G}(\Phi) \rightarrow \widehat{G}_{\infty}$ which induces an isomorphism $\widehat{\Theta}: \mathfrak{G}(\Phi)_{x} \rightarrow \mathcal{D}_{x}$.

Let $\mathcal{K}(\mathcal{U})=\left\{K_{\ell} \subset \mathcal{D}_{x} \mid \ell \geq 0\right\}$ be the increasing chain of stabilizer subgroups defined in (22).

The map $\widehat{\Theta}$ induces a homeomorphism of $G$-spaces $\Theta_{x}: \mathfrak{G}(\Phi) / \mathfrak{G}(\Phi)_{x} \cong \mathfrak{X} \rightarrow \widehat{G}_{\infty} / \mathcal{D}_{x} \cong X_{\infty}$ by Corollary 3.4. Let $X_{\infty}$ have a metric such that $G$ acts on $X_{\infty}$ by isometries, then let $d_{\mathfrak{X}}$ be the metric on $\mathfrak{X}$ induced by the homeomorphism $\Theta_{x}$.

Recall that for $\ell \geq 0, \Theta_{x}$ identifies the clopen set $U_{\ell} \subset \mathfrak{X}$ with $\widehat{U}_{\ell} \equiv \widehat{G}_{\ell} / \mathcal{D}_{x} \subset \widehat{G}_{\infty} / \mathcal{D}_{x}$. 
Assume that $\Phi$ is LCQA, and let $\varepsilon>0$ be as in Definition 5.2. Let $\ell_{0} \geq 1$ be such that $\operatorname{diam}\left(U_{\ell}\right)<\varepsilon$ for all $\ell \geq \ell_{0}$. Thus, for $\ell>\ell_{0}$ the restricted action of $\widehat{G}_{\ell}$ on $\widehat{U}_{\ell}$ is quasi-analytic. Given $\widehat{h} \in \mathcal{D}_{x}$ suppose that $\widehat{h}$ acts trivially on $\widehat{U}_{\ell}$ then it must act trivially on $U_{\ell_{0}}$. That is, $K_{\ell} \subset K_{\ell_{0}}$. As the converse $K_{\ell_{0}} \subset K_{\ell}$ always holds, this implies that $\Upsilon_{s}^{x}(\Phi)$ is a bounded direct limit group.

Conversely, assume that $\Upsilon_{s}^{x}(\Phi)$ is a bounded direct limit group. Then there exists $\ell_{0}>0$ such that $K_{\ell_{0}}=K_{\ell}$ for all $\ell \geq \ell_{0}$. Let $\varepsilon_{0}>0$ be such that the ball of radius $\varepsilon_{0}$ about $x$ is contained in $U_{\ell_{0}}$.

Set $\varepsilon=\varepsilon_{0} / 2$. Let $U \subset \mathfrak{X}$ be an open set with with $\operatorname{diam}(U)<\varepsilon$, and let $V$ be an open subset with $V \subset U$. Then by minimality and equicontinuity of the action $\Phi$, there exists $g \in G$ such that $g \cdot x \in V$. By the choice of $\varepsilon$ we have that $U^{\prime}=g^{-1} \cdot U \subset U_{\ell_{0}}$. In addition, as $\mathcal{U}$ is a neighborhood basis at $x$, there exists $\ell>0$ such that $U_{\ell} \subset V^{\prime}=g^{-1} \cdot V$.

Let $\widehat{h} \in \mathfrak{G}(\Phi)$ such that $\widehat{h}$ acts as the identity on $V$, then $\widehat{h}^{\prime}=g^{-1} \widehat{h} \cdot g$ acts as the identity on $V^{\prime}$, and thus also on $U_{\ell}$ since $U_{\ell} \subset V^{\prime}$. In particular, $\widehat{h}^{\prime} \cdot x=x$, so $\widehat{h}^{\prime} \in \mathfrak{G}(\Phi)_{x}$ hence $\widehat{k}=\widehat{\Theta}\left(\widehat{h}^{\prime}\right) \in \mathcal{D}_{x}$. Moreover, $\widehat{k}$ acts as the identity on $\widehat{U}_{\ell}$ hence $\widehat{k} \in K_{\ell}$. We are given that $K_{\ell}=K_{\ell_{0}}$ so $\widehat{h}^{\prime} \in K_{\ell_{0}}$ hence acts as the identity on $\widehat{U}_{\ell_{0}}$. Thus, $\widehat{h}^{\prime}$ acts as the identity on $U^{\prime} \subset U_{\ell_{0}}$ and so $\widehat{h}$ acts as the identity on $U$, as was to be shown.

\section{ORBIT EQUIVALENCE AND RIGIDITY}

Let $(\mathfrak{X}, G, \Phi)$ and $\left(\mathfrak{X}^{\prime}, G, \Psi\right)$ be general Cantor actions. A homeomorphism $h: \mathfrak{X} \rightarrow \mathfrak{X}^{\prime}$ is a conjugacy between the two actions if $\Phi(g)=h^{-1} \circ \Psi(g) \circ h$ for all $g \in G$. A conjugacy map $h$ thus preserves the structure of the orbits as $G$-spaces, which is used in constructing conjugacy invariants for an action. For example, this was used in Theorem 4.15 to show that the isomorphism classes of the direct limit groups $\Upsilon_{s}(\Phi)$ and $\Upsilon_{c}(\Phi)$ are invariants of the conjugacy class of a Cantor action $(\mathfrak{X}, G, \Phi)$.

An orbit equivalence between two actions is a bijective map $h: \mathfrak{X} \rightarrow \mathfrak{X}^{\prime}$ which maps orbits of the action $\Phi$ to orbits of the action $\Psi$. In addition, one can impose additional assumptions on the map $h$, such as to assume that it is a measurable isomorphism with respect to given quasi-invariant measures on $\mathfrak{X}$ and $\mathfrak{X}^{\prime}$. In the measurable category of actions, this yields the notion of measurable orbit equivalence, a topic which has been extensively studied. A celebrated result by Connes, Feldman and Weiss in 11] showed that for essentially free actions of amenable groups, any two such actions are measurably orbit equivalent. For example, if $G$ is a nilpotent group, then any essentially free ergodic action of $G$ is measurably orbit equivalent to an ergodic $\mathbb{Z}$-action. Thus, a measurable orbit equivalence need not preserve the structure on orbits induced by the $G$-action. On the other hand, the results of Furman in [26] show that for measure preserving actions of higher rank lattice groups, measure orbit equivalence implies virtual conjugacy of the actions, hence the orbit structures are preserved in this case. For further discussion of properties of measurable orbit equivalence, see for example the survey by Gaboriau 27.

For general Cantor actions, continuous orbit equivalence is the more natural notion to study. One assumes that the orbit equivalence $h$ is a homeomorphism which, in addition, satisfies a "locally constant" property, as stated precisely in Definition 6.1. This notion was introduced by Boyle in his thesis [6], and see also [31], and has played a fundamental role in the classification of general Cantor actions in many subsequent works, as for example in [5, 28, 29]. The works of Renault [49] and $\mathrm{Li}$ [38, Theorem 1.2] use the notion of continuous orbit equivalence to classify the groupoid $C^{*}$-algebras associated to the action, in the case of topologically free actions. The related notion of the topological full group of an action has provided a rich source of examples of finitely generated groups with exceptional properties, as discussed for example in [15, 45].

The rigidity property for Cantor actions states that topological orbit equivalence implies virtual conjugacy of the actions. In essence, this is saying that an orbit equivalence which locally preserves the orbit structures, must preserve the orbit structures on a global scale. In this way, it can be seen as a property which is analogous to the measure rigidity property studied by Furman in [26]. The work of Cortez and Medynets in [13] and Li in [38] prove versions of topological rigidity. 
In this section, we study the consequences of continuous orbit equivalence between Cantor actions, without any assumption that the actions are topologically free. Our main result is Theorem 6.10. which considers the relation between the stabilizer groups for continuously orbit equivalent actions. We deduce that the property of being stable, as well as algebraically stable, is an invariant of continuous orbit equivalence.

We first recall in Section 6.1 some notions from the theory of orbit equivalence for Cantor actions. In Section 6.2 we discuss various notions of rigidity for Cantor actions. Then in Section 6.3 the rigidity results from [13, 38, are described. We then begin the proof of Theorem 6.10 in Section 6.3. and Section 2.3 contains some technical results needed to complete the proof. Theorem6.18 gives an extension of Theorem 3.3 by Cortez and Medynets in [13, and Theorem 1.5 in [36] by the authors.

6.1. Continuous orbit equivalence. Let $(\mathfrak{X}, G, \Phi)$ be a general Cantor action. Consider the equivalence relation on $\mathfrak{X}$ defined by the action,

$$
\mathcal{R}(\mathfrak{X}, G, \Phi) \equiv\{(x, g \cdot x)) \mid x \in \mathfrak{X}, g \in G\} \subset \mathfrak{X} \times \mathfrak{X} .
$$

Given actions $(\mathfrak{X}, G, \Phi)$ and $\left(\mathfrak{X}^{\prime}, H, \Psi\right)$, we say they are orbit equivalent if there exist a bijection $h: \mathfrak{X} \rightarrow \mathfrak{X}^{\prime}$ which maps $\mathcal{R}(\mathfrak{X}, G, \Phi)$ to $\mathcal{R}\left(\mathfrak{X}^{\prime}, H, \Psi\right)$, and similarly for the inverse map $h^{-1}$.

DEFINITION 6.1. Let $(\mathfrak{X}, G, \Phi)$ and $\left(\mathfrak{X}^{\prime}, H, \Psi\right)$ be general Cantor actions. A continuous orbit equivalence between the actions is a homeomorphism $h: \mathfrak{X} \rightarrow \mathfrak{X}^{\prime}$ which is an orbit equivalence, and satisfies the locally constant conditions:

(1) for each $x \in \mathfrak{X}$ and $g \in G$, there exists $\alpha(g, x) \in H$ and an open set $x \in U_{x} \subset \mathfrak{X}$ such that $\Psi(\alpha(g, x)) \circ h\left|U_{x}=h \circ \Phi(g)\right| U_{x} ;$

(2) for each $y \in \mathfrak{X}^{\prime}$ and $k \in H$, there exists $\beta(k, y) \in G$ and an open set $y \in V_{y} \subset \mathfrak{X}^{\prime}$ such that $\Phi(\beta(k, y)) \circ h\left|V_{y}=h \circ \Psi(k)\right| V_{y}$.

Note in particular that these conditions imply that the functions $\alpha: G \times \mathfrak{X} \rightarrow H$ and $\beta: H \times \mathfrak{X}^{\prime} \rightarrow G$ are continuous, as the groups $G$ and $H$ have the discrete topology.

One special class of examples of continuous orbit equivalences, are those for which the functions $\alpha(g, y)$ and $\beta(g, x)$ are constant in $y$. Then the identities (37) and (38) can be considered as defining "time-shifts" along the orbits, in analogy with the case when $G, H=\mathbb{Z}$.

Let $\left(\mathfrak{X}^{\prime}, H, \Psi\right)$ be a general Cantor action, let $h: \mathfrak{X} \rightarrow \mathfrak{X}^{\prime}$ be a homeomorphism which implements a continuous orbit equivalence between $(\mathfrak{X}, G, \Phi)$ and $\left(\mathfrak{X}^{\prime}, H, \Psi\right)$, and let

$$
\Psi^{h}: H \times \mathfrak{X} \rightarrow \mathfrak{X} \text {, where } \Psi^{h}(g)(x)=h^{-1} \circ \Psi(g) \circ h(x) \text {, for } g \in H .
$$

Then the orbits for the actions $\Psi^{h}$ and $\Phi$ are equal, so the identity map is an orbit equivalence between $\mathcal{R}(\mathfrak{X}, G, \Phi)$ and $\mathcal{R}\left(\mathfrak{X}, H, \Psi^{h}\right)$. Thus, for the study of orbit equivalence, it suffices to consider orbit equivalences for which $h$ is the identity map. Then the data of the orbit equivalence is encapsulated in the continuous, hence locally constant maps $\alpha: G \times \mathfrak{X} \rightarrow H$ and $\beta: H \times \mathfrak{X} \rightarrow G$. That is, for $y \in \mathfrak{X}$ and $g \in G$, there exist a clopen set $y \in U_{g, y} \subset \mathfrak{X}$ so that

$$
\Psi(\alpha(g, y)) \cdot z=\Phi(g) \cdot z \text { for } z \in U_{g, y},
$$

and for $k \in H$, there exists a clopen set $y \in V_{k, y} \subset \mathfrak{X}$ so that

$$
\Phi(\beta(k, y)) \cdot z=\Psi(k) \cdot z \text { for } z \in V_{k, y} .
$$

Next, recall the notion of a cocycle over an action.

DEFINITION 6.2. Let $\varphi: \Gamma \times X \rightarrow X$ be an action of the group $\Gamma$ on a space $X$. A map $c: \Gamma \times X \rightarrow \Gamma^{\prime}$ is a cocycle over the action if for all $\gamma, \gamma^{\prime} \in \Gamma$ and $x \in X$, we have

$$
c\left(\gamma^{\prime} \cdot \gamma, x\right)=c\left(\gamma^{\prime}, \varphi(\gamma)(x)\right) \cdot c(\gamma, x) .
$$


For an action which is free, or more generally is topologically free, then the identities (37) and (38) uniquely determine the values of $\alpha(g, y)$ and $\beta(k, y)$ for all $y \in \mathfrak{X}$ and $k \in G$, and thus the functions $\alpha$ and $\beta$ are cocycles over the actions $\Phi$ and $\Psi$, respectively. However, if the actions are not topologically free, then the identities (37) and (38) no longer uniquely specify the values of $\alpha(g, y)$ and $\beta(k, y)$, and so they need not satisfy the cocycle identity.

For continuous orbit equivalent topologically free actions whose orbit functions $\alpha$ and $\beta$ are constant, the cocycle identity (39) implies they define group homomorphisms. This leads to the notion of conjugacy of Cantor actions, as was studied by Li in [38, and also the related notion of structural conjugacy by Cortez and Medynets in [13. We use the following terminology:

DEFINITION 6.3. The Cantor actions $(\mathfrak{X}, G, \Phi)$ and $(\mathfrak{X}, H, \Psi)$ are said to be $\theta$-conjugate if there exists a group isomorphism $\theta: G \rightarrow H$ such that

$$
\Phi(g)(x)=\Psi^{\theta}(x) \equiv \Psi(\theta(g))(x) \quad \text {, for all } g \in G, x \in \mathfrak{X} .
$$

The identity (40) implies that we can choose the function $\alpha(g, x)=\theta(g)$ in Definition 6.1,

For the case where $G=H=\mathbb{Z}$, the involution $\theta(n)=-n$ is the only non-trivial isomorphism, and in this case $\theta$-conjugacy is the same as flip-conjugacy as studied by Boyle and Tamiyama 7 .

There is a related notion to Definition 6.3 where we assume that $\mathfrak{X}=\mathfrak{X}^{\prime}, G=H$, and $\theta$ is the identity. Define the center of the action:

$$
\operatorname{Aut}(\mathfrak{X}, G, \Phi)=\{h \in \operatorname{Homeo}(\mathfrak{X}) \mid h(\Phi(g) \cdot x)=\Phi(g) \cdot h(x) \text {, for all } x \in \mathfrak{X}, g \in G\} .
$$

Then we have the following relation between the direct limit group $\Upsilon_{c}^{x}(\Phi)$ and the center:

LEMMA 6.4. Let $(\mathfrak{X}, G, \Phi)$ be a Cantor action, and $\left\{G_{\ell} \mid \ell \geq 0\right\}$ be the group chain associated to an adapted neighborhood basis $\mathcal{U}$ at $x \in \mathfrak{X}$. Then for each $\ell \geq 1, Z_{\ell} \subset \operatorname{Aut}\left(\mathfrak{X}, G_{\ell}, \Phi\right)$.

Proof. Let $\widehat{h} \in Z_{\ell}$ which acts on $\widehat{G}_{\infty}$ by left multiplication. Then for any $\widehat{g} \in G_{\ell}$ we have $\widehat{g} \widehat{h}=\widehat{h} \widehat{g}$ by Lemma 3.12. Thus, for the isomorphism $\widehat{\Theta}: \mathfrak{G}(\Phi) \rightarrow \widehat{G}_{\infty}$ we have $h=\widehat{\Theta}^{-1}(\widehat{h}) \in \operatorname{Aut}\left(\mathfrak{X}, G_{\ell}, \Phi\right)$.

REMARK 6.5. Note that in the context of Lemma 6.4, while the action of $G_{\ell}$ on the clopen set $U_{\ell}$ is minimal, its action on $\mathfrak{X}$ cannot be minimal, as $U_{\ell}$ is adapted hence invariant by the action of $G_{\ell}$. Lemma 6.4 implies that when the action $\Phi$ is restricted to subactions by groups $G_{\ell}$, the groups $Z_{\ell}$ arise as the centers of these restricted actions. That is, while $\Phi$ may have trivial center, if the direct limit group $\Upsilon_{c}^{x}(\Phi)$ is non-trivial, then there exists restricted actions with non-trivial "symmetries".

6.2. Types of rigidity. Rigidity for a dynamical system can be viewed as asserting that two dynamical systems which are equivalent in some weaker sense, are also equivalent, possibly up to some finite indeterminacy, in some stronger sense. For Cantor systems, we formulate three notions of equivalence for their rigidity, each weaker than the previous one.

The first notion is based on $\theta$-conjugacy as given in Definition 6.3 .

DEFINITION 6.6. A Cantor action $(\mathfrak{X}, G, \Phi)$ is rigid if, given a continuous orbit equivalence $h: \mathfrak{X} \rightarrow \mathfrak{X}^{\prime}$ to a Cantor action $\left(\mathfrak{X}^{\prime}, G^{\prime}, \Psi\right)$, then $\Psi^{h}$ as defined by (36) is $\theta$-conjugate to $\Phi$.

This is the form of the rigidity property used by $\mathrm{Li} 38$.

It is elementary to construct examples of Cantor actions where $G$ is a cross-product of a normal subgroup by a finite quotient group, as in Examples A.2 and A.3 in [36, such that two actions are continuous orbit equivalent but are not $\theta$-conjugate actions. This is the situation considered by Cortez and Medynets in [13. They accordingly introduced the weaker notion of structural stability and proved their rigidity results for free actions in these terms. Their notion of structural stability coincides with what we call virtual rigidity below.

Let $U \subset \mathfrak{X}$ be adapted for the Cantor action $(\mathfrak{X}, G, \Phi)$. Let $\Phi_{U}: G_{U} \times U \rightarrow U$ denote the restricted action of $G_{U}$ on $U$. Similarly, for a Cantor action $\left(\mathfrak{X}^{\prime}, G^{\prime}, \Psi\right)$ with adapted set $V \subset \mathfrak{X}^{\prime}$, let $\left(V, G_{V}^{\prime}, \Psi_{V}\right)$ denote the restricted action. 
DEFINITION 6.7. Let $(\mathfrak{X}, G, \Phi)$ be a Cantor action. Then the action $(\mathfrak{X}, G, \Phi)$ is virtually rigid if, given a continuous orbit equivalence $h: \mathfrak{X} \rightarrow \mathfrak{X}^{\prime}$ to a Cantor action $\left(\mathfrak{X}^{\prime}, G^{\prime}, \Psi\right)$, there exists an adapted set $U \subset \mathfrak{X}$ for the action $\Phi$ such that $V=h(U) \subset \mathfrak{X}^{\prime}$ is adapted for the action $\Psi$, and there is an isomorphism $\theta_{U}: G_{U} \rightarrow G_{V}^{\prime}$ so that the action $\left(U, G_{U}, \Phi_{U}\right)$ is $\theta_{U}$-conjugate to $\left(V, G_{V}^{\prime}, \Psi_{V}^{h}\right)$.

Note that both Definitions 6.6 and 6.7 are essentially only applicable for topologically free actions on the full space $\mathfrak{X}$. The third notion we consider is return equivalence, which was used in the authors' work [35] for the study of the homeomorphism types of weak solenoids. For the geometric applications in [19, 35, the holonomy action on a transversal is the fundamental concept. Accordingly, return equivalence for Cantor actions is formulated in terms of the image group $H_{U}$ for an adapted subset $U \subset \mathfrak{X}$ as defined in (6).

Let $U \subset \mathfrak{X}$ be adapted for the Cantor action $(\mathfrak{X}, G, \Phi)$. Let $\bar{\Phi}_{U}: H_{U} \times U \rightarrow U$ denote the induced action of $H_{U}=\Phi\left(G_{U}\right) \subset \operatorname{Homeo}(U)$ on $U$. Similarly, for a Cantor action $\left(\mathfrak{X}^{\prime}, G^{\prime}, \Psi\right)$ with adapted set $V \subset \mathfrak{X}^{\prime}$, let $\left(V, H_{V}^{\prime}, \bar{\Psi}_{V}\right)$ denote the induced action.

DEFINITION 6.8. Two Cantor actions $(\mathfrak{X}, G, \Phi)$ and $\left(\mathfrak{X}^{\prime}, G^{\prime}, \Psi\right)$ are return equivalent if there exists an adapted set $U \subset \mathfrak{X}$ for the action $\Phi$, an adapted set $V \subset \mathfrak{X}^{\prime}$ for the action $\Psi$, and a homeomorphism $h_{U}: U \rightarrow V$ which induces a $\theta_{U}$-conjugacy between the action of $H_{U}$ on $U$ and the action of $H_{V}^{\prime}$ on $V$.

While the Definitions 6.7 and 6.8 are similar, the former requires an isomorphism between the subgroups $G_{U} \subset G$ and $G_{V}^{\prime} \subset G^{\prime}$, while the latter only requires an isomorphism between their respective image groups $H_{U}$ and $H_{V}^{\prime}$. Thus, Definition 6.8 is most relevant for the study of Cantor actions which are not topologically free.

For topologically free actions, an adaptation of the proof in Theorem 3.3 of [13] shows that the fact that the isomorphism $\theta$ is induced by a continuous orbit equivalence $h$ forces the groups $G_{U}$ and $G_{V}^{\prime}$ to have equal indices in $G$ and $G^{\prime}$ respectively. In Definition 6.8 of return equivalence, the homeomorphism $h_{U}$ need not be induced by a homeomorphism of the space $\mathfrak{X}$, so there is no requirement that the groups $H_{U}$ and $H_{V}^{\prime}$ have the same index with respect to any larger groups.

6.3. Rigidity for Cantor actions. The approach in the literature to proving rigidity for Cantor actions is to assume that both actions are free on an invariant dense subset $\mathfrak{Z} \subset \mathfrak{X}$, then observe that the function $\alpha$ in Definition 6.1 satisfies a cocycle identity on $\mathfrak{Z}$, hence on all of $\mathfrak{X}$ by continuity. The map $\alpha: G \times \mathfrak{X} \rightarrow H$ is called the "orbit cocycle" for the orbit equivalence.

Then either a cohomological assumption as in $\mathrm{Li}$ [38, or a dynamical assumption as in Cortez and Medynets [13, is used to show that the cocycle $\alpha$ is cohomologous to a constant cocycle, which implies that the conditions of Definition 6.3 are satisfied.

The results of Boyle and Tamiyama in [7] can be interpreted as saying that a minimal Cantor action by $\mathbb{Z}$ is rigid, as stated in [38, Theorem 3.2]. As $G$ is abelian and the action is faithful in this case, there is a well-defined orbit cocycle, and the authors show that it is cohomologous to a constant.

Cortez and Medynets showed that free equicontinuous Cantor actions are rigid in [13, Theorem 3.3], and in fact their proof directly extends to show:

THEOREM 6.9. Let $G, G^{\prime}$ be finitely generated groups, and $(\mathfrak{X}, G, \Phi)$ and $\left(\mathfrak{X}^{\prime}, G^{\prime}, \Psi\right)$ be faithful Cantor actions. Suppose that the actions $\Phi$ and $\Psi$ are topologically free. Then the actions are continuous orbit equivalent if and only they are virtually rigid in the sense of Definition 6.7.

The proof of this result in [13] does not explicitly discuss the orbit cocycle. Rather, it uses the odometer model for an equicontinuous action to construct the isomorphism $\theta_{U}$ in Definition 6.7 directly from the action.

For $G$ a finitely generated group, Theorems 1.3 and 1.4 in $\mathrm{Li} 38$ give conditions on a topologically free Cantor action which imply that the orbit cocycle for the action is cohomologous to a constant cocycle. The discussion in [38, Section 4] explains the passage from a constant cocycle to a conjugacy. 
The methods in [13, 38] were used in the authors' work [35, Section 4] to show that stable actions which are continuous orbit equivalent are return equivalent. We also use these techniques in the proof of the following result, which will be used in the proof of our rigidity result Theorem 6.18

THEOREM 6.10. Let $h: \mathfrak{X} \rightarrow \mathfrak{X}^{\prime}$ be a continuous orbit equivalence between Cantor actions $(\mathfrak{X}, G, \Phi)$ and $\left(\mathfrak{X}^{\prime}, G^{\prime}, \Psi\right)$. If $G$ is finitely generated and $\left(\mathfrak{X}^{\prime}, G^{\prime}, \Psi\right)$ is stable, then $(\mathfrak{X}, G, \Phi)$ is stable.

Proof. Following the discussion in Section 6.1, we can assume that $\mathfrak{X}=\mathfrak{X}^{\prime}$ and $h$ is the identity map. Fix a basepoint $x \in \mathfrak{X}$. Then by Theorem 5.3. there exists $V_{1} \subset \mathfrak{X}$ adapted to the action $\Psi$ with $x \in V_{1}$, such that the action of $H_{1}^{\prime}=\Psi\left(G_{V_{1}}^{\prime}\right) \subset \operatorname{Homeo}\left(V_{1}\right)$ on $V_{1}$ is topologically free. Let $\mathcal{Z} \subset V_{1}$ be a dense subset which is $H_{1}^{\prime}$-invariant and the restriction of the action of $H_{1}^{\prime}$ to $\mathcal{Z}$ is free.

Let $W \subset \mathfrak{X}$ be adapted set for the action $\Phi$ with $x \in W \subset V_{1}$. Let $G_{W} \subset G$ be defined as in (3).

Let $\alpha: G \times \mathfrak{X} \rightarrow G^{\prime}$ be the orbit function which satisfies the relation (37), and let $\alpha_{W}: G_{W} \times W \rightarrow G^{\prime}$ denote its restriction to $W$.

For each $g \in G_{W}$ and $y \in W$ we have $\Phi(g)(y) \in W$. Let $h \in G^{\prime}$ be such that $\Psi(h)(y)=\Phi(g)(y)$, then $W \subset V_{1}$ implies that $\Psi(h)\left(V_{1}\right) \cap V_{1} \neq \emptyset$ hence $\Psi(h) \in H_{1}^{\prime}=\Psi_{V}\left(G_{V_{1}}^{\prime}\right) \subset \operatorname{Homeo}\left(V_{1}\right)$. That is, the restriction of $\alpha$ to $G_{W} \times W$ induces a map $\widehat{\alpha}_{W}=\Psi_{V^{\prime}} \circ \alpha: G_{W} \times W \rightarrow H_{1}^{\prime}$, where $\Psi_{V^{\prime}}: G_{1}^{\prime} \rightarrow H_{1}^{\prime}$.

The action of $H_{1}^{\prime}$ on $V_{1}$ is topologically free, so the proof of [38, Lemma 2.8] adapts to yield:

LEMMA 6.11. $\widehat{\alpha}_{W}: G_{W} \times W \rightarrow H_{1}^{\prime}$ satisfies the cocycle identity (39) for the restricted action $\Phi_{W}: G_{W} \times W \rightarrow W$.

We next show that a properly chosen restriction of the cocycle $\widehat{\alpha}_{W}$ is a coboundary. The proof of this fact follows closely the proof of [13, Theorem 3.3], with the variation that we only assume the action of $H_{1}^{\prime}$ is topologically free, and do not assume the action of $G_{W}$ on $W$ is topologically free.

Since $G$ is finitely generated, the same holds for the subgroup $G_{W}$ of finite index. Choose a symmetric generating set $\left\{g_{i} \mid 1 \leq i \leq m\right\}$ for $G_{W}$. That is, each $g \in G_{W}$ can be written as a product $g=g_{i_{1}} g_{i_{2}} \cdots g_{i_{\nu}}$ where each $1 \leq i_{\ell} \leq m$. The map $\alpha: G \times \mathfrak{X} \rightarrow G^{\prime}$ is continuous, and $G^{\prime}$ is discrete, hence $\alpha_{W}$ is locally constant. Thus, there exists $\delta_{1}>0$ so that

$$
\alpha_{W}\left(g_{i}, y\right)=\alpha_{W}\left(g_{i}, z\right) \text { for } 1 \leq i \leq m \text { and } y, z \in \mathfrak{X} \text { with } d_{\mathfrak{X}}(y, z)<\delta_{1} .
$$

Let $W^{\prime} \subset W$ be an adapted set such that $x \in W^{\prime}$ and $\operatorname{diam}\left(g \cdot W^{\prime}\right)<\delta_{1}$ for all $g \in G_{W}$.

The collection $\mathcal{W}^{\prime} \equiv\left\{g \cdot W^{\prime} \mid g \in G_{W}\right\}$ is a finite clopen partition of $W$, so there exists $0<\delta_{2}<\delta_{1}$ such that for any $y \in W$ there exists $g \in G_{W}$ such that $B_{d_{\mathfrak{X}}}\left(y, \delta_{2}\right) \subset g \cdot W^{\prime}$, and $g \cdot W^{\prime}$ is the unique element of the partition $\mathcal{W}^{\prime}$ which contains it.

By the uniform continuity of the action $\Phi$, there exists $0<\delta_{3} \leq \delta_{2}$ such that

$$
d_{\mathfrak{X}}(\Phi(g)(y), \Phi(g)(z))<\delta_{2} \text { for all } y, z \in \mathfrak{X} \text { with } d_{\mathfrak{X}}(y, z)<\delta_{3} \text {, for all } g \in G \text {. }
$$

Choose an adapted set $U_{1}$ such that $x \in U_{1} \subset W^{\prime}$ and $\operatorname{diam}\left(g^{\prime} \cdot U_{1}\right)<\delta_{3}$ for all $g^{\prime} \in G_{U_{1}}$. That is, once $W^{\prime}$ has been chosen, choose $U_{1}$ sufficiently small so that all orbits of points in $U_{1}$ under the action of $\Phi\left(G_{W}\right)$ have well-defined codings with respect to the partition $\mathcal{W}^{\prime}$ of $W$ formed by the translates of $W^{\prime}$. (The coding method is discussed in detail in [9, Section 6].)

Set $G_{1}=G_{U_{1}} \subset G_{W}$, let $H_{1}=\Phi_{U_{1}}\left(G_{1}\right) \subset \operatorname{Homeo}\left(U_{1}\right)$, and let $\Phi_{1}: G_{1} \rightarrow H_{1}$ denote the quotient map. We also denote the restricted action of $G_{1}$ by $\Phi_{1}: G_{1} \times U_{1} \rightarrow U_{1}$. Let $\widehat{\alpha}_{1}: G_{1} \times U_{1} \rightarrow H_{1}^{\prime}$ denote the cocycle over $\Phi_{1}$ obtained by restricting $\widehat{\alpha}_{W}$. The next result shows that $\widehat{\alpha}_{1}$ is a coboundary. The proof below uses a key idea from the proof of [13, Theorem 3.3].

PROPOSITION 6.12. $\widehat{\alpha}_{1}: G_{1} \times U_{1} \rightarrow H_{1}^{\prime}$ is induced by a homomorphism $\widehat{\theta}_{1}: G_{1} \rightarrow H_{1}^{\prime}$. That is, for $g \in G_{1}$ we have $\Psi\left(\widehat{\alpha}_{1}(g, x)\right)=\widehat{\theta}_{1}(g)$, and thus

$$
\Phi(g)(y)=\widehat{\theta}_{1}(g)(y) \quad \text { for all } y \in U_{1} .
$$


Proof. We first show that for $g \in G_{1}$ the function $\widehat{\alpha}_{1}(g, y)$ is constant in $y \in U_{1}$.

Recall that $\left\{g_{i} \mid 1 \leq i \leq m\right\}$ is a symmetric generating set for $G_{W}$. By the choice of $\delta_{1}$, for $d_{\mathfrak{X}}(y, z)<\delta_{1}$ and $1 \leq i \leq m$, we have $\widehat{\alpha}_{1}\left(g_{i}, y\right)=\widehat{\alpha}_{1}\left(g_{i}, z\right)$. Thus by the choice of $W^{\prime}$ and (42), for any $g^{\prime} \in G_{W}$, the value of $\widehat{\alpha}_{W}\left(g_{i}, y\right)$ is constant for $y \in g^{\prime} \cdot W^{\prime}$.

Then by the choice of $\delta_{3}$ so that (43) holds, and the choice of $U_{1}$ so that $\operatorname{diam}\left(g^{\prime} \cdot U_{1}\right)<\delta_{3}$ for $g^{\prime} \in G_{W}$, then for $y, z \in U_{1}$ we have $g^{\prime} \cdot y \in g^{\prime} \cdot U_{1}$ and $g^{\prime} \cdot z \in g^{\prime} \cdot U_{1}$. Thus, $g^{\prime} \cdot y$ and $g^{\prime} \cdot z$ are contained in the same set of the partition $\mathcal{W}^{\prime}$, hence $\widehat{\alpha}_{1}\left(g_{i}, g^{\prime} \cdot y\right)=\widehat{\alpha}_{1}\left(g_{i}, g^{\prime} \cdot z\right)$ for any $1 \leq i \leq m$.

We now apply this recursively to the elements of $G_{1}$. Let $g \in G_{1}$, then $g=g_{i_{1}} \cdots g_{i_{\nu}} \in G_{1}$. Set $g^{\prime}=g_{i_{2}} \cdots g_{i_{\nu}}$ then for $y, z \in \mathcal{Z} \cap U_{1}$,

$$
\begin{aligned}
& \widehat{\alpha}_{1}(g, y)=\widehat{\alpha}_{1}\left(g_{i_{1}} g^{\prime}, y\right)=\widehat{\alpha}_{W}\left(g_{i_{1}}, g^{\prime} \cdot y\right) \circ \widehat{\alpha}_{W}\left(g^{\prime}, y\right) \\
& \quad=\widehat{\alpha}_{W}\left(g_{i_{1}}, g^{\prime} \cdot z\right) \circ \widehat{\alpha}_{W}\left(g^{\prime}, z\right)=\widehat{\alpha}_{W}\left(g_{i_{1}} g^{\prime}, z\right)=\widehat{\alpha}_{1}(g, z) .
\end{aligned}
$$

As the values of $\widehat{\alpha}_{W}(g, y) \in H_{1}^{\prime}$ and $\widehat{\alpha}_{W}(g, z) \in H_{1}^{\prime}$ are defined using the identity (37), the identity (45) holds for all $y, z \in U_{1}$ as the closure $\overline{\mathcal{Z} \cap U_{1}}=U_{1}$.

Thus, the calculation (45) shows that the value of $\widehat{\alpha}_{1}(g, y) \in H_{1}^{\prime}$ does not depend on the choice of $y \in U_{1}$. We are given that $\Phi(g)(y) \in U_{1}$ for all $g \in G_{1}$, as $U_{1}$ is adapted to the action $\Phi$. Thus, for $g, g^{\prime} \in G_{1}$ and $y \in U_{1}$, we have $z=g^{\prime} \cdot y \in U_{1}$ and so

$$
\widehat{\alpha}_{1}\left(g g^{\prime}, y\right)=\widehat{\alpha}_{1}(g, z) \widehat{\alpha}_{1}\left(g^{\prime}, y\right)=\widehat{\alpha}_{1}(g, y) \widehat{\alpha}_{1}\left(g^{\prime}, y\right) .
$$

Fix $y \in U_{1}$ and define $\widehat{\theta}_{1}: G_{1} \rightarrow H_{1}^{\prime}$ by setting $\widehat{\theta}_{1}(g)=\widehat{\alpha}_{1}(g, y)$, then (46) says that $\widehat{\theta}_{1}$ is a group homomorphism. Then (44) is just a restatement of the identity (37).

Recall that $H_{1}=\Phi_{1}\left(G_{1}\right) \subset \operatorname{Homeo}\left(U_{1}\right)$, and $H_{1}^{\prime}=\Psi\left(G_{V_{1}}^{\prime}\right) \subset \operatorname{Homeo}\left(V_{1}\right)$.

COROLLARY 6.13. $\widehat{\theta}_{1}$ induces a homomorphism $\theta_{1}: H_{1} \rightarrow H_{1}^{\prime}$.

Proof. Suppose that $g, g^{\prime} \in G_{1}$ satisfy $\Phi(g)\left|U_{1}=\Phi\left(g^{\prime}\right)\right| U_{1} \in H_{1} \subset$ Homeo $\left(U_{1}\right)$. Then by the defining identity (37), for $y \in \mathcal{Z} \cap U_{1}$ the action of $H_{1}^{\prime}$ is free on the orbit of $y$, hence $\widehat{\theta}_{1}(g)=\widehat{\alpha}_{1}(g, y)=$ $\widehat{\alpha}_{1}\left(g^{\prime}, y\right)=\widehat{\theta}_{1}\left(g^{\prime}\right) \in H_{1}^{\prime}$. Thus, $\widehat{\theta}_{1}$ induces a homomorphism $\theta_{1}: H_{1} \rightarrow H_{1}^{\prime}$.

6.4. The induced map. Recall that we are assuming the hypotheses of Theorem [6.10, so that $h: \mathfrak{X} \rightarrow \mathfrak{X}^{\prime}$ is a continuous orbit equivalence between Cantor actions $(\mathfrak{X}, G, \Phi)$ and $\left(\mathfrak{X}^{\prime}, G^{\prime}, \Psi\right)$, that $G$ is finitely generated, and $\left(\mathfrak{X}^{\prime}, G^{\prime}, \Psi\right)$ is stable.

The conclusion of Corollary 6.13 states that the identity (37), which defines the orbit equivalence between actions $(\mathfrak{X}, G, \Phi)$ and $\left(\mathfrak{X}, G^{\prime}, \Psi\right)$, induces a homomorphism $\theta_{1}: H_{1} \rightarrow H_{1}^{\prime}$ but does not show that $\theta_{1}$ is an isomorphism. We next show that the image of $\theta_{1}$ is "large enough" in the profinite topology defined by adapted bases for the actions, so that $\theta_{1}$ induces a map between wellchosen odometer models for the actions $\Phi$ and $\Psi$. The idea is to recursively construct adapted neighborhood bases for these actions so that we can recursively apply the techniques of the proof of Proposition 6.12. First, set $U_{0}=V_{0}=\mathfrak{X}$, and let $U_{1} \subset V_{1}$ be as in the proofs of Lemma 6.11 and Proposition 6.12. Then recursively choose:

(1) $V_{\ell+1}$ adapted to $\Psi$, with $x \in V_{\ell+1} \subset U_{\ell}$ for $\ell \geq 1$;

(2) $U_{\ell}$ adapted to $\Phi$, with $x \in U_{\ell} \subset V_{\ell}$ for $\ell \geq 2$;

such that $\cap_{\ell \geq 1} U_{\ell}=\cap_{\ell \geq 1} V_{\ell}=\{x\}$. We obtain adapted neighborhood bases $\mathcal{U}=\left\{U_{\ell} \subset \mathfrak{X} \mid \ell \geq 0\right\}$ for $(\mathfrak{X}, G, \Phi)$ at $x$, and $\mathcal{V}=\left\{V_{\ell} \subset \mathfrak{X} \mid \ell \geq 0\right\}$ for $\left(\mathfrak{X}, G^{\prime}, \Psi\right)$ at $x$.

Let $G_{\ell} \equiv G_{U_{\ell}} \subset G$ for $\ell \geq 0$, then $\mathcal{G}_{\mathcal{U}}=\left\{G_{\ell} \mid \ell \geq 0\right\}$ is a group chain associated to the action $\Phi$.

Let $G_{\ell}^{\prime} \equiv G_{V_{\ell}}^{\prime} \subset G^{\prime}$ for $\ell \geq 0$, then $\mathcal{G}_{\mathcal{V}}^{\prime}=\left\{G_{\ell}^{\prime} \mid \ell \geq 0\right\}$ is a group chain associated to the action $\Psi$.

Set $H_{\ell} \equiv H_{U_{\ell}}=\Phi\left(G_{\ell}\right) \mid U_{\ell} \subset \operatorname{Homeo}\left(U_{\ell}\right)$, and $H_{\ell}^{\prime} \equiv H_{V_{\ell}}^{\prime}=\Psi\left(G_{\ell}^{\prime}\right) \mid V_{\ell} \subset \operatorname{Homeo}\left(V_{\ell}\right)$. 
Let $\left(U_{1}, G_{1}, \Phi_{1}\right)$ denote the restricted action $\Phi_{1}: G_{1} \times U_{1} \rightarrow U_{1}$ of $\Phi$. Let $\bar{\Phi}_{1}: H_{1} \times U_{1} \rightarrow U_{1}$ be the induced action by $H_{1} \equiv \Phi_{1}\left(G_{1}\right) \subset \operatorname{Homeo}\left(U_{1}\right)$.

Let $\left(V_{1}, G_{1}^{\prime}, \Psi_{1}\right)$ denote the restricted action $\Psi_{1}: G_{1}^{\prime} \times V_{1} \rightarrow V_{1}$ of $\Psi$. Let $\bar{\Psi}_{1}: H_{1}^{\prime} \times V_{1} \rightarrow V_{1}$ be the induced action by $H_{1}^{\prime} \equiv \Psi_{1}\left(G_{1}^{\prime}\right) \subset \operatorname{Homeo}\left(V_{1}\right)$.

We next use the existence of the map $\beta$ defined by the identity (38) to prove the following result, which is used to show that the map $\theta_{1}$ induces a map between the odometer models for the induced Cantor actions $\left(U_{1}, H_{1}, \bar{\Phi}_{1}\right)$ and $\left(V_{1}, H_{1}^{\prime}, \bar{\Psi}_{1}\right)$. Note that it is not assumed that the action of $H_{1}$ on $U_{1}$ is topologically free, and as a consequence the choice of the map $\beta$ in (38) need not be unique. Correspondingly, the map $\theta_{1}$ need not be a monomorphism.

LEMMA 6.14. For all $\ell \geq 1$, the subgroup $H_{\ell}^{\prime} \subset H_{1}^{\prime}$ defined as follows, satisfies

$$
H_{\ell+1}^{\prime} \equiv \Psi_{1}\left(G_{\ell+1}^{\prime}\right) \subset \widehat{\theta}_{1}\left(G_{\ell}\right)=\theta_{1} \circ \Phi_{1}\left(G_{\ell}\right) \subset \Psi_{1}\left(G_{\ell}^{\prime}\right) \equiv H_{\ell}^{\prime} \subset H_{1}^{\prime} .
$$

Proof. Fix $\ell \geq 1$, let $k \in G_{\ell+1}^{\prime}$ and $y \in V_{\ell+1}$, then by the identity (38) there exists $g=\beta(k, y) \in G$ and an open set $V_{k, y} \subset \mathfrak{X}$, such that $\Phi(g)(z)=\Psi(k)(z)$ for all $z \in V_{k, y}$. Note that we are not assuming that the function $\beta$ is a cocycle, just the fact that it exists. Then by the definition of $\widehat{\alpha}_{1}: G_{1} \times U_{1} \rightarrow H_{1}^{\prime}$ as the restriction of $\widehat{\alpha}_{W}$, we have that $\widehat{\alpha}_{1}(g)=\Psi(k)$ when restricted to $V_{k, y}$.

Recall that the action of $H_{1}^{\prime}=\Psi_{1}\left(G_{V_{1}}^{\prime}\right)$ on $V_{1}$ is topologically free, and $H_{1}^{\prime}$ acts freely on the dense $H_{1}^{\prime}$-invariant subset $\mathcal{Z} \subset V_{1}$. The subset $V_{k, y} \cap V_{1}$ is open in $V_{1}$ and contained in the closure of $\mathcal{Z} \cap V_{k, y} \cap V_{1}$, hence the maps $\Phi(g)$ and $\Psi(k)$ agree on an open subset of $V_{1}$, hence agree on $V_{1}$ since the homomorphism $\theta_{1}$ does not depend on the choice of $U_{1}$.

It is given that $k \in G_{\ell+1}^{\prime}$ and $y \in V_{\ell+1} \subset U_{\ell}$, so $\Psi(k)(y) \in V_{\ell+1} \subset U_{\ell}$, and thus $\Phi(g)\left(U_{\ell}\right) \cap U_{\ell} \neq \emptyset$, hence $g \in G_{\ell}$. Thus, by (44) we have

$$
\Psi(k)\left|V_{1}=\Phi(g)\right| V_{1}=\widehat{\theta}_{1}(g) \mid V_{1} \in \widehat{\theta}_{1}\left(G_{\ell}\right),
$$

which establishes the first inclusion in (47).

To show the second inclusion in (47), let $g \in G_{\ell}$ then note that $\Phi(g)\left(U_{\ell}\right) \cap U_{\ell} \neq \emptyset$. Then by (44) we have $\widehat{\theta}_{1}(g) \cdot U_{\ell} \cap U_{\ell} \neq \emptyset$, hence $\widehat{\theta}_{1}(g) \cdot V_{\ell} \cap V_{\ell} \neq \emptyset$ and so $\widehat{\theta}_{1}(g) \in H_{\ell}^{\prime}$.

Next, we examine the relation between the discriminant groups for the Cantor actions $(\mathfrak{X}, \mathfrak{G}(\Phi), \widehat{\Phi})$ and $\left(U_{k}, H_{k}, \bar{\Phi}_{k}\right)$, for $k \geq 1$, where $\mathcal{U}=\left\{U_{\ell} \subset \mathfrak{X} \mid \ell \geq 0\right\}$ is the adapted neighborhood basis at $x$ chosen above for $(\mathfrak{X}, G, \Phi)$. Let $\widehat{G}_{\infty}$ be the profinite group associated to the group chain $\mathcal{G}_{\mathcal{U}}$, and let $\widehat{\Phi}_{x}: \widehat{G}_{\infty} \times X_{\infty} \rightarrow X_{\infty}$ be the associated action. We show that the wild property for this action is preserved by restriction.

By Theorem 3.2, there is a topological isomorphism $\widehat{\Theta}: \mathfrak{G}(\Phi) \rightarrow \widehat{G}_{\infty}$, which conjugates the action $\widehat{\Phi}$ to the action $\widehat{\Phi}_{x}$ so we may make all calculations in terms of the action $\widehat{\Phi}_{x}: \widehat{G}_{\infty} \times X_{\infty} \rightarrow X_{\infty}$.

Recall that for $\ell \geq 1$, the clopen set $\widehat{U}_{\ell} \equiv \widehat{G}_{\ell} / \mathcal{D}_{x} \subset \widehat{G}_{\infty} / \mathcal{D}_{x} \cong X_{\infty}$ is adapted to the action $\widehat{\Phi}_{x}$, with stabilizer $\widehat{G}_{\ell} \subset \widehat{G}_{\infty}$ defined by (17). Recall that $\Phi_{\ell}: G_{\ell} \times \widehat{U}_{\ell} \rightarrow \widehat{U}_{\ell}$ denotes the restriction of the action $\Phi_{x}$ to $\widehat{U}_{\ell}$. Now for $\ell^{\prime} \geq \ell \geq 0$, define

$$
H_{\ell, \ell^{\prime}} \equiv \Phi_{\ell}\left(G_{\ell^{\prime}}\right) \subset \Phi_{\ell}\left(G_{\ell}\right) \subset \operatorname{Homeo}\left(\widehat{U}_{\ell}\right),
$$

and let $\widehat{H}_{\ell, \ell^{\prime}}$ denote the closure of $H_{\ell, \ell^{\prime}}$ in the uniform topology on $\operatorname{Homeo}\left(\widehat{U}_{\ell}\right)$. Note that $H_{\ell, \ell}=H_{\ell}$ and $\widehat{H}_{\ell, \ell}=\widehat{H}_{\ell}$ as defined previously. Then the restricted action $\Phi_{\ell}$ induces a homomorphism $\widehat{\Phi}_{\ell}: \widehat{G}_{\ell} \rightarrow \widehat{H}_{\ell}$ which is a surjection, as seen using the same method of proof as for Lemma 2.5

Recall that for all $\ell \geq 0, \mathcal{D}_{x} \subset \widehat{G}_{\ell}$ so there is a well defined isotropy action $\rho_{\ell}: \mathcal{D}_{x} \rightarrow \operatorname{Homeo}\left(\widehat{U}_{\ell}\right)$. Then by (22) we have $K_{\ell}=\operatorname{ker}\left\{\rho_{\ell}: \mathcal{D}_{x} \rightarrow \operatorname{Homeo}\left(\widehat{U}_{\ell}\right)\right\}$. Thus, $K_{\ell}$ is a normal subgroup of $\mathcal{D}_{x}$ and in particular, $K_{\ell}$ is normal in $K_{\ell^{\prime}}$ for $\ell^{\prime}>\ell$.

For $k \geq 0$, by Lemma 3.11 we have $\mathcal{D}_{x}=\bigcap_{\ell \geq 0} \widehat{G}_{\ell}=\bigcap_{\ell \geq k} \widehat{G}_{\ell}$. 
For $k \geq 0$, recall that $H_{k}=\Phi_{k}\left(G_{k}\right) \subset \operatorname{Homeo}\left(\widehat{U}_{k}\right)$, and let $\bar{\Phi}_{k}: H_{k} \times \widehat{U}_{k} \rightarrow \widehat{U}_{k}$ denote the quotient action induced from the restricted action $\left(\widehat{U}_{k}, G_{k}, \Phi_{k}\right)$. Then we have:

PROPOSITION 6.15. For $k \geq 0$, the stabilizer direct limit group $\Upsilon_{s}\left(\bar{\Phi}_{k}\right)$ for the induced action $\left(\widehat{U}_{k}, H_{k}, \bar{\Phi}_{k}\right)$ is represented by the quotient group chain $\left\{K_{\ell} / K_{k} \mid \ell \geq k\right\}$.

Proof. First, observe that $\mathcal{H}_{k} \equiv\left\{H_{k, \ell} \mid \ell \geq k\right\}$ is an adapted group chain for the action $\left(\widehat{U}_{k}, H_{k}, \bar{\Phi}_{k}\right)$ at $x \in \widehat{U}_{k}$. Recall that $\widehat{H}_{k} \subset \operatorname{Homeo}\left(\widehat{U}_{k}\right)$ is the closure of the group $H_{k}$.

Let $A_{k}=\operatorname{ker}\left\{\widehat{\Phi}_{k}: \widehat{G}_{k} \rightarrow \widehat{H}_{k}\right\}$, which is a closed normal subgroup of $\widehat{G}_{k}$. Then for $\ell>k$ we have $\widehat{H}_{k, \ell} \cong \widehat{G}_{\ell} /\left(\widehat{G}_{\ell} \cap A_{k}\right)$, and the discriminant $\overline{\mathcal{D}}_{k}$ of the action $\left(\widehat{U}_{k}, H_{k}, \bar{\Phi}_{k}\right)$ is given by $\overline{\mathcal{D}}_{k}=\bigcap_{\ell \geq k} \widehat{H}_{k, \ell}$.

Suppose $\widehat{h} \in \mathcal{D}_{x}$ then the image $\bar{\Phi}_{k}(\widehat{h}) \in \widehat{H}_{k, \ell}$ for all $\ell \geq k$, hence $\widehat{\Phi}_{k}(\widehat{h}) \in \overline{\mathcal{D}}_{k}$. Moreover, as $\widehat{G}_{k}$ is sequentially compact, the restricted map $\widehat{\Phi}_{k}: \mathcal{D}_{x} \rightarrow \overline{\mathcal{D}}_{k}$ is onto, with kernel the closed normal subgroup $K_{k} \equiv \mathcal{D}_{x} \cap A_{k}$. Thus, there is an isomorphism $\widehat{\Phi}_{k}: \mathcal{D}_{x} / K_{k} \cong \overline{\mathcal{D}}_{k}$.

For each $\ell>k$, let $\bar{\rho}_{k, \ell}: \overline{\mathcal{D}}_{k} \rightarrow \widehat{H}_{\ell}$ be the restriction of the map $\bar{\Phi}_{k}: \widehat{H}_{k, \ell} \rightarrow \widehat{H}_{\ell} \subset \operatorname{Homeo}\left(\widehat{U}_{\ell}\right)$, and let $\bar{K}_{k, \ell}=\operatorname{ker}\left\{\bar{\Phi}_{k}\right\}$. Then the stabilizer group $\Upsilon_{s}\left(\bar{\Phi}_{k}\right)$ is represented by the chain $\left\{\bar{K}_{k, \ell} \mid \ell \geq k\right\}$.

Let $\widehat{k} \in \bar{K}_{k, \ell}$ and so $\widehat{k}$ acts as the identity on $\widehat{U}_{\ell}$. Choose $\widehat{h} \in \mathcal{D}_{x}$ with $\widehat{\Phi}_{k}(\widehat{h})=\widehat{k}$, then $\widehat{h}$ also acts as the identity on $\widehat{U}_{\ell}$, hence $\widehat{h} \in K_{\ell}$. Moreover, if $\widehat{h} \in K_{\ell}$ and $\widehat{\Phi}_{k}(\widehat{h})=i d$ then $\widehat{h} \in K_{\ell} \cap A_{k}=K_{k}$. It follows that $\bar{K}_{k, \ell} \cong K_{\ell} / K_{k}$ for $\ell \geq k$, which yields the claim.

COROLLARY 6.16. For $k \geq 1,(\mathfrak{X}, G, \Phi)$ is wild if and only if $\left(\widehat{U}_{k}, H_{k}, \bar{\Phi}_{k}\right)$ is wild.

Proof. Let $\ell \geq 0$. Then $(\mathfrak{X}, G, \Phi)$ is a wild action, if and only if the chain of subgroups $\left\{K_{\ell} \mid \ell \geq 0\right\}$ as above is unbounded, if and only if the chain of quotient groups $\left\{K_{\ell} / K_{k} \mid \ell \geq k\right\}$ is unbounded. Then by the proof of Proposition 6.15 above, we have $\bar{K}_{k, \ell} \cong K_{\ell} / K_{k}$, so the action is wild if and only if the chain $\left\{\bar{K}_{k, \ell} \mid \ell \geq k\right\}$ is unbounded, if and only if the stabilizer group $\Upsilon_{s}\left(\bar{\Phi}_{k}\right)$ is unbounded.

We can now complete the proof of Theorem 6.10, We are given the Cantor actions $(\mathfrak{X}, G, \Phi)$ and $\left(\mathfrak{X}, G^{\prime}, \Psi\right)$, where $G$ and $G^{\prime}$ are finitely generated groups, $\Phi$ and $\Psi$ have the same orbits, and satisfy the conditions (37) and (38). We assume that the action $(\mathfrak{X}, G, \Phi)$ is wild, and derive a contradiction to the assumption that the action $\left(\mathfrak{X}^{\prime}, G^{\prime}, \Psi\right)$ is stable.

By Proposition 6.12, there is a clopen set $U_{1} \subset \mathfrak{X}$ adapted to the action $\Phi$, a clopen set $V_{1} \subset \mathfrak{X}$ adapted to the action $\Psi$, and a homomorphism $\theta_{1}: H_{1} \rightarrow H_{1}^{\prime}$ which satisfies the condition (44).

Let $\mathcal{U}=\left\{U_{\ell} \subset \mathfrak{X} \mid \ell \geq 0\right\}$ be an adapted neighborhood basis for $(\mathfrak{X}, G, \Phi)$ at $x$, and let $\mathcal{V}=\left\{V_{\ell} \subset\right.$ $\mathfrak{X} \mid \ell \geq 0\}$ be an adapted neighborhood basis for $\left(\mathfrak{X}, G^{\prime}, \Psi\right)$ at $x$, where $U_{1}$ and $V_{1}$ are chosen so that Proposition 6.12 holds, and the chains $\mathcal{U}$ and $\mathcal{V}$ chosen as above so that the conclusions of Lemma 6.14 hold. We use the notation of the proofs of these results in the following as well.

Let $\mathcal{G}_{\mathcal{U}}=\left\{G_{\ell} \mid \ell \geq 0\right\}$ be the group chain associated to $\mathcal{U}$, and let $\mathcal{H}_{\mathcal{U}}=\left\{H_{1, \ell} \mid \ell \geq 1\right\}$ be the group chain for the induced action $\left(U_{1}, H_{1}, \bar{\Phi}_{1}\right)$.

Let $\widehat{G}_{\infty}$ be the profinite group associated to the group chain $\mathcal{G}_{\mathcal{U}}$, with discriminant group $\mathcal{D}_{x} \subset \widehat{G}_{\infty}$.

Let $\widehat{H}_{\infty}$ be the profinite group associated to the group chain $\mathcal{H}_{\mathcal{U}}$, with discriminant group $\overline{\mathcal{D}}_{1} \subset \widehat{H}_{\infty}$.

Let $\mathcal{K}=\left\{K_{\ell} \mid \ell \geq 0\right\}$ be the group chain representing $\Upsilon_{s}^{x}(\Phi)$, and let $\overline{\mathcal{K}} \cong\left\{\bar{K}_{1, \ell} \cong K_{\ell} / K_{1} \mid \ell \geq 1\right\}$ be the group chain representing $\Upsilon_{s}^{x}\left(\bar{\Phi}_{1}\right)$. Then by Corollary 6.16, the chain $\overline{\mathcal{K}}$ is unbounded.

Next, let $\mathcal{G}_{\mathcal{V}}^{\prime}=\left\{G_{\ell}^{\prime} \mid \ell \geq 0\right\}$ be the group chain associated to $\mathcal{V}$, and let $\mathcal{H}_{\mathcal{V}}^{\prime}=\left\{H_{1, \ell}^{\prime} \mid \ell \geq 1\right\}$ be the group chain for the induced action $\left(V_{1}, H_{1}^{\prime}, \bar{\Psi}_{1}\right)$. Note that $H_{1,1}^{\prime}=H_{1}^{\prime}$.

Let $\widehat{G}_{\infty}^{\prime}$ be the profinite group associated to the group chain $\mathcal{G}_{\mathcal{V}}^{\prime}$, with discriminant group $\mathcal{D}_{x}^{\prime} \subset \widehat{G}_{\infty}^{\prime}$. Let $\widehat{H}_{\infty}^{\prime}$ be the profinite group associated to the group chain $\mathcal{H}_{\mathcal{V}}^{\prime}$, with discriminant group $\overline{\mathcal{D}}_{1}^{\prime} \subset \widehat{H}_{\infty}^{\prime}$. 
Let $\mathcal{K}^{\prime}=\left\{K_{\ell}^{\prime} \mid \ell \geq 0\right\}$ be the group chain representing $\Upsilon_{s}^{x}(\Psi)$, and let $\overline{\mathcal{K}}^{\prime}=\left\{\bar{K}_{1, \ell}^{\prime} \cong K_{\ell}^{\prime} / K_{1}^{\prime} \mid \ell \geq 1\right\}$ be the group chain representing $\Upsilon_{s}^{x}\left(\bar{\Psi}_{1}\right)$.

The homomorphism $\theta_{1}: H_{1} \rightarrow H_{1}^{\prime}$ satisfies the condition (47), which implies that

$$
H_{1, \ell+1}^{\prime} \subset \theta_{1}\left(H_{1, \ell}\right) \subset H_{1, \ell}^{\prime}
$$

so induces a map $\widehat{\tau}_{1}: \widehat{H}_{\mathcal{U}} \rightarrow \widehat{H}_{\mathcal{V}}^{\prime}$. Then (50) and Lemma 3.11 imply that $\widehat{\tau}_{1}$ restricts to a surjection $\widehat{\tau}_{1}: \overline{\mathcal{D}}_{1} \rightarrow \overline{\mathcal{D}}_{1}^{\prime}$ which satisfies $\widehat{\tau}_{1}\left(\bar{K}_{\ell}\right)=\bar{K}_{\ell}^{\prime}$. As a result, by Theorem 3.7 we obtain maps

$$
\mathfrak{X} \supset U_{1} \cong \widehat{H}_{\infty} / \overline{\mathcal{D}}_{1} \stackrel{\widehat{\tau}_{*}}{\longrightarrow} \widehat{H}_{\infty}^{\prime} / \overline{\mathcal{D}}_{1}^{\prime} \cong V_{1} \subset \mathfrak{X} .
$$

That is, $\widehat{\tau}_{*}: U_{1} \rightarrow V_{1}$ is the map induced from the homomorphism $\widehat{\tau}_{1}$ which is $H_{1}$-equivariant.

We claim that if $\widehat{k} \in \bar{K}_{\ell+1}$ and $\widehat{k} \notin \bar{K}_{\ell}$, then $\widehat{\tau}_{1}(\widehat{k}) \notin \bar{K}_{\ell-1}^{\prime}$, hence $\bar{K}_{\ell-1}^{\prime} \neq \bar{K}_{\ell+1}^{\prime}$. Let $\widehat{h} \in \mathcal{D}_{x}$ be chosen so that it has image $\widehat{k} \in \overline{\mathcal{D}}_{1}$. Then $\widehat{k} \notin \bar{K}_{\ell}$ implies that the restricted action of $\Phi_{\ell}(\widehat{h}): \widehat{U}_{\ell} \rightarrow \widehat{U}_{\ell}$ is not the identity, while the restricted action $\Phi_{\ell+1}(\widehat{h}): \widehat{U}_{\ell+1} \rightarrow \widehat{U}_{\ell+1}$ is the identity.

By the definition of the conjugating map $\theta_{1}$ in Proposition 6.12 and more precisely the relation (44), the map $\theta_{1}\left(\Phi_{1}(\widehat{h})\right) \in \operatorname{Homeo}\left(\widehat{V}_{1}\right)$ restricts to $\Phi_{\ell}(\widehat{h})$ on $\widehat{U}_{\ell}$, and so is not the identity on $\widehat{U}_{\ell}$. Since $\widehat{U}_{\ell} \subset \widehat{V}_{\ell-1}$ by construction, this implies that $\theta_{1}\left(\Phi_{2}(\widehat{h})\right) \mid \widehat{V}_{\ell-1} \in \operatorname{Homeo}\left(\widehat{V}_{\ell-1}\right)$ is also not the identity, hence $\widehat{\tau}_{1}(\widehat{k}) \notin \bar{K}_{\ell-1}^{\prime}$.

Thus, if the chain $\mathcal{K}=\left\{K_{\ell} \mid \ell \geq 0\right\}$ is unbounded, then the chain $\overline{\mathcal{K}}^{\prime}=\left\{\bar{K}_{\ell}^{\prime} \mid \ell \geq 1\right\}$ is unbounded, and hence the action is $\left(V_{1}, H_{1}^{\prime}, \bar{\Psi}_{1}\right)$ is wild. This contradicts the choice of $V_{1}$, and completes the proof of Theorem 6.10.

REMARK 6.17. Here is a simple example that illustrates one of the main ideas in the above proof of Theorem 6.10 Let $\Phi: \mathbb{Z}^{n} \times \mathfrak{X} \rightarrow \mathfrak{X}$ be a faithful action, for $n \geq 2$. So $\left(\mathfrak{X}, \mathbb{Z}^{n}, \Phi\right)$ is an odometer action of the abelian group $\mathbb{Z}^{n}$ and is a free action. Let $\theta \in \mathbf{S L}(\mathbf{n}, \mathbb{Z})$ be a non-identity matrix, which acts via conjugation on $\mathbb{Z}^{n}$. Let $\Psi=\Phi^{\theta}$ be the action of $\mathbb{Z}^{n}$ on $\mathfrak{X}$ as defined by (6.3), which is again free. Then the identity map is a continuous orbit equivalence between $\left(\mathfrak{X}, \mathbb{Z}^{n}, \Phi\right)$ and $\left(\mathfrak{X}, \mathbb{Z}^{n}, \Psi\right)$. The map $\theta_{1}$ obtained in Proposition 6.12 is then just the restriction of $\theta$ to a subgroup of finite index in $\mathbb{Z}^{n}$. For a choice of group chains adapted to the actions, the map $\theta_{1}$ need not preserve the subgroups in these chains, but the map $\widehat{\tau}_{*}$ in (51) is the map on the inverse limit space $\mathfrak{X}$ induced by the action of $\Theta$. The proof above is a generalization of this example to the more general (and more complicated) non-abelian setting.

Finally, we give an application of Theorem 6.10 and the techniques used in its proof, to obtain an extension of Theorem 3.3 by Cortez and Medynets in [13], and Theorem 1.5 in [36].

THEOREM 6.18. Let $G$ and $G^{\prime}$ be finitely generated groups, and suppose that the Cantor action $\left(\mathfrak{X}^{\prime}, G^{\prime}, \Psi\right)$ is stable. Let $(\mathfrak{X}, G, \Phi)$ be a Cantor action which is continuously orbit equivalent to $\left(\mathfrak{X}^{\prime}, G^{\prime}, \Psi\right)$, then the actions are return equivalent.

Proof. By Theorem 6.10, the action $(\mathfrak{X}, G, \Phi)$ is stable. Thus, in the proof of Theorem 6.10, we can choose the clopen set $U_{1} \subset \mathfrak{X}$ so that the restricted action $\bar{\Phi}_{1}: H_{1} \times U_{1} \rightarrow U_{1}$ is topologically free.

Then in the proof of Lemma 6.14 the choice of $g=\beta(k, y) \in G$ such that $\Phi(g)(z)=\Psi(k)(z)$ is unique on $U_{1}$, so the map $\theta_{1}$ is injective. That is, the homomorphism $\theta_{1}: H_{1} \rightarrow H_{V_{1}}^{\prime}$ is an isomorphism onto its image. Set $H_{1}^{\prime \prime}=\theta_{1}\left(H_{1}\right) \subset H_{V_{1}}^{\prime}$.

Then (50) implies that the group chain $\mathcal{H}_{\mathcal{V}}^{\prime}=\left\{H_{1, \ell}^{\prime} \mid \ell \geq 1\right\}$ for the induced action $\left(V_{1}, H_{1}^{\prime}, \bar{\Psi}_{1}\right)$, is equivalent in the sense of Definition 3.5, to the group chain $\mathcal{H}^{\prime \prime}=\left\{H_{\ell}^{\prime \prime} \mid \ell \geq 1\right\}$ where $H_{1}^{\prime \prime}=H_{V_{1}}^{\prime}$, and $H_{\ell}^{\prime \prime}=\theta_{1}\left(H_{1, \ell}\right)$ for $\ell \geq 1$. Let $W_{1}^{\prime}=\widehat{\tau}_{*}\left(U_{1}\right) \subset V_{1}^{\prime}$ be the clopen subset corresponding to the truncated chain $\left\{H_{1, \ell}^{\prime} \mid \ell \geq 1\right\}$, where $\widehat{\tau}_{*}$ is the map in (51). Let $h_{1}: U_{1} \rightarrow W_{1}^{\prime}$ denote the map $\widehat{\tau}_{*}$ onto its image, which then conjugates the induced action $\left(U_{1}, H_{1}, \bar{\Phi}_{1}\right)$ with the Cantor action $\left(W_{1}^{\prime}, H_{\ell}^{\prime \prime}, \bar{\Psi}_{1}\right)$ which is induced from $\left(\mathfrak{X}^{\prime}, G^{\prime}, \Psi\right)$. Thus, the actions $\Phi$ and $\Psi$ are return equivalent. 


\section{Wild ACTIONS AND NON-HAUSDORFF ELEMENTS}

In this section, we consider Cantor actions $(\mathfrak{X}, G, \Phi)$ for which the stabilizer direct limit group $\Upsilon_{s}(\Phi)$ is unbounded, and derive some of their dynamical properties. Of special interest will be the existence of non-Hausdorff elements for the action, which are defined in Section 7.1

The notion of a non-Hausdorff element is local in $\mathfrak{X}$, and our first result is a generalization of Proposition 3.1 by Renault in [49. Proposition 7.2 below implies that a Cantor action with a nonHausdorff element has unbounded stabilizer group $\Upsilon_{s}(\Phi)$. Then in Theorem 7.5, we show that a Cantor action with a non-Hausdorff element must be dynamically wild. The converse implication, which is to give criteria for the existence of non-Hausdorff elements, is a much more subtle problem.

7.1. The germinal groupoid. The reduced $C^{*}$-algebra $C_{r}^{*}(\mathfrak{X}, G, \Phi)$ associated to a Cantor action $(\mathfrak{X}, G, \Phi)$ is an invariant of the continuous orbit equivalence class of the action. The study of the $K$-theory of $C_{r}^{*}(\mathfrak{X}, G, \Phi)$ offers another approach to the classification of Cantor actions, as used for example in the work [30]. This $C^{*}$-algebra can also be constructed using the germinal groupoid $\mathcal{G}(\mathfrak{X}, G, \Phi)$ associated to the action, as discussed for example by Renault in [47. Then one can ask how the properties of the $C^{*}$-algebra are related to the dynamical properties of the action, as discussed by Renault in [48.

In the work [9], Renault assumes that the Cantor action is topologically free, and thus the germinal groupoid $\mathcal{G}(\mathfrak{X}, G, \Phi)$ is a Hausdorff topological space, in order to avoid technical difficulties that arise otherwise. In fact, as remarked in Corollary 1.8 there are also wild actions for which $\mathcal{G}(\mathfrak{X}, G, \Phi)$ is still Hausdorff. Thus, the case where $\mathcal{G}(\mathfrak{X}, G, \Phi)$ has non-Hausdorff topology may be considered to be exceptional, and the fact that the topology is non-Hausdorff has implications for the algebraic structure of $C_{r}^{*}(\mathfrak{X}, G, \Phi)$, as discussed in [8, 24].

Recall first the definition of the germinal groupoid $\mathcal{G}(\mathfrak{X}, G, \Phi)$. For $g_{1}, g_{2} \in G$, we say that $\Phi\left(g_{1}\right)$ and $\Phi\left(g_{2}\right)$ are germinally equivalent at $x \in \mathfrak{X}$ if $\Phi\left(g_{1}\right)(x)=\Phi\left(g_{2}\right)(x)$, and there exists an open neighborhood $x \in U \subset \mathfrak{X}$ such that the restrictions agree, $\Phi\left(g_{1}\right)\left|U=\Phi\left(g_{2}\right)\right| U$. We then write $\Phi\left(g_{1}\right) \sim_{x} \Phi\left(g_{2}\right)$. For $g \in G$, denote the equivalence class of $\Phi(g)$ at $x$ by $[g]_{x}$. The collection of germs $\mathcal{G}(\mathfrak{X}, G, \Phi)=\left\{[g]_{x} \mid g \in G, x \in \mathfrak{X}\right\}$ is given the sheaf topology, and forms an étale groupoid modeled on $\mathfrak{X}$. We recall the following formulation of the Hausdorff property that was given by Winkelnkemper:

PROPOSITION 7.1. [52, Proposition 2.1] The germinal groupoid $\mathcal{G}(\mathfrak{X}, G, \Phi)$ is Hausdorff at $[g]_{x}$ if and only if, for all $\left[g^{\prime}\right]_{x} \in \mathcal{G}(\mathfrak{X}, G, \Phi)$ with $g \cdot x=g^{\prime} \cdot x$, if there exists a sequence $\left\{x_{i}\right\} \subset \mathfrak{X}$ which converges to $x$ such that $[g]_{x_{i}}=\left[g^{\prime}\right]_{x_{i}}$ for all $i$, then $[g]_{x}=\left[g^{\prime}\right]_{x}$.

Winkelnkemper showed in [52, Proposition 2.3] that for a smooth foliation $\mathcal{F}$ of a connected manifold $M$ for which the associated holonomy pseudogroup $\mathcal{G}_{\mathcal{F}}$ is generated by real analytic maps, then $\mathcal{G}_{\mathcal{F}}$ is a Hausdorff space. For Cantor actions, an analogous result holds for the LQA property.

PROPOSITION 7.2. If an action $(\mathfrak{X}, G, \Phi)$ is locally quasi-analytic, then $\mathcal{G}(\mathfrak{X}, G, \Phi)$ is Hausdorff.

Proof. Assume that $\mathcal{G}(\mathfrak{X}, G, \Phi)$ is not Hausdorff. Then there exists $g \in G$ and $x \in \mathfrak{X}$ such that $\mathcal{G}(\mathfrak{X}, G, \Phi)$ is non-Hausdorff at $[g]_{x}$. By Proposition 7.1 there exists $\left[g^{\prime}\right]_{x} \in \mathcal{G}(\mathfrak{X}, G, \Phi)$ with $g \cdot x=$ $g^{\prime} \cdot x$, and a sequence $\left\{x_{i}\right\} \subset \mathfrak{X}$ which converges to $x$ such that $[g]_{x_{i}}=\left[g^{\prime}\right]_{x_{i}}$ for all $i$, but $[g]_{x} \neq\left[g^{\prime}\right]_{x}$. Let $g^{\prime \prime}=g^{-1} g^{\prime} \in G$, then $g^{\prime \prime} \cdot x=x$ and $\left[g^{\prime \prime}\right]_{x_{i}}=[i d]_{x_{i}}$ for all $i$, but $\left[g^{\prime \prime}\right]_{x} \neq[i d]_{x}$.

The fact that the germ $\left[g^{\prime \prime}\right]_{x} \neq[i d]_{x}$ means that the action of $\Phi\left(g^{\prime \prime}\right)$ is not the identity in any open neighborhood of $x$. On the other hand, the germinal equalities $[g]_{x_{i}}=\left[g^{\prime}\right]_{x_{i}}$ for $i \geq 1$ imply there exists a sequence of open sets $x_{i} \in W_{i} \subset \mathfrak{X}$ for which the restriction of $\Phi\left(g^{\prime \prime}\right)$ to $W_{i}$ is the identity. Hence, there does not exists $\varepsilon>0$ such that $\Phi(g) \mid U$ is quasi-analytic for any open neighborhood $x \in U$ with diam $(U)<\varepsilon$. Thus, the action $(\mathfrak{X}, G, \Phi)$ is not locally quasi-analytic. 
7.2. Non-Hausdorff elements in the closure. Recall that by Theorem 5.3, a Cantor action $(\mathfrak{X}, G, \Phi)$ is a locally completely quasi-analytic (LCQA) action if and only if the stabilizer group $\Upsilon_{s}(\Phi)$ is bounded. Based on Proposition 7.1, we introduce the following notion:

DEFINITION 7.3. Let $(\mathfrak{X}, G, \Phi)$ be a Cantor action, and let $\mathfrak{G}(\Phi) \subset$ Homeo $(\mathfrak{X})$ denote the closure of the action. Then $\widehat{g} \in \mathfrak{G}(\Phi)$ is a non-Hausdorff element at $x \in \mathfrak{X}$ if:

(1) $\hat{g} \cdot x=x$; and there exists

(2) a sequence $\left\{x_{i} \mid i \geq 1\right\} \subset \mathfrak{X}$ converging to $x$; and

(3) clopen subsets $x_{i} \in W_{i} \subset \mathfrak{X}$;

such that for any clopen subset $x \in U \subset \mathfrak{X}$ the restriction of $\widehat{g}$ to $U$ is not the identity, while for all $i \geq 1$, we have $\widehat{g} \cdot W_{i}=W_{i}$ and the restriction of $\widehat{g}$ to $W_{i}$ is the identity.

We then have the following consequence of Proposition 7.2, which gives a connection between the unbounded property for $\Upsilon_{s}(\Phi)$ and the dynamics of the action.

COROLLARY 7.4. Let $(\mathfrak{X}, G, \Phi)$ be a Cantor action. Suppose that $\mathfrak{G}(\Phi)$ contains a non-Hausdorff element, then the stabilizer limit group $\Upsilon_{s}(\Phi)$ is unbounded. That is, the action is wild.

Proof. Assume that $\mathfrak{G}(\Phi)$ contains a non-Hausdorff element, then by Proposition 7.2 applied to the action of $\mathfrak{G}(\Phi)$, the action $(\mathfrak{X}, G, \Phi)$ is not locally completely quasi-analytic. Then by Theorem 5.3 $\Upsilon_{s}(\Phi)$ is unbounded.

7.3. Non-Hausdorff dynamics. We next give in Theorem 7.5 a sharper version of the conclusion of Corollary 7.4. As an application, Corollary 7.6 implies that the examples of wild actions constructed in [35, Section 9] cannot have non-Hausdorff elements in $\mathfrak{G}(\Phi)$. The arboreal constructions in Section 8 yield examples of Cantor actions with non-Hausdorff elements in $\Phi(G)$.

The difficulty in constructing non-Hausdorff elements can be seen from the following considerations. The hypothesis that $\Upsilon_{s}(\Phi)$ is unbounded implies that for $x \in \mathfrak{X}$, there exists a sequence of adapted clopen neighborhoods of $x$ and elements in $\mathfrak{G}(\Phi)_{x}$ which do not act as the identity on this open neighborhood, but do act as the identity on some smaller clopen neighborhoods. On the other hand, the non-Hausdorff property asserts there is some fixed element $\widehat{h} \in \mathfrak{G}(\Phi)_{x}$ so that for any adapted neighborhood with $x \in U$, the restricted action of $\Phi(\widehat{h})$ is not the identity on $U$, but the action is the identity on some clopen subset $V \subset U$. The distinction is that the non-Hausdorff condition in Proposition 7.1 is a statement about the local action of a fixed element $\widehat{h}$, while the wild hypothesis is a statement about the behavior of a sequence of elements in $\mathfrak{G}(\Phi)$. We use the interplay of these two notions in the proof of Theorem 7.5 .

Recall from Definition 4.10 that the limit group $\Upsilon_{s}(\Phi)$ for a Cantor action $(\mathfrak{X}, G, \Phi)$ has finite type if it is represented by an increasing chain $\mathcal{K}=\left\{K_{\ell} \mid \ell \geq 0\right\}$ where each $K_{\ell}$ is a finite group. Recall from Definition 4.18 that a Cantor action $(\mathfrak{X}, G, \Phi)$ is dynamically wild if the stabilizer group $\Upsilon_{s}(\Phi)$ is unbounded, and there is a proper inclusion $\Upsilon_{c}(\Phi) \subset \Upsilon_{s}(\Phi)$.

THEOREM 7.5. Let $(\mathfrak{X}, G, \Phi)$ be a Cantor action with a non-Hausdorff element $\widehat{h} \in \mathfrak{G}(\Phi)_{x}$, then $\Upsilon_{s}(\Phi)$ does not have finite type, and the action is dynamically wild.

Proof. Let $x \in \mathfrak{X}$, with $\widehat{g} \in \mathfrak{G}(\Phi)_{x}$ a non-Hausdorff element at $x$. Then by Definition [7.3, for any adapted clopen set $U$ with $x \in U$, the restriction of $\widehat{g}$ to $U$ is not the identity. In addition, there exists a sequence $\left\{x_{i} \mid i \geq 1\right\} \subset \mathfrak{X}$ of distinct points converging to $x$, with $\widehat{g} \cdot x_{i}=x_{i}$ for all $i \geq 1$, and clopen subsets $x_{i} \in W_{i} \subset \mathfrak{X}$ such that $\widehat{g} \cdot W_{i}=W_{i}$ and the restriction of $\widehat{g}$ to $W_{i}$ is the identity. It follows that $x \notin W_{i}$ for all $i \geq 1$.

Let $\mathcal{U}=\left\{U_{\ell} \subset \mathfrak{X} \mid \ell \geq 0\right\}$ be an adapted neighborhood basis for the action $\Phi$ at $x$.

Let $\mathcal{G}_{\mathcal{U}}^{x}=\left\{G_{\ell} \mid i \geq 0\right\}$ be the group chain associated to $\mathcal{U}$. Let $\left\{K_{\ell} \mid \ell \geq 0\right\}$ be its chain of stabilizer groups, and $\left\{Z_{\ell} \mid \ell \geq 0\right\}$ be its chain of centralizer groups as in Definition 4.14, 
Recall from Corollary 3.4 that the map $\widehat{\Theta}$ in Theorem 3.2 induces a homeomorphism of $G$-spaces $\Theta_{x}: \mathfrak{G}(\Phi) / \mathfrak{G}(\Phi)_{x} \cong \mathfrak{X} \rightarrow \widehat{G}_{\infty} / \mathcal{D}_{x} \cong X_{\infty}$.

For each $i \geq 1$, choose $\widehat{g}_{i} \in \widehat{G}_{\infty}$ such that $x_{i}=\widehat{g}_{i} \cdot x$.

As $x_{i} \in W_{i}$ and $x \notin W_{i}$, there exists $\ell_{i}>i$ such that $U_{\ell_{i}} \cap W_{i}=\emptyset$ and $\widehat{g}_{i} \cdot U_{\ell_{i}} \subset W_{i}$, hence $\widehat{g}_{i} \cdot U_{\ell_{i}} \cap U_{\ell_{i}}=\emptyset$. Moreover, as $x_{i}$ limits to $x$ there exists $j>i$ such that $x_{j} \in U_{\ell_{i}}$ and $x_{j} \notin W_{i}$.

As $\left\{U_{\ell} \mid \ell \geq 0\right\}$ is a neighborhood system about $x$, by passing to subsequences chosen recursively, we can assume that for $\ell \geq 1$ we have:

$$
U_{\ell} \cap W_{\ell}=\emptyset, x_{\ell}=\widehat{g}_{\ell} \cdot x, \widehat{g}_{\ell} \cdot U_{\ell} \subset W_{\ell}, x_{\ell+1} \notin W_{\ell} .
$$

Set $\widehat{h}_{\ell}=\widehat{g}_{\ell}^{-1} \widehat{g} \widehat{g}_{\ell}$. Then $\widehat{g} \cdot x_{\ell}=x_{\ell}$ implies that $\left(\widehat{g}_{\ell}^{-1} \widehat{g} \widehat{g}_{\ell}\right) \cdot x=x$, hence $\widehat{h}_{\ell} \cdot x=x$ so $\widehat{h}_{\ell} \in \mathcal{D}_{x}$.

Since $\widehat{g}$ acts as the identity on $W_{\ell}$ and $\widehat{g}_{\ell} \cdot U_{\ell} \subset W_{\ell}$, we obtain that $\widehat{h}_{\ell} \in K_{\ell}$.

Set $y_{\ell}=\widehat{g}_{\ell}^{-1} \cdot x$ and observe that $\widehat{h}_{\ell} \cdot y_{\ell}=y_{\ell}$ and that $y_{\ell} \notin U_{\ell+1}$ as $\widehat{g}_{\ell} \cdot U_{\ell+1} \cap U_{\ell+1}=\emptyset$.

We claim this implies that each $K_{\ell}$ is not a finite group, and hence $\Upsilon_{s}(\Phi)$ does not have finite type.

For $\ell \geq 0$, recall from (17) that $\widehat{G}_{\ell}=\widehat{C}_{\ell} \mathcal{D}_{x}$ is a clopen subset of $\widehat{G}_{\infty}$. Then $\Theta_{x}$ identifies the clopen set $U_{\ell} \subset \mathfrak{X}$ with $\widehat{G}_{\ell} / \mathcal{D}_{x} \subset \widehat{G}_{\infty}$. Thus, $\widehat{C}_{\ell}$ acts transitively on the quotient space

$$
\widehat{U}_{\ell}=\widehat{G}_{\ell} / \mathcal{D}_{x}=\left(\widehat{C}_{\ell} \mathcal{D}_{x}\right) / \mathcal{D}_{x}=\widehat{C}_{\ell} /\left(\widehat{C}_{\ell} \cap \mathcal{D}_{x}\right) .
$$

It is given that $\widehat{C}_{\ell} \subset \widehat{G}_{\ell}$ is a normal subgroup of $\widehat{G}_{\infty}$, hence for any $g \in G$ we have $g \widehat{C}_{\ell} g^{-1}=\widehat{C}_{\ell}$, thus $\widehat{C}_{\ell}$ also acts transitively on the clopen set $\widehat{U}_{\ell}^{g} \equiv g \cdot \widehat{U}_{\ell}$.

For each $\widehat{k} \in \widehat{C}_{\ell}$ define the conjugate element $\widehat{h}_{\ell}^{\widehat{k}}=\widehat{k} \widehat{h}_{\ell} \widehat{k}^{-1}$. Since $\widehat{h}_{\ell}$ acts as the identity on $U_{\ell}$, and $\widehat{k} \cdot U_{\ell}=U_{\ell}$ we have $\widehat{h}_{\ell}^{\widehat{k}} \in K_{\ell}$.

Finally, recall that $\widehat{g}$ is not the identity on any clopen neighborhood of $x$, and $\widehat{h}_{\ell}=\widehat{g}_{\ell}^{-1} \widehat{g} \widehat{g}_{\ell}$ satisfies $\widehat{h}_{\ell} \cdot y_{\ell}=y_{\ell}$ and is not the identity map in any open neighborhood of $y_{\ell}$. That is, the map $\widehat{h}_{\ell}$ has a non-trivial germ at $y_{\ell}$. Thus, each conjugate map $\widehat{h}_{\ell}^{\widehat{k}}$ has a fixed point at $\widehat{y}=\widehat{k} \cdot y_{\ell} \in U_{\ell}^{\widehat{g}_{\ell}^{-1}}$, and acts non-trivially on any neighborhood of $\widehat{y}$. Let $\mathcal{B}=\left\{\widehat{h}_{\ell} \mid \widehat{k} \in \widehat{C}_{\ell}\right\} \subset K_{\ell}$ be the collection of all such maps.

For $\phi \in \operatorname{Homeo}\left(\widehat{U}_{\ell}^{\widehat{g}_{\ell}^{-1}}\right)$ let $\operatorname{Fix}(\phi)=\left\{\widehat{y} \in \widehat{U}_{\ell}^{\widehat{g}_{\ell}^{-1}} \mid \phi(\widehat{y})=\widehat{y}\right\}$, and let $\operatorname{Germ}\left(\phi, \widehat{U}_{\ell}^{\widehat{g}_{\ell}^{-1}}\right)$ denote the subspace of $\operatorname{Fix}(\phi)$ such that $\phi$ has non-trivial germ at $\widehat{y}$. If $\widehat{y} \in W$ is a clopen subset of $\operatorname{Germ}\left(\phi, \widehat{U}_{\ell}^{\widehat{g}_{\ell}^{-1}}\right)$, then $W \subset \operatorname{Fix}(\phi)$, and then the germ of $\phi$ is trivial at $\widehat{y}$. This is a contradiction, so the set $\operatorname{Germ}\left(\phi, \widehat{U}_{\ell}^{\widehat{g}_{\ell}^{-1}}\right)$ has no interior.

Now, suppose that the collection $\mathcal{B}$ is countable. Then the set of points $\widehat{y} \in \widehat{U}_{\ell}^{\widehat{g}_{\ell}^{-1}}$ for which there exists some $\phi \in \mathcal{B}$ for which $\widehat{y}$ is a fixed point with non-trivial holonomy is a countable union of subsets of $\widehat{U}_{\ell}^{\widehat{g}_{\ell}^{-1}}$ without interior. Since $\widehat{U}_{\ell}^{\widehat{g}_{\ell}^{-1}}$ is a Cantor space hence is Baire, by the Baire Category Theorem, this union has no interior. This contradicts the previous observation that every point of $\widehat{U}_{\ell}^{\widehat{g}_{\ell}^{-1}}$ is a fixed point for some $\phi \in \mathcal{B}$ with non-trivial germ. Thus, $\mathcal{B}$ must be an uncountable collection. In particular, $K_{\ell}$ is an uncountable group for all $\ell \geq 0$.

Note that the above Baire argument is based on the same ideas as in the proofs of Theorem 1 by Epstein, Millet and Tischler in [23], and Theorem 3.6 by Renault in [49].

Next, we show that the action $(\mathfrak{X}, G, \Phi)$ is dynamically wild. Suppose not, then we have $\Upsilon_{c}(\Phi)=$ $\Upsilon_{s}(\Phi)$ which implies that there exists an increasing subsequence $\left\{\ell_{i} \mid i \geq 1\right\}$ such that the inclusion $Z_{\ell_{i}} \subset K_{\ell_{i}}$ is an isomorphism for all $i \geq 1$. Then for each $i \geq 1$ we have the collection of maps $\left\{\widehat{h}_{\ell_{i}} \mid \widehat{k} \in \widehat{C}_{\ell_{i}}\right\} \subset Z_{\ell_{i}}$. For simplicity, set $\ell_{i}=\ell$. 
Recall that by construction for $\ell \geq 1$ the maps $h_{\ell}$ have non-trivial germinal holonomy at $y_{\ell}$. Moreover, since $\widehat{g}_{\ell}^{-1}$ conjugates $y_{\ell}$ to $x, \widehat{h}_{\ell}$ is non-Hausdorff at $y_{\ell}$. Therefore, there is a clopen subset $W^{\prime} \subset \widehat{U}_{\ell}^{\widehat{g}_{\ell}^{-1}}$ such that $\widehat{h}_{\ell}$ is the identity on $W^{\prime}$.

Since $\widehat{C}_{\ell}$ preserves and acts transitively on $\widehat{U}_{\ell}^{\widehat{g}_{\ell}^{-1}}$, there is $\widehat{k} \in \widehat{C}_{\ell}$ such that $\widehat{k} \cdot y_{\ell} \in W^{\prime}$. Then the element $\widehat{h}_{\ell}^{\widehat{k}}$ fixes an open neighborhood of $y_{\ell}$. But this contradicts the fact that $\widehat{h}_{\ell}^{\widehat{k}}=\widehat{k}_{h_{\ell}} \widehat{k}^{-1}=\widehat{h}_{\ell}$ is not the identity map on any clopen neighborhood of the fixed point $y_{\ell}$. Thus, $Z_{\ell}=K_{\ell}$ is impossible.

COROLLARY 7.6. Let $(\mathfrak{X}, G, \Phi)$ be a Cantor action with discriminant group $\mathcal{D}_{x}$ at $x \in \mathfrak{X}$. Suppose that for each $\widehat{g} \in \mathcal{D}_{x}$, the intersection $\mathcal{D}_{x} \cap\left\{\widehat{h}^{-1} \widehat{g} \widehat{h} \mid \widehat{h} \in \widehat{G}_{\infty}\right\}$ is finite, then the germinal groupoid of the action is Hausdorff.

Proof. Suppose that $\widehat{g} \in \mathcal{D}_{x}$ is a non-Hausdorff element. Then the proof of Theorem 7.5 shows that the the set of conjugacy classes of $\widehat{g}$ in $\mathcal{D}_{x}$ is an infinite set.

It is a basic question to find a converse to the conclusion of Theorem 7.5 . That is, to find sufficient conditions so that a Cantor action which is dynamically wild must have a non-Hausdorff element.

In the next Section 8, examples of wild Cantor actions are constructed using the "automata" method, which is a well-known technique in Geometric Group Theory, and defines a homeomorphism using a recursive definition along the branches of a tree. It would be very interesting to understand if the use of automata is the only approach to constructing actions with non-Hausdorff elements, or whether there are possibly alternative general methods for their construction.

\section{EXAMPLES OF TREE AUTOMORPHISMS}

In this section, we present examples constructed as actions on the boundary of an infinite binary tree to illustrate some of the properties of wild Cantor actions. The boundary of a tree $T$ is identified with the set of all infinite paths in the tree, and can also be viewed as a collection of infinite sequences of 0's and 1's. It is a Cantor set by a standard argument. To construct our examples, we use an approach well-known in Geometric Group Theory, of defining a homeomorphism of the Cantor set at the boundary recursively, by specifying how it acts at each level of the tree. For example, the Grigorchuk group and the Basilica group are usually defined this way; see [33, 44, and other works.

8.1. Actions on trees. We start by explaining the notation and the method for the recursive construction of a homeomorphism of the boundary of a tree.

Let $T$ be a binary tree, that is, $T$ consists of the vertex set $V=\bigsqcup_{n \geq 0} V_{n}$ and of the set of edges $E$ with the following properties: For $n \geq 0$

(1) $\operatorname{card}\left(V_{n}\right)=2^{n}$.

(2) For each $v \in V_{n}$, there are exactly two vertices in $V_{n+1}$ joined to $v$ by edges.

(3) For each $v \in V_{n}$, with $n>0$, there is exactly one vertex in $V_{n-1}$ joined to $v$ by an edge.

We denote by $\mathcal{P}(T)$ the set of all infinite connected paths in $T$.

It is sometimes convenient to label the vertices in $V$ by 0 's and 1 's as follows. The single vertex in $V_{0}$ is not labelled; the two vertices in $V_{1}$ are labelled one by 0 , and another one by 1 . Now suppose the vertices in $V_{n}$ are labelled by words of length $n$ consisting of 0 's and 1's. Since there are $2^{n}$ such distinct words, we can assign to each vertex in $V_{n}$ a unique word, and to every word of length $n$ we can assign a vertex. Proceed to label the vertices in $V_{n+1}$ as follows. Let $v \in V_{n}$ be labelled by a word $s_{1} s_{2} \cdots s_{n}$. Since $T$ is binary, there are two vertices, $w_{1}$ and $w_{2}$ in $V_{n+1}$ which are joined to $v$ by edges. Label $w_{1}$ by $s_{1} s_{2} \cdots s_{n} 0$ and $w_{2}$ by $s_{1} s_{2} \cdots s_{n} 1$. 
It follows that every infinite sequence $s_{1} s_{2} \cdots$, where $s_{i} \in\{0,1\}$ for $i \geq 1$, corresponds to a unique path $s$ in the tree $T$. Namely, $s$ passes through the vertex labelled by $s_{1}$ in $V_{1}$, by $s_{1} s_{2}$ in $V_{2}$ and, inductively, $s$ passes through the vertex labelled by $s_{1} s_{2} \cdots s_{n}$ in $V_{n}$ for $n \geq 1$.

Next, let $w=w_{1} w_{2} \cdots w_{n}$ be a word of length $n$ in 0 's and 1's. We denote by $w T$ the subtree of $T$ which contains all infinite paths which start with $w$, that is, the path space of $w T$ is given by

$$
\mathcal{P}(w T)=\left\{s_{1} s_{2} \cdots s_{m} \cdots \in \mathcal{P}(T) \mid s_{i}=w_{i} \text { for } 1 \leq i \leq n\right\} .
$$

Clearly every path in $\mathcal{P}(w T)$ contains the vertex in $V_{n}$ labelled by $w$. The set $\mathcal{P}(w T)$ is a clopen subset of $\mathcal{P}(T)$. There is an obvious homeomorphism

$$
\psi_{w}: \mathcal{P}(w T) \rightarrow \mathcal{P}(T): w_{1} w_{2} \cdots w_{n} s_{n+1} s_{n+2} \cdots \mapsto s_{n+1} s_{n+2} \cdots,
$$

which induces a homeomorphism between $w T$ and $T$ which preserves the paths. Also, we can write $T=v_{0} \cup 0 T \cup 1 T$, where $v_{0}$ is the unique vertex in $V_{0}$.

We now explain the recursive definition of homeomorphisms of $\mathcal{P}(T)$. Let $\sigma$ be the non-trivial permutation of a set of two elements, that is, if this set is $\{0,1\}$, then $\sigma$ interchanges 0 and 1 . Denote by $\operatorname{Aut}(T)$ the automorphism group of the tree $T$, that is, an element $h \in \operatorname{Aut}(T)$ is a homeomorphism of $\mathcal{P}(T)$ which for all $n \geq 1$ restricts to a permutation of $V_{n}$. Let $h, g \in \operatorname{Aut}(T)$, then define an automorphism $(h, g)$ of $T$ by declaring that it restricts to the trivial permutation of $V_{1}$, and for every $s=s_{1} s_{2} \cdots \subset 0 T$ one has

$$
(h, g)(s)=\psi_{0}^{-1} \circ h \circ \psi_{0}(s),
$$

and for every $s \subset 1 T$ one has

$$
(h, g)(s)=\psi_{1}^{-1} \circ g \circ \psi_{1}(s) .
$$

That is, the automorphism $h$ is applied to the branch of the tree through the first vertex $\{0\}$ of $V_{1}$, and $g$ is applied to the branch of the tree through the second vertex $\{1\}$ of $V_{1}$. We compose the maps on the left.

We now give an example of a recursive definition of a homeomorphism of $T$. Define $a$ such that

$$
a=(a, 1) \sigma,
$$

where 1 denotes the identity in $\operatorname{Aut}(T)$. Then for every $s=s_{1} s_{2} \cdots$ the map $\sigma$ maps $s_{1}$ to $\left(s_{1}+1\right)$ mod 2 and fixes the rest of the sequence, so we can write, with a slight abuse of notation,

$$
\sigma(s)=\sigma\left(s_{1}\right) s_{2} s_{3} \cdots .
$$

Next, we apply $(a, 1)$ to $\sigma(s)$. If $\sigma\left(s_{1}\right)=1$, then by the rule (54), the element $a$ acts as the identity map on $\sigma\left(s_{1}\right) T=1 T$. If $\sigma\left(s_{1}\right)=0$, then by (53)

$$
a(s)=\sigma\left(s_{1}\right) a\left(s_{2} s_{3} \cdots\right)=0 a\left(s_{2} s_{3} \cdots\right),
$$

so we need to compute $a\left(s_{2} s_{3} \cdots\right)$. We compute that $\sigma\left(s_{2}\right)=\left(s_{2}+1\right) \bmod 2$, and then we have to apply $a$ to $\sigma\left(s_{1}\right) \sigma\left(s_{2}\right) s_{3} \cdots=0 \sigma\left(s_{2}\right) s_{3} \cdots$, that is, the image of $s$ is computed recursively. For example,

$$
a\left(0001110^{\infty}\right)=1001110^{\infty}, \quad a\left(11001^{\infty}\right)=00101^{\infty}, \quad a\left(1^{\infty}\right)=0^{\infty},
$$

where $1^{\infty}$ denotes an infinite sequence of 1 's, and $0^{\infty}$ denotes an infinite sequence of 0 's.

The element $a$, defined by (55), generates a group isomorphic to the integers $\mathbb{Z}$, which acts freely and transitively on every vertex set $V_{n}, n \geq 1$. Such an arboreal action is often called an odometer, a standard odometer or an adding machine in the literature [44, 46. In this paper, we use the term "odometer" in a more general sense; that is, an odometer is an equicontinuous minimal action of any group $G$ on a Cantor set $\mathfrak{X}$. To distinguish between the two notions, we use the term cyclic odometer for the action generated by a single element $a$ as described above. 
8.2. Rigidity of Cantor actions. In this section we illustrate the discussion in Section 6.2 of various definitions of rigidity. We present an example of orbit equivalent actions, where one of the actions is free, and another one is stable but not topologically free. This example motivates the introduction of the notion of return equivalence in Section 6.2.

Let $T$ be a binary tree with the Cantor set boundary $\mathcal{P}(T)$, and set $\mathfrak{X}=\mathcal{P}(T)$. Let $a_{1}=\left(a_{1}, 1\right) \sigma$ be the cyclic odometer defined in (55). Let $a_{2}=\left(a_{1}, 1\right)$, that is, $a_{2}$ acts as a cyclic odometer on the clopen set $\mathcal{P}(0 T)$, and as the identity map on $\mathcal{P}(1 T)$. We also note that $a_{2}\left|\mathcal{P}(0 T)=a_{1}^{2}\right| \mathcal{P}(0 T)$.

Define $H=\left\langle a_{1}\right\rangle$ and $G=\left\langle a_{1}, a_{2}\right\rangle$, so we have the actions $(\mathfrak{X}, G)$ and $(\mathfrak{X}, H)$, where we omit $\Phi$ and $\Psi$ from the notation since $G$ and $H$ are already defined as subgroups of Homeo( $\mathfrak{X})$. Both actions are minimal, since the orbit of any path $\left(v_{n}\right)_{n \geq 1} \in \mathcal{P}(T)$ under the powers of $a_{1}$ is dense in $\mathfrak{X}$. It is easy to see that the group $G$ is non-commutative.

The action $(\mathfrak{X}, G)$ is not topologically free, as $a_{2}$ acts as the identity on the clopen set $\mathcal{P}(1 T)$. On the other hand, $(\mathfrak{X}, H)$ is a free action of a cyclic odometer.

We now show the actions $(\mathfrak{X}, G)$ and $(\mathfrak{X}, H)$ are continuously orbit equivalent. Let $h: \mathfrak{X} \rightarrow \mathfrak{X}$ be the identity map, then we define the maps $\alpha: \mathfrak{X} \times G \rightarrow H$ and $\beta: \mathfrak{X} \times H \rightarrow G$ (as in Section 6.1) as follows.

Since $a_{2}$ acts on $0 T$ as $a_{1}^{2}$ and on $1 T$ as the identity map, for any $x \in \mathfrak{X}$ the orbits of the action of $G$ and $H$ coincide as sets. Since the action of $H$ is free, given $x \in \mathfrak{X}$ and $g \in G$ there exists a unique element $h \in H$ such that $g \cdot x=h \cdot x$. We define $\alpha(g, x)=h$. We show that $\alpha$ is constant when restricted to $\mathcal{P}(0 T)$, and to $\mathcal{P}(1 T)$.

So let $\mathcal{U}=\{\mathcal{P}(0 T), \mathcal{P}(1 T)\}$ be a partition of $\mathcal{P}(T)$. Any element $g \in G$ can be written as a finite word $g=a_{1}^{k_{n}} a_{2}^{k_{n-1}} \cdots a_{1}^{k_{2}} a_{2}^{k_{1}}$, with $k_{j} \in \mathbb{Z}$ for $1 \leq j \leq n$. The actions of any power of $a_{2}$ and of even powers of $a_{1}$ preserve $\mathcal{P}(w T)$, and the actions of odd powers of $a_{1}$ interchange $\mathcal{P}(0 T)$ and $\mathcal{P}(1 T)$. It follows that $x$ and $y$ are in the same set of the partition $\mathcal{U}$ if and only if $a_{2}^{k_{1}} \cdot x$ and $a_{2}^{k_{1}}(y)$ are in the same set of $\mathcal{U}$. Implementing induction on the number of elements in the word decomposition of $g$, we obtain that $x$ and $y$ are in the same set of $\mathcal{U}$ if and only if $g \cdot x$ and $g \cdot y$ are in the same set of $\mathcal{U}$.

Define the map $\beta: H \times \mathfrak{X} \rightarrow G$ by $\beta\left(a_{1}^{k}, x\right)=a_{1}^{k}$. The map $\beta$ is constant on $\mathfrak{X}$, so is continuous. Thus for $h=i d, \alpha$ and $\beta$ yield a continuous orbit equivalence between the actions $(\mathfrak{X}, G)$ and $(\mathfrak{X}, H)$. By Theorem 1.4 the action $(\mathfrak{X}, G)$ is stable.

We also note that the map $\alpha_{x}: G \rightarrow H$ obtained by specializing $\alpha_{x}=\alpha(-, x)$ for $x \in \mathcal{P}(T)$ is not injective. For example, for $x \in \mathcal{P}(T)$ we have

$$
\alpha_{x}^{-1}\left(a_{1}^{2}\right) \supset\left\{a_{1}^{2}, a_{2}\right\} .
$$

Note that this example can be considered as an explicit form of the construction in [36, Example A.4].

8.3. Dynamically wild actions. Let $T$ be a binary tree with the Cantor set boundary $\mathcal{P}(T)$, we set $\mathfrak{X}=\mathcal{P}(T)$. Let $r \geq 3,2 \leq s \leq r$, and consider a group $G$ generated by the homeomorphisms

$$
a_{1}=\sigma, a_{s+1}=\left(a_{s}, a_{r}\right), a_{i}=\left(a_{i-1}, 1\right) \text { for } 3 \leq i \leq s, s+1 \leq i \leq r .
$$

Groups generated by the recursive rules (56) arise as iterated monodromy groups of quadratic post-critically finite polynomials 44, 4. They have been extensively studied, for example, in [4, and also in other works. For example, in [46 it was shown that the closure of the action of an iterated monodromy group, associated to a post-critically finite quadratic polynomial with strictly pre-periodic post-critical orbit of length $r$, where the periodic part has length $r-1$, is conjugate to the closure of the action of the group $G$ generated by (56).

Let $G$ be a group generated by (56), and let $\mathfrak{G}(\Phi)$ be the closure of the action. It was shown in 41, Theorem 1.3] (see also [40]) that $G$ contains non-Hausdorff elements, namely the generators $a_{s+1}, \ldots, a_{r}$ are non-Hausdorff. Then by Theorem 7.5 the action of the group $G$ is dynamically wild; that is, there is a proper inclusion of the direct limit groups $\Upsilon_{c}(\Phi) \subset \Upsilon_{s}(\Phi)$. 
8.4. Infinite stabilizer group. Theorem 7.5 shows that if a group $G$, or more generally its closure $\mathfrak{G}(\Phi)$ has a non-Hausdorff element, then the groups $K_{0} \subset K_{1} \subset K_{2} \subset \cdots \mathcal{D}_{x}$ in the stabilizer chain $\mathcal{K}(\mathcal{U})$ must be infinite. In specific examples it may be difficult to compute the groups $K_{\ell}, \ell \geq 1$, explicitly. We now give an example of an action for which this can be done.

Let $T$ be a binary tree with the Cantor set boundary $\mathcal{P}(T)$, we set $\mathfrak{X}=\mathcal{P}(T)$, and let $G$ be generated by (56) with $r=2 s$ and $s \geq 2$.

By definition $a_{2}, \ldots, a_{s}, a_{s+2}, \ldots, a_{2 s}$ are the identity on the clopen set $\mathcal{P}(1 T)$ of all sequences starting with digit 1 . Since $a_{s+1}=\left(a_{s}, a_{2 s}\right)$, and $a_{2 s}$ is the identity on $\mathcal{P}(1 T), a_{s+1}$ is the identity on the set $\mathcal{P}(11 T)$ of all sequences starting with a finite word 11.

Choose a path $x \in \mathcal{P}(11 T)$, that is, $x=11 x_{3} x_{4} \ldots$, and for $\ell \geq 1$ let $U_{\ell}$ be a clopen neighborhood

$$
U_{\ell}=\left\{s_{1} s_{2} s_{3} \ldots \mid x_{i}=s_{i} \text { for } 1 \leq i \leq \ell\right\} .
$$

Then every $U_{\ell} \subset \mathcal{P}(11 T)$, and so the generators $a_{2}, \ldots, a_{2 s}$ act trivially on $U_{\ell}$, in other words,

$$
\left\langle a_{2}, \ldots, a_{s}, a_{s+1}, \ldots, a_{2 s}\right\rangle \subset K_{\ell} .
$$

Consider the compositions $a_{i} a_{j} \in K_{\ell}$, with $i<j$. By [46, Proposition 3.1.9] if $j=i+s$, then $a_{i} a_{j}$ has infinite order. In particular, $a_{s} a_{2 s}$ has infinite order. It follows that $G \cap K_{\ell}$ is infinite, and so $K_{\ell}$ is a Cantor group.

\section{REFERENCES}

[1] J. Álvarez López and A. Candel, Equicontinuous foliated spaces, Math. Z., 263:725-774, 2009.

[2] J. Álvarez López and M. Moreira Galicia, Topological Molino's theory, Pacific. J. Math., 280:257-314, 2016.

[3] J. Auslander, Minimal flows and their extensions, North-Holland Mathematics Studies, Vol. 153, NorthHolland Publishing Co., Amsterdam, 1988.

[4] L. Bartholdi and V. Nekrashevych Iterated monodromy groups of quadratic polynomials, I, Groups Geom. Dyn., 2:309-336, 2008.

[5] S. Bezuglyi and K. Medynets, Full groups, flip conjugacy, and orbit equivalence of Cantor minimal systems, Colloq. Math., 110:409-429, 2008.

[6] M. Boyle, Topological orbit equivalence and factor maps in symbolic dynamics, Ph.D. Thesis, University of Washington, 1983.

[7] M. Boyle and J. Tomiyama, Bounded topological orbit equivalence and $C^{*}$-algebras, J. Math. Soc. Japan, 50:317-329, 1998.

[8] J. Brown, L. Clark, L. Orloff and C. Farthing, Simplicity of algebras associated to étale groupoids, Semigroup Forum, 88:433-452, 2014.

[9] A. Clark and S. Hurder, Homogeneous matchbox manifolds, Trans. A.M.S., 365:3151-3191, 2013.

[10] A. Clark, S. Hurder and O. Lukina, Classifying matchbox manifolds, Geom \& Top, 23:1-38, 2019; arXiv:1311.0226.

[11] A. Connes, J. Feldman and B. Weiss, An amenable equivalence relation is generated by a single transformation, Ergodic Theory Dynamical Systems, 1:431-450, 1981.

[12] M.-I. Cortez and S. Petite, G-odometers and their almost one-to-one extensions, J. London Math. Soc., $78(2): 1-20,2008$.

[13] M.I. Cortez and K. Medynets, Orbit equivalence rigidity of equicontinuous systems, J. Lond. Math. Soc. (2), 94:545-556, 2016

[14] M.I. Cortez and S. Petite, On the centralizers of minimal aperiodic actions on the Cantor set, preprint; arXiv:1807.04654.

[15] Y. de Cornulier, Groupes pleins-topologiques (d'après Matui, Juschenko, Monod, ...), Astérisque, 361:183-223, 2014.

[16] T. Downarowicz, Survey of odometers and Toeplitz flows, in Algebraic and topological dynamics, Contemp. Math., Vol. 385: 7-37, Amer. Math. Soc., Providence, RI, 2005.

[17] J. Dyer, S. Hurder and O. Lukina, The discriminant invariant of Cantor group actions, Topology Appl., 208: 64-92, 2016.

[18] J. Dyer, S. Hurder and O. Lukina, Growth and homogeneity of matchbox manifolds, Indag. Math., 28:145-169, 2017.

[19] J. Dyer, S. Hurder and O. Lukina, Molino theory for matchbox manifolds, Pacific J. Math., 289:91-151, 2017.

[20] S. Eilenberg and N. Steenrod, Foundations of algebraic topology, Princeton University Press, Princeton, New Jersey, 1952.

[21] R. Ellis, A semigroup associated with a transformation group, Trans. Amer. Math. Soc., 94:272-281, 1969.

[22] R. Ellis and W.H. Gottschalk, Homomorphisms of transformation groups, Trans. Amer. Math. Soc., 94:258$271,1969$. 
[23] D.B.A. Epstein, K.C. Millet, and D. Tischler, Leaves without holonomy, Jour. London Math. Soc., 16:548-552, 1977.

[24] R. Exel, Non-Hausdorff étale groupoids, Proc. Amer. Math. Soc., 139:897-907, 2011.

[25] R. Fokkink and L. Oversteegen, Homogeneous weak solenoids, Trans. Amer. Math. Soc., 354(9):3743-3755, 2002.

[26] A. Furman, Orbit equivalence rigidity, Ann. of Math. (2), 150:1083-1108, 1999.

[27] D. Gaboriau, Orbit equivalence and measured group theory, Proceedings of the International Congress of Mathematicians. Volume III, Hindustan Book Agency, New Delhi, 2010, pages 1501-1527.

[28] T. Giordano, I. Putman and C. Skau, Full groups of Cantor minimal systems, Israel J. Math., 111:285-320, 1999.

[29] T. Giordano, H. Matui, I. Putnam, and C. Skau, Orbit equivalence for Cantor minimal $\mathbb{Z}^{d}$-systems, Invent. Math., 179: 119-158, 2010.

[30] T. Giordano, I. Putman and C. Skau, $\mathbb{Z}^{d}$-odometers and cohomology, Groups Geom. Dyn., 13:909-938, 2019.

[31] E. Glasner and B. Weiss, Weak orbit equivalence of Cantor minimal systems, Internat. J. Math., 6:559-579, 1995.

[32] E. Glasner, Enveloping semigroups in topological dynamics, Topology Appl., 154:2344-2363, 2007.

[33] R.I. Grigorchuk, Degrees of growth of finitely generated groups and the theory of invariant means, Izv. Akad. Nauk SSSR Ser. Mat., 48:939-985, 1984.

[34] A. Haefliger, Pseudogroups of local isometries, in Differential Geometry (Santiago de Compostela, 1984), edited by L.A. Cordero, Res. Notes in Math., 131:174-197, Boston, 1985.

[35] S. Hurder and O. Lukina, Wild solenoids, Transactions A.M.S., 371:4493-4533, 2019.

[36] S. Hurder and O. Lukina, Orbit equivalence and classification of weak solenoids, Indiana Univ. Math. J., to appear; arXiv:1803.02098.

[37] S. Kobayashi and K. Nomizu, Foundations of differential geometry. Vol. II, Interscience Tracts in Pure and Applied Mathematics, No. 15 Vol. II , Interscience Publishers John Wiley \& Sons, Inc., New York-London-Sydney, 1969.

[38] X. Li, Continuous orbit equivalence rigidity, Ergodic Theory Dynam. Systems, 38:1543-1563, 2018.

[39] A. Lubotzky, Torsion in profinite completions of torsion-free groups, Quart. J. Math. Oxford Ser. (2) 44:327-332, 1993

[40] O. Lukina, Arboreal Cantor actions, J. Lond. Math. Soc. (2), 99:678-706, 2019.

[41] O. Lukina, Galois groups and Cantor actions, submitted; arXiv:1809.08475.

[42] C. McCord, Inverse limit sequences with covering maps, Trans. A.M.S., 114:197-209, 1965.

[43] J. Munkres, Elements of algebraic topology, Addison-Wesley Publishing Company, Menlo Park, CA, 1984.

[44] V. Nekrashevych, Self-similar groups, Mathematical Surveys and Monographs, Vol. 117, American Mathematical Society, Providence, RI, 2005.

[45] V. Nekrashevych, Palindromic subshifts and simple periodic groups of intermediate growth, Ann. of Math. (2), 187:667-719, 2018.

[46] R. Pink, Profinite iterated monodromy groups arising from quadratic polynomials; preprint; arXiv: 1307.5678.

[47] J. Renault, A groupoid approach to $C^{*}$-algebras, Lecture Notes in Math., vol. 793, 1980.

[48] J. Renault, Transverse properties of dynamical systems, in Representation theory, dynamical systems, and asymptotic combinatorics, Amer. Math. Soc. Transl. Ser. 2, Vol. 217, Amer. Math. Soc., Providence, RI, 2006, pages 185-199.

[49] J. Renault, Cartan subalgebras in $C^{*}$-algebras, Irish Math. Soc. Bull., 61:29-63, 2008.

[50] J.T. Rogers, Jr. and J.L. Tollefson, Homogeneous inverse limit spaces with non-regular covering maps as bonding maps, Proc. Amer. Math. Soc. 29: 417-420, 1971.

[51] R. Schori, Inverse limits and homogeneity, Trans. A.M.S., 124:533-539, 1966

[52] E. Winkelnkemper, The graph of a foliation, Ann. Global Ann. Geo., 1:51-75, 1983.

Steven Hurder, Department of Mathematics, University of Illinois at Chicago, 322 SeO (m/c 249 ), $851 \mathrm{~S}$ Morgan Street, Chicago, IL 60607-7045

E-mail address: hurder@uic.edu

Olga Lukina, Faculty of Mathematics, University of Vienna, Oskar-Morgenstern-Platz 1, 1090 Vienna, Austria

E-mail address: olga.lukina@uivie.ac.at 